\title{
The component structure of conformal supergravity invariants in six dimensions
}

\author{
Daniel Butter, $^{a, b}$ Joseph Novak ${ }^{c}$ and Gabriele Tartaglino-Mazzucchelli ${ }^{d}$ \\ ${ }^{a}$ Nikhef Theory Group, \\ Science Park 105, 1098 XG Amsterdam, The Netherlands \\ ${ }^{b}$ George and Cynthia Woods Mitchell Institute for Fundamental Physics and Astronomy, \\ Texas A $6 M$ University, \\ College Station, TX 7r843, U.S.A. \\ ${ }^{c}$ Max-Planck-Institut für Gravitationsphysik, Albert-Einstein-Institut, \\ Am Mühlenberg 1, D-14476 Golm, Germany \\ ${ }^{d}$ Instituut voor Theoretische Fysica, KU Leuven, \\ Celestijnenlaan 200D, B-3001 Leuven, Belgium \\ E-mail: dbutter@tamu.edu, joseph.novak@aei.mpg.de, \\ Gabriele.Tartaglino-Mazzucchelli@fys.kuleuven.be
}

ABSTRACT: In the recent paper arXiv:1606.02921, the two invariant actions for 6D $\mathcal{N}=(1,0)$ conformal supergravity were constructed in superspace, corresponding to the supersymmetrization of $C^{3}$ and $C \square C$. In this paper, we provide the translation from superspace to the component formulation of superconformal tensor calculus, and we give the full component actions of these two invariants. As a second application, we build the component form for the supersymmetric $F \square F$ action coupled to conformal supergravity. Exploiting the fact that the $\mathcal{N}=(2,0)$ Weyl multiplet has a consistent truncation to $\mathcal{N}=(1,0)$, we then verify that there is indeed only a single $\mathcal{N}=(2,0)$ conformal supergravity invariant and reconstruct most of its bosonic terms by uplifting a certain linear combination of $\mathcal{N}=(1,0)$ invariants.

KEYwords: Conformal Field Theory, Supergravity Models, Superspaces

ARXIV EPRINT: 1701.08163 


\section{Contents}

1 Introduction 1

2 The $6 \mathrm{D} \mathcal{N}=(1,0)$ Weyl multiplet from superspace $\quad 4$

2.1 Component fields and curvatures from superspace 5

2.2 Analysis of the curvature constraints 8

$\begin{array}{lll}2.3 & \text { Different choices of conventional constraints } & 10\end{array}$

$\begin{array}{ll}2.4 \text { The supersymmetry transformations } & 14\end{array}$

3 The supersymmetric $C^{\mathbf{3}}$ invariant $\quad 14$

$\begin{array}{lll}3.1 & \text { The } A \text { action principle } & 15\end{array}$

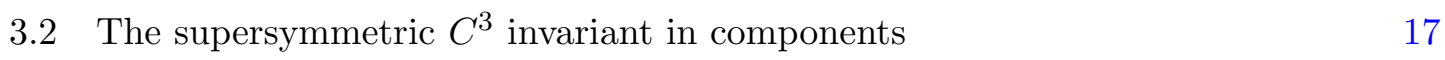

4 The supersymmetric $C \square C$ invariant $\quad 18$

$\begin{array}{ll}4.1 \text { The } B \text { action principle } & 19\end{array}$

4.2 The supersymmetric $C \square C$ invariant in components 21

4.3 The supersymmetric $F \square F$ invariant in components 23

5 The $\mathcal{N}=(2,0)$ conformal supergravity invariant $\quad 25$

6 Discussion $\quad 28$

A $6 \mathrm{D} \mathcal{N}=(1,0)$ conformal superspace $\quad 31$

A.1 The superconformal algebra 31

A.2 Gauging the superconformal algebra in conformal superspace 32

A.3 Different superspace frames 34

$\begin{array}{lr}\text { B Relating notation and conventions } & 39\end{array}$

\section{Introduction}

The invariants for conformal gravity naturally arise in the study of conformal field theories on curved manifolds. Deser and Schwimmer divided the possible conformal anomalies into two families, type A and type B [1]. Type A anomalies are topological and always involve the Euler term, while type B anomalies are Weyl invariants built (in the purely gravitational case) from the Riemann tensor and its derivatives. In six dimensions, there are three independent conformal gravity invariants parametrizing the type B anomalies. 
Their Lagrangians are

$$
\begin{aligned}
\mathcal{L}_{1} & =C_{a b c d} C^{a e f d} C_{e}{ }^{b c}{ }_{f}, \quad \mathcal{L}_{2}=C_{a b}{ }^{c d} C_{c d}{ }^{e f} C_{e f}{ }^{a b}, \\
\mathcal{L}_{3} & =C_{a b c d}\left(\delta_{e}^{a} \square-4 \mathcal{R}_{e}{ }^{a}+\frac{6}{5} \delta_{e}^{a} \mathcal{R}\right) C^{e b c d}
\end{aligned}
$$

where $C_{a b c d}$ is the Weyl tensor and $\mathcal{R}_{a b}$ is the Ricci tensor.

When superconformal field theories are under consideration, the type B anomalies should correspond to conformal supergravity invariants and generally the number of such invariants decreases with more supersymmetry. In six dimensions, superconformal algebras only exist for $\mathcal{N}=(n, 0)$ (or $\mathcal{N}=(0, n))$ [2], while the requirement that conformal supergravity does not contain higher spin fields limits $n<3$. Besides general interest in superconformal field theories, $\mathcal{N}=(2,0)$ models have been the focus of much interest due to their somewhat mysterious nature. Their existence was actually inferred by various arguments in string theory and they are believed to provide a description of the low-energy dynamics of multiple coincident M5-branes in M-theory.

In regards to the type B anomalies, there are two obvious questions. First, how many supersymmetric invariants are permitted and what linear combinations of (1.1) do they correspond to? Second, what are their fully supersymmetric forms when couplings to the rest of the Weyl multiplet of conformal supergravity are included? While very strong evidence exists for the purely gravitational form of these anomalies in the supersymmetric cases - namely the existence of two invariants in $(1,0)$ and only one for $(2,0)$, which we discuss below - very little was known about their supersymmetric completions. In principle the answer to both questions could be investigated via indirect means by e.g. computing the conformal anomaly of various $(1,0)$ or $(2,0)$ matter multiplets coupled to (super)gravity, as advocated in [3]. ${ }^{1}$ So far only the purely gravitational part of these computations have been performed in $6 \mathrm{D}$, see e.g. [6-8]. Alternatively, one could construct the full supersymmetric invariants directly.

Recently, the direct path was pursued in [9], where two $(1,0)$ Weyl invariants were built using superspace techniques, and a separate supercurrent analysis was given that established that there were at most two such invariants. One of them was observed to contain only $C^{3}$ terms in the particular combination

$$
-\frac{1}{8} \varepsilon^{a b c d e f} \varepsilon_{a^{\prime} b^{\prime} c^{\prime} d^{\prime} e^{\prime} f^{\prime}} C_{a b}{ }^{a^{\prime} b^{\prime}} C_{c d}{ }^{c^{\prime} d^{\prime}} C_{e f} e^{\prime f^{\prime}}=8 \mathcal{L}_{1}+4 \mathcal{L}_{2}
$$

while the other was observed to contain $\mathcal{L}_{3}$ at the quadratic order. As we will see, it actually contains additional cubic terms in the Weyl tensor in the particular combination

$$
4 \mathcal{L}_{1}-\mathcal{L}_{2}+\mathcal{L}_{3}
$$

We will refer to these two particular combinations as the $C^{3}$ and $C \square C$ invariants from now on.

\footnotetext{
${ }^{1}$ Such an approach was advocated for constructing the $4 \mathrm{D} \mathcal{N}=4$ conformal supergravity action [4] prior to its recent direct construction [5].
} 
The approach of [9] involved the direct construction of the two invariants from certain conformal primary superfields. These were composites built from the super-Weyl tensor and its derivatives within a novel superspace formulation of $6 \mathrm{D} \mathcal{N}=(1,0)$ conformal supergravity, called conformal superspace. Inspired by earlier formulations in three, four, and five dimensions [10-13], it is obtained by gauging the $6 \mathrm{D} \mathcal{N}=(1,0)$ superconformal algebra in superspace. As in those cases, the superspace torsion and curvature tensors turn out to be built solely in terms of the super-Weyl tensor, which simplifies computations significantly. ${ }^{2}$ Conformal superspace has proven useful in the context of general higherderivative supergravity actions, such as the $\mathcal{N}$-extended conformal supergravity actions in three dimensions for $3 \leq \mathcal{N} \leq 6$ [27] and a new set of curvature-squared invariants in $4 \mathrm{D} \mathcal{N}=2$ supergravity $[28,29]$, which arise from dimensional reduction of the $5 \mathrm{D}$ mixed gauge-gravity Chern-Simons term. A major advantage of conformal superspace is that at the component level, it recovers the Weyl multiplet and transformation rules of conformal supergravity as formulated within superconformal tensor calculus, first developed in six dimensions by Bergshoeff, Sezgin and Van Proeyen [30], building off earlier work in $4 \mathrm{D}[31,32]$ (see also the textbook [33]). The 6D superconformal tensor calculus has proven useful in the construction of the supersymmetric extension of a Riemann curvature squared term [34-36] and, more recently, in the complete off-shell action for minimal Poincaré supergravity [37]. Gauged minimal supergravity [38] has also been worked out by coupling the minimal Poincaré supergravity to an off-shell vector multiplet.

Our primary aims in this paper are to describe the connection between superspace and components for $6 \mathrm{D}(1,0)$ conformal superspace, and to convert the superspace invariants given in [9] to component form in the language of superconformal tensor calculus [30]. In particular, we will focus on the full set of (bosonic) terms that supersymmetrize (1.2) and (1.3). These are given respectively in (3.9) and (4.15). As another application, we present the component structure of an $F \square F$ invariant coupled to $(1,0)$ conformal supergravity with $F$ the field strength of a Yang-Mills multiplet. Its bosonic terms are given in (4.29) and coincide with the flat space construction of [39].

Although our main interest is in $(1,0)$ supersymmetry, it turns out one can deduce a great deal about the $(2,0)$ invariant once the structure of the $(1,0)$ invariants is known. Already quite a lot of evidence (see e.g. $[6,8,40]$ ) suggests that there should only be one type B anomaly with $(2,0)$ supersymmetry. Its purely gravitational part is

$$
4 \mathcal{L}_{1}+\mathcal{L}_{2}+\frac{1}{3} \mathcal{L}_{3}
$$

and should be extendable to some $(2,0)$ conformal supergravity invariant containing additional terms involving other fields of the Weyl multiplet. To our knowledge, no analysis of the off-shell supersymmetric extension of this term has been performed. Another goal of this paper is to make a major step towards solving this problem. By analyzing the structure of the two $(1,0)$ conformal supergravity invariants, we will show that only a certain combination can be lifted to $(2,0)$ conformal supergravity; this combination has (1.4) as

\footnotetext{
${ }^{2}$ In conventional superspace approaches, see e.g. [14-26], the structure group contains only the Lorentz and $R$-symmetry groups, and additional torsion superfields appear.
} 
its purely gravitational part. We exploit the uplift to $(2,0)$ to give for the first time a large part of the bosonic sector of the off-shell $(2,0)$ conformal supergravity invariant.

This paper is organized as follows. In section 2 we show how to recover the Weyl multiplet from conformal superspace and derive the associated supersymmetry transformations. In section 3 we describe the component structure of the $C^{3}$ invariant, explicitly giving its bosonic sector. In section 4 we consider the $C \square C$ and $F \square F$ invariants. We devote section 5 to a discussion of the off-shell $\mathcal{N}=(2,0)$ conformal supergravity invariant. Finally, we discuss our results and present some concluding remarks in section 6 .

We have also included a few technical appendices. In appendix A we provide the salient details of conformal superspace and describe how the covariant derivative algebra is deformed under a certain redefinition of the vector covariant derivative, which we will use in the main body of the paper. Appendix B provides a brief prescription for how to relate our notation and conventions to those already appearing in the literature. Along with the arXiv submission, we have included a separate supplementary file containing the building blocks (including all fermionic terms) for the $(1,0)$ invariants constructed in sections 3 and 4 .

\section{The $6 \mathrm{D} \mathcal{N}=(1,0)$ Weyl multiplet from superspace}

In this section, we will show how to reduce the superspace formulation of conformal supergravity in [9] to components. Let us first elaborate on the component structure of the Weyl multiplet of $6 \mathrm{D} \mathcal{N}=(1,0)$ conformal supergravity, first developed in [30]. Within the superconformal tensor calculus framework [30], one gauges the superconformal algebra in spacetime. Associated respectively with local translations, $Q$-supersymmetry, $\mathrm{SU}(2) R$ symmetry, and dilatations are the vielbein $e_{m}{ }^{a}$, the gravitino $\psi_{m_{i}}^{\alpha}$, the $\mathrm{SU}(2)$ gauge field $\mathcal{V}_{m}{ }^{i j}$, and a dilatation gauge field $b_{m}$. The remaining gauge symmetries are associated with composite connections: these are the spin connection $\omega_{m}{ }^{c d}$, the $S$-supersymmetry connection $\phi_{m} \underset{\alpha}{i}$, and the special conformal connection $\mathfrak{f}_{m a}$. To ensure that the last three connections are composite, one imposes conventional constraints (which are in general not unique) on the vielbein curvature $R(P)_{m n}{ }^{a}$, the gravitino curvature $R(Q)_{m n_{i}}^{\alpha}$, and the conformal Lorentz curvature $R(M)_{m n}{ }^{a b}$. However, the independent one-forms cannot furnish an off-shell representation of a conformal supersymmetry algebra as the bosonic and fermionic degrees of freedom do not match. An off-shell representation is achieved by introducing three covariant fields: a real anti-self-dual tensor $T_{a b c}^{-}$, a chiral fermion $\chi^{\alpha i}$, and a real scalar field $D$ which deform the supersymmetry algebra, the curvatures and the constraints imposed on the curvatures in a consistent way [30]. This procedure can be considered as a bottom-up approach where step-by-step one builds up a consistent off-shell multiplet for conformal supergravity.

In conformal superspace the superconformal algebra is manifestly gauged off-shell from the very beginning. Rather than construct a multiplet of gauge fields and covariant matter fields which must possess the same supersymmetry algebra (usually with modifications due to the curvature tensors and covariant fields), one must completely determine the supersymmetry algebra directly by solving superspace Bianchi identities. Typically these identities are solved by a single superfield, which encodes all component curvature tensors 
along with the covariant fields necessary for off-shell closure. Once the solution is found, the component fields and their supersymmetry transformations can be obtained directly by projecting to spacetime; the resulting component structure turns out to match that constructed in components directly. This method therefore can be viewed as a top-down approach.

\subsection{Component fields and curvatures from superspace}

We begin by identifying the various component fields of the $6 \mathrm{D} \mathcal{N}=(1,0)$ Weyl multiplet [30] within the geometry of conformal superspace [9]. For the one-forms, this identification is particularly easy as each component one-form is in direct correspondence with some superspace one-form and these can be connected in a straightforward way.

Let us start with the vielbein $\left(e_{m}{ }^{a}\right)$ and gravitino $\left(\psi_{m_{i}}^{\alpha}\right)$. These appear as the $\theta=0$ projections of the coefficients of $\mathrm{d} x^{m}$ in the supervielbein $E^{A}=\left(E^{a}, E_{i}^{\alpha}\right)=\mathrm{d} z^{M} E_{M}{ }^{A},{ }^{3}$

$$
e_{m}{ }^{a}(x):=E_{m}{ }^{a}(z)\left|, \quad \psi_{m_{i}}^{\alpha}(x):=2 E_{m_{i}}^{\alpha}(z)\right|,
$$

where a single vertical line next to a superfield denotes setting $\theta=0$. This operation can be written in a coordinate-independent way using the so-called double-bar projection [41, 42]

$$
e^{a}=\mathrm{d} x^{m} e_{m}^{a}=E^{a}\left\|, \quad \psi_{i}^{\alpha}=\mathrm{d} x^{m} \psi_{m_{i}}^{\alpha}=2 E_{i}^{\alpha}\right\|,
$$

where the double bar denotes setting $\theta=\mathrm{d} \theta=0 .{ }^{4}$ In like fashion, the remaining fundamental and composite one-forms correspond to double-bar projections of superspace one-forms,

$$
\mathcal{V}^{k l}:=\Phi^{k l}\|, \quad b:=B\|, \quad \omega^{c d}:=\Omega^{c d}\left\|, \quad \phi_{\gamma}^{k}:=2 \mathfrak{F}_{\gamma}^{k}\right\|, \quad \mathfrak{f}_{c}:=\mathfrak{F}_{c} \| .
$$

The covariant matter fields are contained within the super-Weyl tensor $W_{a b c}$ and its independent descendants. We define the three covariant component fields as ${ }^{5}$

$$
\begin{aligned}
T_{a b c}^{-} & :=-2 W_{a b c} \mid, \\
\chi^{\alpha i} & :=\frac{15}{2} X^{\alpha i}\left|=-\frac{3 \mathrm{i}}{4} \nabla_{\beta}^{i} W^{\alpha \beta}\right|, \\
D & :=\frac{15}{2} Y\left|=-\frac{3 \mathrm{i}}{16} \nabla_{\alpha}^{k} \nabla_{\beta k} W^{\alpha \beta}\right| .
\end{aligned}
$$

There are three additional independent descendant fields: the dimension- $3 / 2$ fermionic field $\mathcal{X}_{\alpha}^{i \beta \gamma}:=X_{\alpha}^{i \beta \gamma} \mid$, and the dimension-2 bosonic fields $\mathcal{Y}_{\alpha}{ }^{\beta k l}:=Y_{\alpha}{ }^{\beta k l} \mid$ and $\mathcal{Y}_{\alpha \beta}{ }^{\gamma \delta}:=Y_{\alpha \beta}{ }^{\gamma \delta} \mid{ }^{6}$ These will turn out to be composite and expressible directly in terms of the component

\footnotetext{
${ }^{3}$ Recall that $z^{M}=\left(x^{m}, \theta_{\imath}^{\mu}\right)$ are coordinates for a local parametrization of $6 \mathrm{D} \mathcal{N}=(1,0)$ conformal superspace, see [9] and appendix A.

${ }^{4}$ In more mathematical language, the double-bar projection is the pullback of the inclusion map embedding spacetime into superspace.

${ }^{5}$ We have chosen the coefficients such that $T_{a b c}^{-}, \chi^{\alpha i}$ and $D$ exactly correspond to the covariant matter fields of the Weyl multiplet introduced in [30]. We always denote the anti-self-dual covariant field as $T_{a b c}^{-}$ to avoid confusion with the superspace torsion tensor $T_{a b}{ }^{c}$.

${ }^{6}$ The descendant fields of $W_{a b c}$ are defined in appendix A.
} 
curvatures. The differential constraints on the superfield $W_{a b c}$ forbid any independent component fields at higher dimension [9].

It should be mentioned that one can impose a Wess-Zumino gauge to fix the $\theta$ expansions of the superspace gauge one-forms, so that they are completely determined by the above component fields. This ensures that the entire content of the superspace geometry is encoded in the independent physical fields. In practice, using the above definitions eliminates the need to do this explicitly.

In conformal superspace the covariant exterior derivative is defined as

$$
\nabla=E^{A} \nabla_{A}=\mathrm{d}-\frac{1}{2} \Omega^{c d} M_{c d}-B \mathbb{D}-\Phi^{k l} J_{k l}-\mathfrak{F}_{\alpha}^{i} S_{i}^{\alpha}-\mathfrak{F}_{a} K^{a},
$$

with $\nabla_{A}$ the covariant derivatives. By taking the double bar projection of $\nabla$, we can define the component $\nabla_{a}$ to coincide with the projection of the superspace derivative $\nabla_{a} \mid$,

$$
e_{m}{ }^{a} \nabla_{a}=\partial_{m}-\frac{1}{2} \psi_{m i}{ }^{\alpha} \nabla_{\alpha}^{i} \mid-\frac{1}{2} \omega_{m}{ }^{c d} M_{c d}-b_{m} \mathbb{D}-\mathcal{V}_{m}{ }^{k l} J_{k l}-\frac{1}{2} \phi_{m \alpha}{ }^{i} S_{i}^{\alpha}-\mathfrak{f}_{m a} K^{a} .
$$

The projected spinor covariant derivative $\nabla_{\alpha}^{i} \mid$ corresponds to the generator of $Q$ supersymmetry, and is defined so that if $\mathcal{U}=U \mid$, then $\nabla_{\alpha}^{i}\left|\mathcal{U}:=\left(\nabla_{\alpha}^{i} U\right)\right|$. Note that there is no ambiguity for the other generators as e.g. $M_{c d} \mathcal{U}=\left(M_{c d} U\right) \mid$, and so local diffeomorphisms, $Q$-supersymmetry transformations, and so forth descend naturally from their corresponding rule in superspace (A.11), which can be written

$$
\delta \mathcal{U}=\xi^{a} \nabla_{a} \mathcal{U}+\xi_{i}^{\alpha} \nabla_{\alpha}^{i} \mathcal{U}+\frac{1}{2} \lambda^{c d} M_{c d} \mathcal{U}+\lambda^{k l} J_{k l} \mathcal{U}+\sigma \mathbb{D} \mathcal{U}+\eta_{\gamma}^{k} S_{k}^{\gamma} \mathcal{U}+\lambda_{c} K^{c} \mathcal{U}
$$

Note that in spacetime we can choose to parametrize local superconformal transformations either with a covariant diffeomorphism, generated by $\xi^{a} \nabla_{a}$, or as a normal diffeomorphism, generated by $\xi^{m} \partial_{m}$. For $Q$-supersymmetry on the other hand, the natural choice in spacetime involves the covariant spinorial derivative $\nabla_{\alpha}^{i} \mid$ rather than the $\theta$-derivative.

The algebraic structure of these operators descends straightforwardly from superspace. For example, the component supercovariant curvature tensors arise by projecting (A.21c),

$$
\begin{aligned}
{\left[\nabla_{a}, \nabla_{b}\right]=} & -R(P)_{a b}{ }^{c} \nabla_{c}-R(Q)_{a b}^{\gamma}{ }_{k} \nabla_{\gamma}^{k} \mid-\frac{1}{2} R(M)_{a b}{ }^{c d} M_{c d}-R(J)_{a b}{ }^{k l} J_{k l} \\
& -R(\mathbb{D})_{a b} \mathbb{D}-R(S)_{a b \gamma}^{k} S_{k}^{\gamma}-R(K)_{a b c} K^{c},
\end{aligned}
$$

where we have introduced the expressions $R(P)_{a b}{ }^{c}=T_{a b}{ }^{c} \mid$ and $R(Q)_{a b k}^{\gamma}=T_{a b}{ }_{k}^{\gamma} \mid$ for the lowest components of the superspace torsion tensors to match the usual component nomenclature, while $R(M)_{a b}{ }^{c d}, R(J)_{a b}{ }^{k l}, R(\mathbb{D})_{a b}, R(S)_{a b \gamma}^{k}$ and $R(K)_{a b c}$ are the lowest components of the corresponding superspace curvatures.

The constraints on the superspace curvatures determine how the covariant fields of the Weyl multiplet should appear within these curvatures; in other words, the superspace geometry dictates how to supercovariantize a given component curvature. Let us illustrate this by deriving the explicit form of $R(P)_{a b}{ }^{c}:=T_{a b}{ }^{c}$. Consider the double bar projection of the torsion two-form $T^{c}$, eq. (A.7a). This can be evaluated either in terms of its explicit definition,

$$
T^{c}\left\|:=\mathcal{D} E^{c}\right\|=\mathcal{D} e^{c}=\mathrm{d} x^{n} \wedge \mathrm{d} x^{m} \mathcal{D}_{[m} e_{n]}^{c},
$$


where we have introduced the spin, dilatation, and $\mathrm{SU}(2)$ covariant derivative

$$
\mathcal{D}_{m}:=\partial_{m}-\frac{1}{2} \omega_{m}{ }^{c d} M_{c d}-b_{m} \mathbb{D}-\mathcal{V}_{m}{ }^{k l} J_{k l}, \quad \mathcal{D}_{a}:=e_{a}{ }^{m} \mathcal{D}_{m},
$$

or in terms of its tangent space decomposition

$$
\begin{aligned}
T^{c} \| & =\frac{1}{2} E^{A} \wedge E^{B} T_{B A^{c} \|} \\
& =\frac{1}{2} \mathrm{~d} x^{n} \wedge \mathrm{d} x^{m}\left(e_{m}{ }^{a} e_{n}{ }^{b} T_{a b}{ }^{c}\left|+e_{m}{ }^{a} \psi_{n_{j}}{ }^{\beta} T_{a_{\beta}}{ }^{c}{ }^{c}\right|-\frac{1}{4} \psi_{m_{i}}{ }^{\alpha} \psi_{n_{j}}{ }^{\beta} T_{\alpha \beta}^{i j}{ }^{i} \mid\right) .
\end{aligned}
$$

Equating (2.9) and (2.11) and solving for $R(P)_{a b}{ }^{c}$ leads to

$$
R(P)_{a b}{ }^{c}=2 e_{a}{ }^{m} e_{b}{ }^{n} \mathcal{D}_{[m} e_{n]}^{c}+\psi_{[a j}{ }^{\beta} T_{b]}{ }^{j}{ }^{c}\left|+\frac{1}{4} \psi_{[a i}{ }^{\alpha} \psi_{b] j}{ }^{\beta} T_{\alpha \beta}^{i j c}\right| .
$$

Proceeding in the same way for the other curvature two-forms gives

$$
\begin{aligned}
& R(Q)_{a b_{k}}^{\gamma}=\frac{1}{2} \Psi_{a b k}^{\gamma}+\mathrm{i}\left(\tilde{\gamma}_{[a}\right)^{\gamma \delta} \phi_{b] \delta k}+\psi_{\left[a_{j}\right.}^{\beta} T_{b]}^{j \gamma}{ }_{\beta k}^{j \gamma}\left|+\frac{1}{4} \psi_{a i}^{\alpha} \psi_{b_{j}}^{\beta} T_{\alpha \beta k}^{i j \gamma}\right|, \\
& R(\mathbb{D})_{a b}=2 e_{a}{ }^{m} e_{b}{ }^{n} \partial_{[m} b_{n]}+4 \mathfrak{f}_{[a b]}-\psi_{[a i}{ }^{\alpha} \phi_{b]}{ }_{\alpha}^{i}+\psi_{[a j}^{\beta} R(\mathbb{D})_{b]}{ }_{\beta}^{j} \mid \\
& +\frac{1}{4} \psi_{[a i}^{\alpha} \psi_{b]}^{\beta} R(\mathbb{D})_{\alpha \beta}^{i j} \mid \text {, } \\
& \left.R(M)_{a b}{ }^{c d}=\mathcal{R}_{a b}{ }^{c d}+8 \delta_{[a}^{[c} \mathfrak{f}_{b]}{ }^{d]}-\psi_{[a j}^{\alpha} \phi_{b]_{\beta}}^{j}\left(\gamma^{c d}\right)_{\alpha}{ }^{\beta}+\psi_{[a j}{ }^{\beta} R(M)_{b]}\right]_{\beta}^{j c d} \mid \\
& +\frac{1}{4} \psi_{a i}^{\alpha} \psi_{b_{j}}^{\beta} R(M)_{\alpha \beta}^{i j c d} \mid \\
& R(J)_{a b}{ }^{k l}=\mathcal{R}_{a b}{ }^{k l}+4 \psi_{[a}{ }^{\gamma(k} \phi_{b] \gamma}^{l)}+\psi_{[a j}^{\beta} R(J)_{b]_{\beta}}^{j k l}\left|+\frac{1}{4} \psi_{a_{i}}{ }^{\alpha} \psi_{b j}^{\beta} R(J)_{\alpha \beta}^{i j k l}\right|,
\end{aligned}
$$

where

$$
\begin{aligned}
\Psi_{a b k}^{\gamma} & :=2 e_{a}{ }^{m} e_{b}{ }^{n} \mathcal{D}_{[m} \psi_{n] k}^{\gamma}, \\
\mathcal{R}_{a b}{ }^{c d} & :=\mathcal{R}_{a b}{ }^{c d}(\omega)=e_{a}{ }^{m} e_{b}{ }^{n}\left(2 \partial_{[m} \omega_{n]}{ }^{c d}-2 \omega_{[m}{ }^{c e} \omega_{n] e^{d}}\right), \\
\mathcal{R}_{a b}{ }^{k l} & :=\mathcal{R}_{a b}{ }^{k l}(\mathcal{V})=e_{a}{ }^{m} e_{b}{ }^{n}\left(2 \partial_{[m} \mathcal{V}_{n]}{ }^{k l}+2 \mathcal{V}_{[m}{ }^{p(k} \mathcal{V}_{n] p}{ }^{l)}\right) .
\end{aligned}
$$

The last two terms in each of the curvatures (2.12) involve the covariant fields of the Weyl multiplet and from a component perspective are necessary for off-shell superconformal covariance. From a superspace perspective, their structure is instead dictated by the superspace geometry. Using the torsion and curvatures of [9], their explicit forms are

$$
\begin{aligned}
R(P)_{a b}{ }^{c} & =2 e_{a}{ }^{m} e_{b}{ }^{n} \mathcal{D}_{[m} e_{n]}^{c}+\frac{\mathrm{i}}{2} \psi_{[a i} \gamma^{c} \psi_{b]}{ }^{i} \\
R(Q)_{a b k} & =\frac{1}{2} \Psi_{a b k}+\mathrm{i} \tilde{\gamma}_{[a} \phi_{b] k}+\frac{1}{12} T_{c d e}^{-} \tilde{\gamma}^{c d e} \gamma_{[a} \psi_{b] k} \\
R(\mathbb{D})_{a b} & =2 e_{a}{ }^{m} e_{b}{ }^{n} \partial_{[m} b_{n]}+4 \mathfrak{f}_{[a b]}-\psi_{[a i} \phi_{b]}{ }^{i}-\frac{\mathrm{i}}{6} \psi_{[a j} \gamma_{b]} \chi^{j} \\
R(M)_{a b}{ }^{c d} & =\mathcal{R}_{a b}{ }^{c d}+8 \delta_{[a}^{[c} \mathfrak{f}_{b]}{ }^{d]}-\psi_{[a j} \gamma^{c d} \phi_{b]}{ }^{j}+2 \mathrm{i} \psi_{[a j} \gamma_{b]} R(Q)^{c d j}-\frac{\mathrm{i}}{6} \psi_{[a j} \gamma_{b]} \tilde{\gamma}^{c d} \chi^{j} \\
R(J)_{a b}{ }^{k l} & =\mathcal{R}_{a b}{ }^{k l}+4 \psi_{[a}{ }^{(k} \phi_{b]}{ }^{l)}+\frac{2 \mathrm{i}}{3} \psi_{[a}{ }^{(k} \gamma_{b]} \chi^{l)}
\end{aligned}
$$


Here we have suppressed spinor indices for legibility. Note that in $(2.14 \mathrm{~d})$ we have used the fact that the component field $\mathcal{X}_{\alpha}^{k \beta \gamma}$ turns out to be composite

$$
\left(\gamma_{a b}\right)_{\beta}{ }^{\alpha} \mathcal{X}_{\alpha}^{k \beta \gamma}=R(Q)_{a b}{ }^{\gamma k}-\frac{1}{10}\left(\tilde{\gamma}_{a b}\right)^{\gamma}{ }_{\beta} \chi^{\beta k}
$$

For the sake of brevity, we do not present here the expressions for $R(S)_{a b}{ }^{k}$ and $R(K)_{a b c}$.

\subsection{Analysis of the curvature constraints}

It has already been mentioned that the component spin, $S$-supersymmetry, and special conformal connections turn out to be composite. This property arises here just as in the purely component framework [30] because of constraints on some of the curvature tensors. In our framework, these constraints are already imposed at the superfield level and lead to

$$
\begin{aligned}
R(P)_{a b}^{c} & =2 T_{a b}^{-c}, \\
\gamma^{b} R(Q)_{a b k} & =\frac{1}{2} \gamma_{a} \chi_{k}, \\
R(M)_{a b}{ }^{c b} & =-\frac{1}{3} \delta_{a}^{c} D+\nabla^{b} T_{b a}^{-c},
\end{aligned}
$$

where

$$
\nabla_{d} T_{a b c}^{-}=\mathcal{D}_{d} T_{a b c}^{-}+\frac{\mathrm{i}}{15}\left(\gamma_{a b c}\right)_{\alpha \beta} \psi_{d k}^{\alpha} \chi^{\beta k}+\frac{\mathrm{i}}{2}\left(\gamma_{a b c}\right)_{\alpha \beta} \psi_{d k}^{\gamma} \mathcal{X}_{\gamma}^{k \alpha \beta}
$$

The first constraint (2.16a) determines the spin connection to be

$$
\omega_{a b c}=\omega(e)_{a b c}-2 \eta_{a[b} b_{c]}-\frac{\mathrm{i}}{4} \psi_{b}^{k} \gamma_{a} \psi_{c k}-\frac{\mathrm{i}}{2} \psi_{a}^{k} \gamma_{[b} \psi_{c] k}+T_{a b c}^{-},
$$

where $\omega(e)_{a b c}=-\frac{1}{2}\left(\mathcal{C}_{a b c}+\mathcal{C}_{c a b}-\mathcal{C}_{b c a}\right)$ is the usual torsion-free spin connection given in terms of the anholonomy coefficient $\mathcal{C}_{m n}{ }^{a}:=2 \partial_{[m} e_{n]}{ }^{a}$. It is important to note that the spin connection $\omega_{a b c}$ possesses not only the usual fermionic torsion, due to the gravitino terms, but also bosonic torsion from the covariant field $T_{a b c}^{-}$. In particular, this means that there is non-trivial dependence on $T_{a b c}^{-}$nested in every covariant derivative $\nabla_{a}$ and $\mathcal{D}_{a}$.

The second constraint $(2.16 \mathrm{~b})$ is solved by

$$
\phi_{m}{ }^{k}=\frac{\mathrm{i}}{16}\left(\gamma^{b c} \gamma_{m}-\frac{3}{5} \gamma_{m} \tilde{\gamma}^{b c}\right)\left(\Psi_{b c}^{k}+\frac{1}{6} T_{d e f}^{-} \tilde{\gamma}^{d e f} \gamma_{[b} \psi_{c]}^{k}\right)-\frac{\mathrm{i}}{10} \gamma_{m} \chi^{k}
$$

Reinserting this into the original expression for $R(Q)$ gives

$$
R(Q)_{a b k}=\frac{1}{2} \Pi_{a b} c d\left(\Psi_{c d k}+\frac{1}{6} T_{e f g}^{-} \tilde{\gamma}^{e f g} \gamma_{[c} \psi_{d] k}\right)+\frac{1}{10} \tilde{\gamma}_{a b} \chi_{k}
$$

where $\Pi_{a b} c d$ is the projection operator onto gamma-traceless spinor-valued two-forms, ${ }^{7}$

$$
\begin{aligned}
\Pi_{a b}{ }^{c d} & :=\frac{3}{5} \delta_{a}^{[c} \delta_{b}^{d]}+\frac{3}{10} \delta_{[a}^{[c} \tilde{\gamma}_{b]}^{d]}+\frac{1}{40} \varepsilon_{a b}{ }^{c d e f} \tilde{\gamma}_{e f}, \\
\gamma^{a} \Pi_{a b}{ }^{c d} & =\Pi_{a b}{ }^{c d} \tilde{\gamma}_{c}=0, \quad \Pi_{a b}{ }^{e f} \Pi_{e f}{ }^{c d}=\Pi_{a b}{ }^{c d} .
\end{aligned}
$$

\footnotetext{
${ }^{7}$ For the projection operator $\Pi_{a b}{ }^{c d}$ in other dimensions, see also e.g. [13, 43].
} 
Note that eq. (2.15) can then be expressed as

$$
\mathcal{X}_{a b}{ }^{\gamma k}:=\frac{1}{2}\left(\gamma_{a b}\right)_{\beta}{ }^{\alpha} \mathcal{X}_{\alpha}^{k \beta \gamma}=\frac{1}{2}\left(\Pi_{a b}{ }^{c d}\right)^{\gamma}{ }_{\delta} R(Q)_{c d}{ }^{\delta k} .
$$

This relates the field $\mathcal{X}_{\alpha}^{k \beta \gamma}$ to the $\gamma$-traceless part of the gravitino field strength.

The third constraint (2.16c) is solved by

$$
\begin{aligned}
\mathfrak{f}_{a}{ }^{b}= & -\frac{1}{8} \mathcal{R}_{a}{ }^{b}(\omega)+\frac{1}{80} \delta_{a}^{b} \mathcal{R}(\omega)-\frac{1}{60} \delta_{a}^{b} D+\frac{1}{8} \nabla^{c} T_{c a}^{-b} \\
& +\frac{\mathrm{i}}{8} \psi_{c j} \gamma_{a} R(Q)^{b c j}+\frac{1}{8} \psi_{[a j} \gamma^{b c} \phi_{c]}^{j}-\frac{1}{80} \delta_{a}^{b} \psi_{c j} \gamma^{c d} \phi_{d}{ }^{j} \\
& -\frac{\mathrm{i}}{96} \psi_{c j} \gamma_{a}^{b c} \chi^{j}-\frac{\mathrm{i}}{48} \psi_{a j} \gamma^{b} \chi^{j}+\frac{\mathrm{i}}{80} \delta_{a}^{b} \psi_{c j} \gamma^{c} \chi^{j},
\end{aligned}
$$

where we have defined $\mathcal{R}_{a}{ }^{b}(\omega):=\mathcal{R}_{a c}{ }^{b c}(\omega)$ and $\mathcal{R}(\omega):=\mathcal{R}_{a}{ }^{a}(\omega)$. Inserting this back into $R(M)_{a b}^{c d}$ leads to a quite involved expression; its bosonic part is

$$
R(M)_{a b}{ }^{c d}=C(\omega)_{a b}{ }^{c d}-\frac{2}{15} \delta_{a}^{[c} \delta_{b}^{d]} D+\mathcal{D}^{e} T_{e[a}^{-[c} \delta_{b]}^{d]}+(\text { explicit gravitino terms }) .
$$

Here $C(\omega)_{a b}{ }^{c d}:=\mathcal{R}(\omega)_{a b}{ }^{c d}-\delta_{[a}^{[c} \mathcal{R}(\omega)_{b]}{ }^{d]}+\frac{1}{10} \delta_{[a}^{[c} \delta_{b]}^{d]} \mathcal{R}(\omega)$ is the traceless part of the tensor $\mathcal{R}(\omega)_{a b}{ }^{c d}$. It is important to observe that $C(\omega)_{a b}{ }^{c d}$ is not quite the usual component Weyl tensor due to the presence of the bosonic torsion in the spin connection.

We have already mentioned that the super-Weyl tensor superfield includes, besides the covariant matter fields, three other independent descendants, which turn out to be composite. The dimension-3/2 fermionic field $\mathcal{X}_{\alpha}^{i \beta \gamma}$ was already analyzed, see eq. (2.22), and is related to the gamma-traceless part of $R(Q)_{a b}{ }^{\alpha i}$. The dimension-2 bosonic fields $\mathcal{Y}_{a b}{ }^{c d}:=\frac{1}{4}\left(\gamma_{a b}\right)_{\gamma}{ }^{\alpha}\left(\gamma^{c d}\right)_{\delta}{ }^{\beta} \mathcal{Y}_{\alpha \beta}{ }^{\gamma \delta}$ and $\mathcal{Y}_{a b}{ }^{k l}:=\frac{1}{2}\left(\gamma_{a b}\right)_{\beta}{ }^{\alpha} \mathcal{Y}_{\alpha}{ }^{\beta k l}$ are given respectively by the traceless part of $R(M)_{a b} c d$ and the $\mathrm{SU}(2)$ curvature $R(J)_{a b}{ }^{i j}$,

$$
\begin{aligned}
& \mathcal{Y}_{a b}{ }^{c d}=R(M)_{a b}{ }^{c d}+\frac{2}{15} \delta_{a}^{[c} \delta_{b}^{d]} D-2 \nabla_{[a} T_{b]}^{-c d}-2 \nabla^{e} T_{e[a}^{-[c} \delta_{b]}^{d]}, \\
& \mathcal{Y}_{a b}{ }^{k l}=R(J)_{a b}{ }^{k l} .
\end{aligned}
$$

It should be mentioned that the constraint $R(\mathbb{D})_{a b}=\nabla^{c} T_{a b c}^{-}$, derived from superspace, actually holds identically after substituting the expression for $\mathfrak{f}_{m c}$ into $R(\mathbb{D})$. Although we do not provide the analysis here, the same is true for the $R(S)$ and $R(K)$ curvatures, which are determined in terms of the other curvatures due to Bianchi identities. We will return to this point at the end of the next subsection.

Now let us note an interesting feature of the expressions (2.16). In contrast to the constraints employed in [30], these are $S$-invariant. The reason is that the superspace constraints of [9] were chosen so that the superspace derivatives $\nabla_{\alpha}^{i}$ and $\nabla_{a}$ have the same algebra with $S_{i}^{\alpha}$ and $K^{a}$ as in the $6 \mathrm{D} \mathcal{N}=(1,0)$ superconformal algebra. This simplicity comes with the price that the composite connections have nontrivial dependence on the fields $T_{a b c}^{-}, \chi^{i}$ and $D$. This renders component expressions more involved than one might desire. Therefore, instead of completing the analysis here and deriving the supersymmetry transformations etc., we will make a different choice of conventional constraints to remove the dependence on the covariant fields from the connections. 


\subsection{Different choices of conventional constraints}

Let us consider the following redefinitions of the composite connections,

$$
\begin{aligned}
\hat{\omega}_{m}^{b c} & =\omega_{m}^{b c}-\frac{1}{2} \lambda_{1} e_{m}{ }^{a} T_{a}^{-b c}, \\
\hat{\phi}_{m}^{j} & =\phi_{m}^{j}-\frac{4 \mathrm{i}}{15} \lambda_{2}\left(\gamma_{m}\right)_{\beta \gamma} \chi^{\gamma j}, \\
\hat{\mathfrak{f}}_{m b} & =\mathfrak{f}_{m b}+e_{m}{ }^{a}\left(\frac{2}{15} \lambda_{3} \eta_{a b} D-\frac{1}{2} \lambda_{4} \nabla^{c} T_{a c b}^{-}+\frac{1}{4} \lambda_{5} T_{a}^{-c d} T_{c d b}^{-}\right),
\end{aligned}
$$

where $\lambda_{1}, \lambda_{2}, \lambda_{3}, \lambda_{4}$ and $\lambda_{5}$ are real constant parameters. A specific choice can eliminate the covariant matter fields from the connections, but let us remain more general for the moment. These redefinitions can be interpreted as a change of frame in superspace by redefining the vector covariant derivative

$$
\begin{aligned}
\hat{\nabla}_{a}= & \nabla_{a}-\frac{1}{2} \lambda_{1} W_{a}{ }^{b c} M_{b c}-\mathrm{i} \lambda_{2}\left(\gamma_{a}\right)_{\alpha \beta} X^{\alpha j} S_{j}^{\beta}-\lambda_{3} Y K_{a} \\
& -\lambda_{4} \nabla^{b} W_{a b c} K^{c}-\lambda_{5} W_{a}{ }^{e f} W_{e f c} K^{c}
\end{aligned}
$$

while keeping the spinor covariant derivative the same $\hat{\nabla}_{\alpha}^{i}=\nabla_{\alpha}^{i}$. A detailed analysis of the modifications to the conformal superspace geometry associated with $\hat{\nabla}_{A}$ is relegated to appendix A. Here we focus on the implications for the component structures.

First of all, by comparing (2.18) and (2.26a), it is clear that the dependence on $T_{a b c}^{-}$ in the spin connection can be eliminated by choosing

$$
\lambda_{1}=2 \quad \Longrightarrow \quad \hat{R}(P)_{a b}{ }^{c}=0
$$

eliminating the bosonic torsion. In terms of this new spin connection,

$$
\mathfrak{f}_{a}{ }^{b}=-\frac{1}{8} \mathcal{R}_{a}{ }^{b}(\hat{\omega})+\frac{1}{80} \delta_{a}^{b} \mathcal{R}(\hat{\omega})+\frac{1}{4} \nabla^{c} T_{c a}^{-b}-\frac{1}{8} T_{a c d}^{-} T^{-c d b}-\frac{1}{60} \delta_{a}^{b} D+\cdots,
$$

where $\mathcal{R}_{a}{ }^{b}(\hat{\omega})$ and $\mathcal{R}(\hat{\omega})$ are defined in terms of the tensor $\mathcal{R}_{a b}{ }^{c d}(\hat{\omega})$ as in eq. (2.13b). Now shifting $\mathfrak{f}_{m c} \rightarrow \hat{\mathfrak{f}}_{m c}$ as in (2.26c) with $\lambda_{4}=-\frac{1}{2}$, we can get rid of the term $\nabla^{c} T_{c a}^{-b}$. In what follows, we will keep the choices $\lambda_{1}=2$ and $\lambda_{4}=-\frac{1}{2}$ fixed.

It is now straightforward to reapply the same component reduction procedure of the previous subsection but in the "hat" frame. Using (2.12a)-(2.12e) together with (A.28)- 
(A.30) leads to ${ }^{8}$

$$
\begin{aligned}
\hat{R}(P)_{a b}{ }^{c}= & 0 \\
\hat{R}(Q)_{a b k}= & \frac{1}{2} \hat{\Psi}_{a b k}+\mathrm{i} \tilde{\gamma}_{[a} \hat{\phi}_{b] k}+\frac{1}{24} T_{c d e}^{-} \tilde{\gamma}^{c d e} \gamma_{[a} \psi_{b] k} \\
\hat{R}(\mathbb{D})_{a b}= & 2 e_{a}{ }^{m} e_{b}{ }^{n} \partial_{[m} b_{n]}+4 \hat{\mathfrak{f}}_{[a b]}+\psi_{[a}{ }^{i} \hat{\phi}_{b] i}+\frac{4 \mathrm{i}}{15}\left(\frac{5}{8}+\lambda_{2}\right) \psi_{[a}^{j} \gamma_{b]} \chi_{j}, \\
\hat{R}(M)_{a b}{ }^{c d}= & \left.\mathcal{R}_{a b}{ }^{c d}(\hat{\omega})+8 \delta_{[a}^{\left[c \hat{\mathfrak{f}}_{b]} d\right]}+\mathrm{i} \psi_{[a j} \gamma_{b]} \hat{R}(Q)^{c d j}+2 \mathrm{i} \psi_{[a j} \gamma^{[c} \hat{R}(Q)_{b]} d\right] j \\
& -\psi_{[a j} \gamma^{c d} \hat{\phi}_{b]}^{j}-\frac{8 \mathrm{i}}{15}\left(\frac{5}{8}+\lambda_{2}\right) \delta_{[a}^{[c} \psi_{b] j} \gamma^{d]} \chi^{j}+\frac{\mathrm{i}}{2} \psi_{[a}^{j} \gamma^{e} \psi_{b] j} T_{e}^{-c d}, \\
\hat{R}(J)_{a b}{ }^{k l}= & \mathcal{R}_{a b}{ }^{k l}(\mathcal{V})+4 \psi_{[a}{ }^{(k} \hat{\phi}_{b]}^{l)}+\frac{16 \mathrm{i}}{15}\left(\frac{5}{8}+\lambda_{2}\right) \psi_{[a}{ }^{(k} \gamma_{b]} \chi^{l)},
\end{aligned}
$$

where we have introduced the derivatives

$$
\hat{\mathcal{D}}_{m}=\partial_{m}-\frac{1}{2} \hat{\omega}_{m}{ }^{b c} M_{b c}-b_{m} \mathbb{D}-\mathcal{V}_{m}{ }^{i j} J_{i j}, \quad \hat{\mathcal{D}}_{a}=e_{a}{ }^{m} \hat{\mathcal{D}}_{m},
$$

together with the gravitini field strength, $\hat{\Psi}_{a b}^{\gamma}=e_{a}{ }^{m} e_{b}{ }^{n} \hat{\mathcal{D}}_{[m} \psi_{n] k}^{\gamma}$ as in (2.13a). The new superspace curvature constraints now lead to

$$
\begin{aligned}
\hat{R}(P)_{a b}{ }^{c} & =0 \\
\gamma^{b} \hat{R}(Q)_{a b k} & =\frac{4}{3}\left(\lambda_{2}+\frac{3}{8}\right) \gamma_{a} \chi_{k}, \\
\hat{R}(M)_{a c}^{b c} & =\frac{8}{3}\left(\lambda_{3}-\frac{1}{8}\right) \delta_{a}^{b} D+2\left(\lambda_{5}-\frac{1}{2}\right) T_{a c d}^{-} T^{-b c d} .
\end{aligned}
$$

These constraints are no longer $S$-invariant. This is a consequence of the redefinition of the composite connections, which deforms their $S$-supersymmetry transformations. It is interesting to observe that for all values of $\lambda_{2}, \lambda_{3}$ and $\lambda_{5}$, the new dilatation curvature is zero while the $\mathrm{SU}(2)$ curvature is unchanged,

$$
\hat{R}(\mathbb{D})_{a b}=0, \quad \hat{R}(J)_{a b}^{k l}=R(J)_{a b}^{k l}=\frac{1}{2}\left(\gamma_{a b}\right)_{\beta}{ }^{\alpha} \mathcal{Y}_{\alpha}{ }^{\beta k l} .
$$

The constraints (2.32) are solved by

$$
\begin{aligned}
\hat{\omega}_{a b c}= & \omega(e)_{a b c}-2 \eta_{a[b} b_{c]}-\frac{\mathrm{i}}{4} \psi_{b}{ }^{k} \gamma_{a} \psi_{c k}-\frac{\mathrm{i}}{2} \psi_{a}{ }^{k} \gamma_{[b} \psi_{c] k}, \\
\hat{\phi}_{m}{ }^{k}= & \frac{\mathrm{i}}{16}\left(\gamma^{b c} \gamma_{m}-\frac{3}{5} \gamma_{m} \tilde{\gamma}^{b c}\right)\left(\hat{\Psi}_{b c}{ }^{k}+\frac{1}{12} T_{d e f}^{-} \tilde{\gamma}^{d e f} \gamma_{[b} \psi_{c]}^{k}\right)-\frac{4 \mathrm{i}}{15}\left(\frac{3}{8}+\lambda_{2}\right) \gamma_{m} \chi^{k}, \quad \text { (2.34b) } \\
\hat{\mathfrak{f}}_{a}{ }^{b}= & -\frac{1}{8} \mathcal{R}_{a}{ }^{b}(\hat{\omega})+\frac{1}{80} \delta_{a}^{b} \mathcal{R}(\hat{\omega})+\frac{2}{15}\left(\lambda_{3}-\frac{1}{8}\right) \delta_{a}^{b} D+\frac{1}{4}\left(\lambda_{5}-\frac{1}{2}\right) T_{a e f}^{-} T^{-b e f}+\frac{1}{8} \psi_{[a j} \gamma^{b c} \hat{\phi}_{c]}{ }^{j} \\
& -\frac{1}{80} \delta_{a}^{b} \psi_{c j} \gamma^{c d} \hat{\phi}_{d}{ }^{j}+\frac{\mathrm{i}}{16} \psi_{c j} \gamma_{a} \hat{R}(Q)^{b c j}+\frac{\mathrm{i}}{8} \psi_{c j} \gamma^{[b} \hat{R}(Q)_{a}{ }^{c] j}-\frac{\mathrm{i}}{10}\left(\frac{5}{24}+\lambda_{2}\right) \psi_{a j} \gamma^{b} \chi^{j} \\
& +\frac{\mathrm{i}}{30}\left(\frac{3}{8}+\lambda_{2}\right) \delta_{a}^{b} \psi_{c j} \gamma^{c} \chi^{j}+\frac{\mathrm{i}}{16} \psi_{a}^{j} \gamma_{c} \psi_{d j} T^{-b c d}-\frac{\mathrm{i}}{160} \delta_{a}^{b} \psi_{c}^{j} \gamma_{d} \psi_{e j} T^{-c d e} .
\end{aligned}
$$

One may confirm that these are equivalent to (2.26) with $\lambda_{1}=2$ and $\lambda_{4}=-\frac{1}{2}$.

\footnotetext{
${ }^{8}$ As discussed in appendix A, the structure functions $\hat{f}_{\underline{a} b} \underline{c}$ may induce nontrivial corrections to the new curvatures. In our case, the $\left[S_{i}^{\alpha}, \hat{\nabla}_{a}\right]$ commutator is deformed, but induces modifications only in the expressions for the $\hat{R}(S)$ and $\hat{R}(K)$ curvatures. Up to hats, eqs. (2.12a)-(2.12e) are formally unchanged and apply also to the general frame.
} 
Reinserting the composite $S$-supersymmetry connection (2.34b) into eq. (2.30b) gives

$$
\hat{R}(Q)_{a b k}=\frac{1}{2} \Pi_{a b}{ }^{c d}\left(\hat{\Psi}_{c d k}+\frac{1}{12} T_{e f g}^{-} \tilde{\gamma}^{e f g} \gamma_{[c} \psi_{d] k}\right)+\frac{4}{15}\left(\frac{3}{8}+\lambda_{2}\right) \tilde{\gamma}_{a b} \chi_{k} .
$$

In the new frame the component field $\mathcal{X}_{a b}{ }^{\gamma k}:=\frac{1}{2}\left(\gamma_{a b}\right)_{\beta}{ }^{\alpha} \mathcal{X}_{\alpha}^{k \beta \gamma}$ becomes

$$
\begin{aligned}
\mathcal{X}_{a b}{ }^{k} & =\frac{1}{2} \hat{R}(Q)_{a b}^{k}-\frac{2}{15}\left(\frac{3}{8}+\lambda_{2}\right) \tilde{\gamma}_{a b} \chi^{k} \\
& =\frac{1}{2} \Pi_{a b} c d \hat{R}(Q)_{c d}{ }^{k}=\frac{1}{4} \Pi_{a b} c d\left(\hat{\Psi}_{c d}^{k}+\frac{1}{12} T_{e f g}^{-} \tilde{\gamma}^{e f g} \gamma_{[c} \psi_{d]}^{k}\right) .
\end{aligned}
$$

It is important to note that the component field $\mathcal{X}_{\alpha}^{k \beta \gamma}$ is unchanged in going to the new frame; that is, the equations (2.22) and (2.36) are completely equivalent - only the definition of $\hat{R}(Q)$ has changed. Other useful relations, which follow from the constraint (2.32b), are

$$
\begin{aligned}
\tilde{\gamma}^{a b} \hat{R}(Q)_{a b k} & =-8\left(\lambda_{2}+\frac{3}{8}\right) \chi_{k}, \\
\gamma^{c d e} \hat{R}(Q)_{b e}{ }^{k} & =2 \gamma^{[c} \hat{R}(Q)_{b}{ }^{d] k}+\frac{4}{3}\left(\lambda_{2}+\frac{3}{8}\right) \gamma^{c d} \gamma_{b} \chi^{k}, \\
\gamma^{e f} \gamma_{a b c} \hat{R}(Q)_{e f}{ }^{k} & =-24 \gamma_{[a} \hat{R}(Q)_{b c]}^{k}+8\left(\lambda_{2}+\frac{3}{8}\right) \gamma_{a b c} \chi^{k}, \\
\gamma_{[a} \hat{R}(Q)_{b c]}^{k} & =-\frac{1}{6} \varepsilon_{a b c d e f} \gamma^{[d} \hat{R}(Q)^{e f] k}
\end{aligned}
$$

It is clear that a particularly simple choice of frame is

$$
\lambda_{2}=-\frac{3}{8} \quad \Longrightarrow \quad \hat{R}(Q)_{a b k}=\frac{1}{2} \Pi_{a b} c d\left(\hat{\Psi}_{c d k}+\frac{1}{12} T_{e f g}^{-} \tilde{\gamma}^{e f g} \gamma_{[c} \psi_{d] k}\right)
$$

In this case the $\hat{R}(Q)_{a b k}$ curvature is $\gamma$-traceless. On the other hand, it can be proven that the choice $\lambda_{2}=-\frac{5}{16}$ corresponds to the conventional constraint for $\hat{R}(Q)$ that was employed in [30]; we refer the reader to appendix B for more details.

In principle, one can also reinsert the expression (2.34c) for $\hat{\mathfrak{f}}_{m c}$ into $\hat{R}(M)_{a b}{ }^{c d}$; in practice, we are mainly interested in the bosonic terms. These are

$$
\begin{aligned}
\hat{R}(M)_{a b}{ }^{c d}= & C(\hat{\omega})_{a b}{ }^{c d}+\frac{16}{15}\left(\lambda_{3}-\frac{1}{8}\right) \delta_{a}^{[c} \delta_{b}^{d]} D+2\left(\lambda_{5}-\frac{1}{2}\right) \delta_{[a}^{[c} T_{b] e f}^{-} T^{-d] e f} \\
& +(\text { explicit gravitino terms })
\end{aligned}
$$

where $C(\hat{\omega})_{a b}{ }^{c d}:=\mathcal{R}(\hat{\omega})_{a b}{ }^{c d}-\delta_{[a}^{[c} \mathcal{R}(\hat{\omega})_{b]}{ }^{d]}+\frac{1}{10} \delta_{[a}^{[c} \delta_{b]}^{d]} \mathcal{R}(\hat{\omega})$ coincides with the usual Weyl tensor when $\psi_{m}{ }^{i}$ and $b_{m}$ vanish. This implies that

$$
\begin{aligned}
\mathcal{Y}_{a b}{ }^{c d} & =\hat{R}(M)_{a b}{ }^{c d}-\frac{16}{15}\left(\lambda_{3}-\frac{1}{8}\right) \delta_{a}^{[c} \delta_{b}^{d]} D-2\left(\lambda_{5}-\frac{1}{2}\right) T_{a b e}^{-} T^{-e c d} \\
& =C(\hat{\omega})_{a b}{ }^{c d}+(\text { explicit gravitino terms }) .
\end{aligned}
$$




\begin{tabular}{|ccc|}
\hline & Traceless & Bergshoeff et al. \\
\hline$\lambda_{1}$ & 2 & 2 \\
$\lambda_{2}$ & $-\frac{3}{8}$ & $-\frac{5}{16}$ \\
$\lambda_{3}$ & $\frac{1}{8}$ & $\frac{5}{32}$ \\
$\lambda_{4}$ & $-\frac{1}{2}$ & $-\frac{1}{2}$ \\
$\lambda_{5}$ & $\frac{1}{2}$ & 1 \\
\hline
\end{tabular}

Table 1. Choice of parameters.

A special choice of $\lambda_{3}$ and $\lambda_{5}$ makes the Lorentz curvature traceless,

$$
\lambda_{3}=\frac{1}{8}, \quad \lambda_{5}=\frac{1}{2} \quad \Longrightarrow \quad \hat{R}(M)_{a b}{ }^{c d}=\mathcal{Y}_{a b}{ }^{c d}=C(\hat{\omega})_{a b}{ }^{c d}+\cdots .
$$

The particular choices of $\lambda_{2}, \lambda_{3}$ and $\lambda_{5}$ we have discussed maximally simplify the component curvatures and connections. We will refer to this choice as the "traceless" frame. It is associated with the following conventional constraints

$$
\hat{R}(P)_{a b}^{c}=0, \quad \gamma^{b} \hat{R}(Q)_{a b k}=0, \quad \hat{R}(M)_{a c}^{b c}=0 .
$$

Note that an alternative choice recovers the conventional constraints employed by Bergshoeff et al. [30], up to changes in notation described in appendix B. We summarize these two particular choices in table 1 .

So far we have not considered the curvatures $\hat{R}(S)$ and $\hat{R}(K)$ in detail. In principle, one could find explicit expressions for them in terms of $\hat{\phi}_{m_{\alpha}}^{i}$ and $\hat{\mathfrak{f}}_{m c}$. In practice, such expressions are not particularly useful since these connections and their curvatures are always composite quantities. Instead, it is more convenient to follow the component technique of analyzing the component Bianchi identities, which in our case is equivalent to performing the component projection of the corresponding superspace curvatures. Projecting (A.30d) and using (2.36) gives

$$
\begin{aligned}
\hat{R}(S)_{a b}{ }^{k}= & -\frac{\mathrm{i}}{2} \hat{\nabla} \hat{R}(Q)_{a b}{ }^{k}-\frac{\mathrm{i}}{3} \gamma_{[a} \hat{\nabla}^{c} \hat{R}(Q)_{b] c}{ }^{k}+\frac{\mathrm{i}}{3} T_{c d[a}^{-} \gamma^{c} \hat{R}(Q)_{b]} d k \\
& +\frac{2 \mathrm{i}}{9}\left(\lambda_{2}+\frac{3}{8}\right) \gamma_{c} \tilde{\gamma}_{a b} \hat{\nabla}^{c} \chi^{k}+\frac{2 \mathrm{i}}{9}\left(\lambda_{2}+\frac{3}{8}\right) T_{a b c}^{-} \gamma^{c} \chi^{k} .
\end{aligned}
$$

In a general frame, the special conformal curvature $\hat{R}(K)$ is still rather complicated, which is apparent from its superspace expression (A.30e). In the traceless frame, it simplifies dramatically, and using (2.36) and (2.40) one obtains

$$
\begin{aligned}
\hat{R}(K)_{a b c}= & \frac{1}{4} \hat{\nabla}^{d} \hat{R}(M)_{a b c d}-\frac{\mathrm{i}}{24} \hat{R}(Q)^{d e k} \gamma_{a b c} \hat{R}(Q)_{d e k}-\frac{\mathrm{i}}{2} \hat{R}(Q)_{a}{ }^{d k} \gamma_{c} \hat{R}(Q)_{b d k} \\
& +\frac{\mathrm{i}}{30} \chi^{k} \gamma_{c} \hat{R}(Q)_{a b k} .
\end{aligned}
$$


There are actually other component Bianchi identities that we have not analyzed so far. These are differential conditions among the various superconformal component curvatures. In superspace, they are given by the differential equations (A.34). Their component forms can be derived by straightforward component projection.

\subsection{The supersymmetry transformations}

The supersymmetry transformations of the fundamental gauge connections of the Weyl multiplet can be derived directly from the transformations of their corresponding superspace one-forms, using either (A.5) or (A.35). We are mainly interested in their form in the traceless frame, but for comparison with [30] we give the results for arbitrary $\lambda_{2}, \lambda_{3}$ and $\lambda_{5}$, keeping $\lambda_{1}=2$ and $\lambda_{4}=-\frac{1}{2}$ :

$$
\begin{aligned}
\delta e_{m}{ }^{a} & =-\mathrm{i} \xi_{k} \gamma^{a} \psi_{m}{ }^{k} \\
\delta \psi_{m i} & =2 \hat{\mathcal{D}}_{m} \xi_{i}+\frac{1}{12} T^{-a b c} \tilde{\gamma}_{a b c} \gamma_{m} \xi_{i}+2 \mathrm{i} \tilde{\gamma}_{m} \eta_{i}, \\
\delta \mathcal{V}_{m}{ }^{k l} & =-4 \xi^{(k} \hat{\phi}_{m}{ }^{l)}-\frac{16 \mathrm{i}}{15}\left(\lambda_{2}+\frac{5}{8}\right) \xi^{(k} \gamma_{m} \chi^{l)}+4 \psi_{m}{ }^{(k} \eta^{l)}, \\
\delta b_{m} & =\xi_{i} \hat{\phi}_{m}{ }^{i}+\frac{4 \mathrm{i}}{15}\left(\lambda_{2}+\frac{5}{8}\right) \xi_{i} \gamma_{m} \chi^{i}+\psi_{m}{ }^{i} \eta_{i}-2 e_{m}{ }^{a} \lambda_{a} .
\end{aligned}
$$

Here we have restricted to the $Q, S$, and $K$ transformations. In the same way, one can derive the transformations $\delta \hat{\omega}_{m}^{c d}, \delta \hat{\phi}_{\alpha}^{i}$ and $\delta \hat{\mathfrak{f}}_{m a}$, which we omit. For the covariant fields, the transformations are directly given by $(2.7)$, which leads to

$$
\begin{aligned}
\delta T_{a b c}^{-} & =-\frac{\mathrm{i}}{8} \xi^{k} \gamma^{d e} \gamma_{a b c} \hat{R}(Q)_{d e k}-\frac{\mathrm{i}}{5}\left(\lambda_{2}+\frac{25}{24}\right) \xi_{k} \gamma_{a b c} \chi^{k}, \\
\delta \chi^{i} & =\frac{1}{2} D \xi^{i}-\frac{3}{4} \hat{R}(J)_{a b}{ }^{i j} \tilde{\gamma}^{a b} \xi_{j}+\frac{1}{4} \hat{\nabla}_{a} T_{b c d}^{-} \tilde{\gamma}^{b c d} \gamma^{a} \xi^{i}-\mathrm{i} T_{a b c}^{-} \tilde{\gamma}^{a b c} \eta^{i}, \\
\delta D & =-2 \mathrm{i} \xi_{j} \hat{\nabla} \chi^{j}-4 \chi^{k} \eta_{k} .
\end{aligned}
$$

Upon making the appropriate choice of parameters $\lambda_{i}$, these transformations match those of Bergshoeff et al. [30] up to differences in notation and conventions given in appendix B.

\section{The supersymmetric $C^{3}$ invariant}

In [9] it was shown that there were two invariants for conformal supergravity, containing $C^{3}$ and $C \square C$ terms at the component level, where $C$ schematically represents the Weyl tensor. Moreover, the construction of each of these invariants employed the use of two different action principles formulated in terms of superforms. Each of these action principles was built out of a constrained primary superfield, which, when further chosen to be composed of the super-Weyl tensor and its covariant derivatives, yields the two conformal supergravity invariants.

In this section, we will focus on one of these action principles, which we will call the $A$ action principle. It was first constructed using superforms in [44]; see [9] for its construction in conformal superspace. Here we will present its component form as a general density 
formula built upon a certain composite multiplet. Afterwards, we will give the specific choice for the multiplet that generates the $C^{3}$ invariant.

\subsection{The $A$ action principle}

The $A$ action principle is based on a primary dimension $9 / 2$ superfield $A_{\alpha}{ }^{i j k}=A_{\alpha}{ }^{(i j k)}$ obeying the reality condition $\overline{A_{\alpha}{ }^{i j k}}=A_{\alpha i j k}$ and satisfying the differential constraintconstraint $^{9}$

$$
\left.\nabla_{(\alpha}^{(i} A_{\beta}\right)^{j k l)}=0
$$

While a superfield obeying this constraint cannot itself be directly identified as a Lagrangian for some subspace of the full $(1,0)$ superspace, it does possess an important geometric significance. Within the context of the $6 \mathrm{D}$ abelian tensor hierarchy, the multiplet generated by $A_{\alpha}{ }^{i j k}$ provides the minimal version of a five-form gauge multiplet, whose six-form field strength obstructs the closure of the linear multiplet's five-form field strength [44]. The same observation explains why it also appears naturally as the anomaly current multiplet for 6D gauge theories (see e.g. [45] for a recent discussion), as the anomalous current is encoded in a linear multiplet.

In accordance with the superform approach to the construction of supersymmetric invariants [46-49] and following very similar cases in four dimensions [50, 51], it was proposed in [44] to use a closed six-form $J$ built out of the superfield $A_{\alpha}{ }^{i j k}$ as an action principle. The original action principle was given in the context of SU(2) superspace [26] and later extended to conformal superspace [9]. In this latter form, it can be straightforwardly reduced to components and written

$$
I=\int \mathrm{d}^{6} x e \mathcal{L}, \quad e=\operatorname{det}\left(e_{m}{ }^{a}\right)
$$

where $\mathcal{L}$ is a scalar Lagrangian constructed as $\mathcal{L}=\frac{1}{6 !} \varepsilon^{\text {mnpqrs }} J_{\text {mnpqrs }}$, that is as the Hodge dual of the six-form $J$ restricted to spacetime. It is explicitly given by

$$
\begin{aligned}
\mathcal{L}= & F-\frac{\mathrm{i}}{4}\left(\psi_{a i} \Omega^{\prime a i}\right)-\frac{\mathrm{i}}{144}\left(\psi_{d i} \gamma^{d e} \gamma^{a b c} \psi_{e j}\right) S_{a b c}^{+i j} \\
& -\frac{\mathrm{i}}{12}\left(\psi_{a i} \gamma^{a b c} \psi_{b j}\right) E_{c}^{i j}+\frac{1}{16}\left(\psi_{a i} \gamma^{a b c} \psi_{b j}\right)\left(\psi_{c k} A^{i j k}\right) .
\end{aligned}
$$

Here we have introduced the following component fields of $A_{\alpha}{ }^{i j k}: 10$

$$
\begin{aligned}
S_{a b c}^{+i j} & :=\frac{3}{32}\left(\tilde{\gamma}_{a b c}\right)^{\alpha \beta} \nabla_{\alpha k} A_{\beta}{ }^{i j k}\left|, \quad E_{a}^{i j}:=\frac{3}{16}\left(\tilde{\gamma}_{a}\right)^{\alpha \beta} \nabla_{\alpha k} A_{\beta}{ }^{i j k}\right|, \\
\Omega_{a \alpha}^{\prime}{ }^{i} & :=\frac{\mathrm{i}}{32}\left(\tilde{\gamma}_{a}\right)^{\beta \gamma}\left(\nabla_{\beta j} \nabla_{\gamma k} A_{\alpha}{ }^{i j k}\left|+\nabla_{\alpha j} \nabla_{\beta k} A_{\gamma}^{i j k}\right|\right), \\
F & :=\frac{\mathrm{i}}{2^{4} 4 !} \varepsilon^{\alpha \beta \gamma \delta} \nabla_{\alpha i} \nabla_{\beta j} \nabla_{\gamma k} A_{\delta}{ }^{i j k} \mid .
\end{aligned}
$$

\footnotetext{
${ }^{9}$ The names " $A$ action principle" and " $B$ action principle" (discussed later) have no correlation with type A or type B anomalies.

${ }^{10}$ Here we introduce $\Omega_{a \alpha}^{\prime}{ }^{i}$ which differs from $\Omega_{a \alpha}{ }^{i}$ used in [9].
} 
When it is clear from context, we will use the same symbol $A_{\alpha}^{i j k}$ for both the superfield and its lowest component.

The invariance of this general density formula under special conformal transformations is obvious once one verifies that the fields $S_{a b c}^{+{ }^{i j}}, E_{a}{ }^{i j}, \Omega_{a \alpha}^{\prime}{ }^{i}$ and $F$ are each annihilated by $K^{a}$. Invariance under $S$-supersymmetry can be proven by using the $S$-transformation of the gravitino (2.45b) together with $S$-transformations of the components of $A_{\alpha}{ }^{i j k}$ :

$$
\begin{aligned}
\delta_{S} A_{\alpha}^{i j k} & =0, \quad \delta_{S} S_{a b c}^{+i j}=3 \eta_{k} \tilde{\gamma}_{a b c} A^{i j k}, \quad \delta_{S} E_{a}^{i j}=\frac{9}{2} \eta_{k} \tilde{\gamma}_{a} A^{i j k}, \\
\delta_{S} \Omega_{a \alpha}^{\prime i} & =-\frac{8 \mathrm{i}}{3}\left(\gamma_{a b} \eta_{j}\right)_{\alpha} E^{b i j}+\mathrm{i}\left(\gamma^{b c} \eta_{j}\right)_{\alpha} S_{a b c}^{+i j}, \\
\delta_{S} F & =\eta_{k} \tilde{\gamma}^{a} \Omega_{a}^{\prime k} .
\end{aligned}
$$

These transformation laws follow from the definitions of the component fields (3.3).

The invariance of the $A$ action principle under $Q$-supersymmetry follows from its description as a closed superform. Demonstrating closure of the superform is equivalent (although generally more efficient) than demonstrating invariance under $Q$-supersymmetry directly from the spacetime Lagrangian. Let us briefly sketch how the latter procedure goes. The field $A_{\alpha}{ }^{i j k}$ transforms under supersymmetry as

$$
\delta_{Q} A_{\alpha}^{i j k}=-\left(\gamma^{a} \xi_{l}\right)_{\alpha} A_{a}^{i j k l}-\frac{1}{6}\left(\gamma^{a b c} \xi^{(i}\right)_{\alpha} S_{a b c}^{+j k)}+\left(\gamma^{a} \xi^{(i}\right)_{\alpha} E_{a}^{j k)}
$$

The constraint (3.1) is encoded in the absence of a term involving $S_{a b c}^{+i j k l}:=$ $\left(\tilde{\gamma}_{a b c}\right)^{\alpha \beta} \nabla_{\alpha}^{(i} A_{\beta}{ }^{j k l)} \mid$ on the right-hand side. Now the term $A_{a}{ }^{i j k l}$ does not appear in the action and so its $\delta_{Q}$ transformation does not need to be computed directly. This can be verified by computing $\delta_{Q}$ of the terms involving $A_{\alpha}{ }^{i j k}$ in the action and integrating by parts any expressions with $\mathcal{D}_{m} \xi_{i}^{\alpha}$ to give a covariant result. The term involving $A_{b}{ }^{i j k l}$ turns out to be multiplied by a $\psi^{3} \xi$ term, and the various contractions of Lorentz and SU(2) indices project this term out. The advantage of the superform method is that this sequence of steps is encoded automatically.

It is clear that the constraint (3.1) is rather weak and describes a multiplet that is rather long, only a few components of which have been mentioned above. Nevertheless, the supersymmetry transformations of all components are dictated by closure of the supersymmetry algebra, making use of the constraint (3.1). For example,

$$
\delta_{Q} A_{a}^{i j k l}=\frac{1}{2} \xi_{p} \gamma_{a} \Xi^{i j k l p}-\xi^{(i} \Xi_{a}^{j k l)}
$$

for some spinor $\Xi_{\alpha}{ }^{i j k l p}$ and some vector-spinor $\Xi_{a \alpha}{ }^{i j k}$. While the multiplet is rather long, only a small number of the component fields appear in the action, so that in practice one only needs some of the supersymmetry transformations. These are given below in the 
traceless frame:

$$
\begin{aligned}
\delta_{Q} E_{a}{ }^{i j}= & \frac{\mathrm{i}}{2} \xi_{k} \gamma^{b} \tilde{\gamma}_{a} \hat{\nabla}_{b} A^{i j k}-\frac{\mathrm{i}}{12} \xi_{k} \gamma_{a} \tilde{\gamma}^{b c d} A^{i j k} T_{b c d}^{-}-\frac{1}{4} \xi_{k} \gamma_{a b} \Xi^{b i j k} \\
& -4 \mathrm{i} \xi^{(i} \Omega_{a}^{\prime j)}-\mathrm{i} \xi^{(i} \gamma_{a} \tilde{\gamma}^{b} \Omega_{b}^{\prime j)} \\
\delta_{Q} S_{a b c}^{+i j}= & \frac{\mathrm{i}}{4} \xi_{k} \gamma^{d} \tilde{\gamma}_{a b c} \hat{\nabla}_{d} A^{i j k}-\frac{1}{8} \xi_{k} \gamma^{d} \tilde{\gamma}_{a b c} \Xi_{d}{ }^{i j k}+\mathrm{i} \xi^{(i} \gamma^{d} \tilde{\gamma}_{a b c} \Omega_{d}^{\prime j)} \\
\delta_{Q} \Omega_{a \alpha}^{\prime i}= & \left(\gamma_{a} \xi^{i}\right)_{\alpha} F+\frac{1}{3}\left(\gamma_{a b c} \xi_{j}\right)_{\alpha} \hat{\nabla}^{b} E^{c i j}+\frac{1}{72}\left(\gamma_{a b} \gamma^{c d e} \xi_{j}-\gamma^{c d e} \tilde{\gamma}_{a b} \xi_{j}\right)_{\alpha} \hat{\nabla}^{b} S_{c d e}^{+i j} \\
& +\left(\gamma^{b} \xi_{j}\right)_{\alpha} C_{b a}{ }^{(i j)}+\left(\gamma_{a b c} \xi^{i}\right)_{\alpha} C^{[b c]}-\frac{3 \mathrm{i}}{5} A_{\alpha}{ }^{i j k}\left(\xi_{j} \gamma_{a} \chi_{k}\right)+\frac{3 \mathrm{i}}{5}\left(\xi_{j} A^{i j k}\right)\left(\gamma_{a} \chi_{k}\right)_{\alpha} \\
& +\frac{1}{288} S_{b c d}^{+i j} T_{e f g}^{-}\left(\gamma_{a} \tilde{\gamma}^{e f g} \gamma^{b c d} \xi_{j}-\gamma^{b c d} \tilde{\gamma}^{e f g} \gamma_{a} \xi_{j}\right)_{\alpha} \\
& +\frac{1}{36} E_{b}{ }^{i j} T_{c d e}^{-}\left(\gamma_{a} \tilde{\gamma}^{c d e} \gamma^{b} \xi_{j}+\gamma^{b} \tilde{\gamma}^{c d e} \gamma_{a} \xi_{j}\right)_{\alpha}, \\
\delta_{Q} F= & -\mathrm{i} \xi_{i} \hat{\nabla}_{a} \Omega^{\prime a i}-\frac{\mathrm{i}}{24} T^{-a b c} \xi_{k} \gamma^{d} \tilde{\gamma}_{a b c} \Omega_{d}^{\prime k}-\frac{\mathrm{i}}{72} \xi_{i} \gamma^{a b} \gamma^{c d e} \hat{R}(Q)_{a b j} S_{c d e}^{+i j}
\end{aligned}
$$

Using the above supersymmetry transformations one can check that the invariant (3.2) is indeed supersymmetric. Note that the transformation of the vector-spinor $\Omega_{a \alpha}^{\prime}{ }^{i}$ involves two additional fields that do not appear in the action: an antisymmetric tensor $C_{[a b]}$ and a rank-two tensor $C_{a b}{ }^{(i j)}$ symmetric in its $\mathrm{SU}(2)$ indices.

We underline here that these supersymmetry transformations are sufficient to prove invariance of the action. They arise as a consequence of closure of the algebra provided the constraint (3.1) holds; therefore, there is no need to analyze the transformation properties of the entire multiplet.

\subsection{The supersymmetric $C^{3}$ invariant in components}

Having elaborated the component structure of the $A$ action principle we now provide an immediate application: the derivation of the supersymmetric $C^{3}$ invariant at the component level. The suitable superfield $A_{\alpha}^{i j k}$ was constructed in [9]. At the component level, it corresponds to ${ }^{11}$

$$
\begin{aligned}
A_{\alpha}{ }^{i j k}= & -\frac{64 \mathrm{i}}{15}\left(\gamma_{a} \chi^{(i}\right)_{\alpha}\left(\frac{4}{45} \chi^{j} \gamma^{a} \chi^{k)}+\hat{R}(Q)_{b c}{ }^{j} \gamma^{a} \hat{R}(Q)^{b c k)}-3 \mathrm{i} \hat{R}(J)_{b c}{ }^{j k)} T^{-a b c}\right) \\
& -128\left(\gamma_{a} \hat{R}(Q)_{b c}{ }^{(i}\right)_{\alpha}\left(\hat{R}(J)_{d}{ }^{c j k)} T^{-a b d}+\frac{2 \mathrm{i}}{3} \hat{R}(Q)^{a d j} \gamma^{c} \hat{R}(Q)_{d}^{b k)}\right) .
\end{aligned}
$$

One may verify that it is $S$-invariant and transforms under supersymmetry so that the constraint (3.1) is satisfied. The remaining composites $S_{a b c}^{+i j}, E_{a}{ }^{i j}, \Omega_{a \alpha}^{\prime}{ }^{i}$ and $F$ appearing in $(3.2 \mathrm{~b})$ can be computed directly from the supersymmetry transformations (3.7) or equivalently from the superspace definitions (3.3). This is a straightforward task but the explicit calculation becomes technically quite involved, so we made use of the computer algebra program Cadabra $[52,53]$. In a separate supplementary file, we give the explicit

\footnotetext{
${ }^{11}$ Relative to [9], we have renormalized the choice for $A_{\alpha}{ }^{i j k}$ by a factor of -64 .
} 
expressions for each of these components. The bosonic part of the Lagrangian is given by

$$
\begin{aligned}
\mathcal{L}= & \frac{8}{3} C_{a b c d} C^{a b e f} C^{c d}{ }_{e f}-\frac{16}{3} C_{a b c d} C^{a e c f} C^{b}{ }_{e}{ }_{f}-2 C_{a b c d} \mathcal{R}^{a b i j} \mathcal{R}^{c d}{ }_{i j}+4 \mathcal{R}_{a b}{ }^{i j} \mathcal{R}^{a c}{ }_{i}{ }^{k} \mathcal{R}^{b}{ }_{c j k} \\
& -\frac{32}{225} D^{3}-\frac{4}{15} D C_{a b c d} C^{a b c d}+\frac{8 \mathrm{i}}{5} D \mathcal{R}_{a b}{ }^{i j} \mathcal{R}^{a b}{ }_{i j}+\frac{128}{15} T_{a b c}^{-} T^{-a d e} D C^{b}{ }_{d}^{c}{ }_{e} \\
& +\frac{64}{15} T_{a b c}^{-} D \hat{\nabla}^{a} \hat{\nabla}_{d} T^{-b c d}-\frac{4}{5} D \hat{\mathcal{D}}^{a} T_{a b c}^{-} \hat{\mathcal{D}}_{d} T^{-b c d}+\frac{4}{15} D \hat{\mathcal{D}}_{a} T_{b c d}^{-} \hat{\mathcal{D}}^{a} T^{-b c d} \\
& -\frac{4}{3} D \hat{\mathcal{D}}_{a} T_{b c d}^{-} \hat{\mathcal{D}}^{b} T^{-a c d}-\frac{16}{5} T_{a b c}^{-} T^{-a b d} T^{-c e f} T_{d e f}^{-}{ }^{D} \\
& -\frac{32}{3} T_{a b c}^{-} C^{a b d e} \hat{\mathcal{D}}^{f} C^{c}{ }_{d e f}+\frac{16}{3} C_{a b c d} C^{a b e f} \hat{\mathcal{D}}^{c} T^{-d}{ }_{e f} \\
& -16 T_{a b c}^{-} \hat{\mathcal{D}}_{d} T^{-a b e} \hat{\nabla}_{e} \hat{\nabla}_{f} T^{-c d f}-16 T_{a b c}^{-} \hat{\mathcal{D}}_{d} T^{-a d e} \hat{\nabla}_{e} \hat{\nabla}_{f} T^{-b c f} \\
& -48 T_{a b c}^{-} \hat{\mathcal{D}}_{d} T^{-a d e} \hat{\nabla}^{b} \hat{\nabla}^{f} T^{-c}{ }_{e f}+16 \hat{\mathcal{D}}^{e} T_{e a b}^{-} \hat{\mathcal{D}}^{f} T_{f c d}^{-} \hat{\mathcal{D}}^{a} T^{-b c d} \\
& -40 T_{a b e}^{-} T^{-c d e} \hat{\mathcal{D}}_{f} T^{-f a b} \hat{\mathcal{D}}^{g} T_{g c d}^{-}+16 T_{a b c}^{-} C^{a b d e} \hat{\nabla}^{c} \hat{\nabla}^{f} T_{d e f}^{-} \\
& -16 T_{a b c}^{-} C^{a b d e} \hat{\nabla}_{d} \hat{\nabla}^{f} T^{-c}{ }_{e f}-4 C_{a b c d} \hat{\mathcal{D}}_{e} T^{-a b e} \hat{\mathcal{D}}_{f} T^{-c d f} \\
& +8 C_{a b c d} \hat{\mathcal{D}}_{e} T^{-a b f} \hat{\mathcal{D}}_{f} T^{-c d e}-\frac{64}{3} T^{-f b}{ }_{d} \hat{\mathcal{D}}^{e} C_{e a b c} \hat{\mathcal{D}}_{f} T^{-a c d} \\
& +32 T^{-a b}{ }_{d} \hat{\mathcal{D}}^{e} C_{e a b c} \hat{\mathcal{D}}_{f} T^{-f c d}-32 T_{f g c}^{-} T^{-f g d} \hat{\mathcal{D}}^{c} T_{d a b}^{-} \hat{\mathcal{D}}_{e} T^{-e a b} \\
& -8 \hat{\mathcal{D}}_{e} T_{b a d}^{-} \hat{\mathcal{D}}^{e} T^{-c a d} T^{-f g b} T_{f g c}^{-}-8 T_{a b c}^{-} T^{-a b d} C^{c e f g} \hat{\mathcal{D}}_{e} T_{d f g}^{-} \\
& -\frac{8}{3} T_{a b c}^{-} \mathcal{R}^{a b}{ }^{i j} \hat{\mathcal{D}}_{d} \mathcal{R}^{c d}{ }_{i j}+\frac{28}{3} T_{a b c}^{-} \mathcal{R}^{a d}{ }_{i j} \hat{\mathcal{D}}_{d} \mathcal{R}^{b c}{ }_{i j}-\frac{32}{9} \mathcal{R}_{a b}{ }_{i j} \mathcal{R}_{c d i j} \hat{\mathcal{D}}^{a} T^{-b c d} \\
& +4 T_{e f b}^{-} T^{-e f c} T_{g h a}^{-} T^{-g h}{ }_{c} \hat{\mathcal{D}}_{d} T^{-d a b}-8 T_{a b c}^{-} T^{-a b d} T_{e f g}^{-} T^{-e f h} C^{c g}{ }_{d h} \\
& +12 T_{a b c}^{-} T^{-a d e} \mathcal{R}^{b c i j} \mathcal{R}_{d e}{ }_{i j}+\text { fermion terms } .
\end{aligned}
$$

Note that in the previous result there is some hidden dependence on the special conformal connection $\hat{\mathfrak{f}}_{a b}$, which can be made explicit by using

$$
\begin{aligned}
\hat{\nabla}_{a} \hat{\nabla}_{b} T_{c d e}^{-} & =\hat{\mathcal{D}}_{a} \hat{\mathcal{D}}_{b} T_{c d e}^{-}-\hat{\mathfrak{f}}_{a f}\left[K^{f}, \hat{\nabla}_{b}\right] T_{c d e}^{-}+\text {fermion terms } \\
& =\hat{\mathcal{D}}_{a} \hat{\mathcal{D}}_{b} T_{c d e}^{-}-2 \hat{\mathfrak{f}}_{a b} T_{c d e}^{-}-6 \hat{\mathfrak{f}}_{a[c} T_{d e] b}^{-}+6 \hat{\mathfrak{f}}_{a}^{f} T_{f[c d}^{-} \eta_{e] b}+\text { fermion terms }
\end{aligned}
$$

and the results in section 2 .

We conclude this section by mentioning one useful consistency check of the previous result. By computing the variation of the action with respect to the $D$ field one should obtain the component projection of the supercurrent for the supersymmetric $C^{3}$ invariant. Upon doing this, one finds a supercurrent in the two parameter family described in [9] with $c_{1}=0$.

\section{The supersymmetric $C \square C$ invariant}

Another conformal supergravity invariant was constructed in [9], corresponding to the supersymmetrization of $C_{a b c d} \square C^{a b c d}$. It makes use of a different superform based on another constrained primary superfield. Curiously, this construction, which we denote the $B$ action principle, is not based on an $S$-invariant superform, which leads to explicit dependence on 
the $S$-supersymmetry and special conformal connections in the component action. As discussed in [9], another choice for the basic primary superfield leads to the supersymmetric $F \square F$ invariant minimally coupled to conformal supergravity. Both results are given below.

\subsection{The $B$ action principle}

Let us begin by describing the $B$ action principle in components. Its building block is a primary dimension 3 superfield $B_{a}{ }^{i j}=B_{a}{ }^{(i j)}$, which is pseudoreal, $\overline{B_{a}{ }^{i j}}=B_{a i j}$, and satisfies the differential constraint

$$
\nabla_{\alpha}^{(i} B^{\beta \gamma j k)}=-\frac{2}{3} \delta_{\alpha}^{[\beta} \nabla_{\delta}^{(i} B^{\gamma] \delta j k)} .
$$

Its corresponding superform action principle was given in [9]. Its reduction to components, following (3.2a), is straightforward and leads to

$$
\begin{aligned}
\mathcal{L}= & F^{\prime}+\left(\psi_{m i} \gamma^{m} \Omega^{i}\right)+\frac{\mathrm{i}}{12}\left(\psi_{a i} \gamma^{a b c} \psi_{b j}\right) E_{c}{ }^{i j}+\frac{1}{16}\left(\psi_{m i} \gamma^{m n p} \psi_{n j}\right)\left(\psi_{p k} \rho^{i j k}\right) \\
& -16 \mathfrak{f}^{a b} C_{a b}-8 \mathrm{i}\left(\psi_{m i} \gamma^{m n} \Lambda_{a}^{i}\right) \mathfrak{f}_{n}{ }^{a}+8 \mathrm{i}\left(\psi_{m i} \gamma^{m n p} \psi_{n j}\right) \mathfrak{f}_{p}{ }^{a} B_{a}{ }^{i j} \\
& +2\left(\phi_{a j} \rho^{a j}\right)+\frac{1}{3}\left(\psi_{m j} \gamma^{m n} \gamma^{a} \tilde{\gamma}^{b} \phi_{n k}\right) C_{a b}{ }^{j k}+\frac{3 \mathrm{i}}{2}\left(\psi_{m i} \gamma^{m n p} \psi_{n j}\right)\left(\phi_{p k} \Lambda^{i j k}\right) .
\end{aligned}
$$

The various component fields are defined by successive application of superspace spinor derivatives,

$$
\begin{aligned}
\Lambda^{\alpha i j k} & :=\frac{\mathrm{i}}{3} \nabla_{\beta}^{(i} B^{\beta \alpha j k)} \mid, & \Lambda_{\alpha b}{ }^{i} & :=\frac{2 \mathrm{i}}{3} \nabla_{\alpha j} B_{b}{ }^{i j} \mid, \\
C^{i j k l} & :=\frac{1}{4} \nabla_{\alpha}^{(i} \Lambda^{\alpha j k l)} \mid, & C_{a b}{ }^{i j} & :=\frac{1}{8}\left(3\left(\gamma_{a b}\right)_{\beta}{ }^{\alpha}+\eta_{a b} \delta_{\beta}{ }^{\alpha}\right) \nabla_{\alpha k} \Lambda^{\beta i j k} \mid, \\
C_{a b} & :=\frac{1}{8}\left(\tilde{\gamma}_{a}\right)^{\alpha \beta} \nabla_{\alpha k} \Lambda_{\beta b}{ }^{k} \mid, & & \\
\rho_{\alpha}{ }^{i j k} & :=-\frac{4 \mathrm{i}}{5} \nabla_{\alpha l} C^{i j k l} \mid, & \rho_{a}{ }^{\gamma i} & :=-\frac{\mathrm{i}}{6}\left(\tilde{\gamma}_{a}\right)^{\alpha \beta} \nabla_{\alpha j} C_{\beta}{ }^{\gamma i j} \mid, \\
E_{a}{ }^{i j} & :=\frac{3}{16}\left(\tilde{\gamma}_{a}\right)^{\alpha \beta} \nabla_{\alpha k} \rho_{\beta}{ }^{i j k} \mid, & & \\
\Omega^{\alpha i} & :=\frac{\mathrm{i}}{18} \nabla_{\beta j} E^{\beta \alpha}{ }^{\beta i j} \mid, & F & =\frac{1}{8} \nabla_{\alpha j} \Omega^{\alpha j} \mid,
\end{aligned}
$$

where we abuse notation somewhat by denoting e.g. $\Lambda^{\alpha i j k}$ both as a superfield and as its lowest component. In the Lagrangian (4.2), we have written $F^{\prime}$ and $\Omega^{\prime \alpha i}$ for the terms involving zero and one gravitini, respectively. These are given in terms of $F$ and $\Omega^{\alpha i}$ as

$$
\begin{aligned}
\Omega^{\prime \alpha i} & =\Omega^{\alpha i}+\frac{32}{3} B_{a}{ }^{i j} \nabla_{b} R(Q)^{a b \alpha}{ }_{j}+\frac{3}{4} \Lambda^{\beta i j k}\left(\gamma^{a b}\right)_{\beta}{ }^{\alpha} R(J)_{a b j k}, \\
F^{\prime} & =F-\frac{16 \mathrm{i}}{3} \Lambda_{\alpha b}{ }^{i} \nabla_{c} R(Q)^{b c \alpha}{ }_{i}+4 B_{a i j} \nabla_{b} R(J)^{a b i j}+\frac{2}{3} C_{a b i j} R(J)^{a b i j},
\end{aligned}
$$

where we have written the vector derivative and gravitino curvature in the original frame to maintain contact with [9].

As already alluded to, the connections $\mathfrak{f}_{m}{ }^{a}$ and $\phi_{m \alpha}{ }^{i}$ appear explicitly within the Lagrangian (4.2), so invariance under both special conformal and $S$-supersymmetry transformations holds only up to total derivatives. It does not seem possible to eliminate this 
explicit dependence in the density formula, although we will find that at least for the bosonic action, we can eliminate most of the explicit $\mathfrak{f}_{m}{ }^{a}$ connections by judiciously adding a certain total derivative.

As we are primarily working with the traceless connections when in components, it is useful to give the density formula in that case as well. Its form is slightly modified,

$$
\begin{aligned}
\mathcal{L}= & \widehat{F}+\left(\psi_{a i} \widehat{\Omega}^{a i}\right)+\frac{\mathrm{i}}{12}\left(\psi_{a i} \gamma^{a b c} \psi_{b j}\right) \widehat{E}_{c}^{i j}+\frac{1}{16}\left(\psi_{m i} \gamma^{m n p} \psi_{n j}\right)\left(\psi_{p k} \rho^{i j k}\right) \\
& -16 \hat{\mathfrak{f}}^{a b} C_{a b}-8 \mathrm{i}\left(\psi_{m i} \gamma^{m n} \Lambda_{a}^{i}\right) \hat{\mathfrak{f}}_{n}{ }^{a}+8 \mathrm{i}\left(\psi_{m i} \gamma^{m n p} \psi_{n j}\right) \hat{\mathfrak{f}}_{p}{ }^{a} B_{a}{ }^{i j} \\
& +2\left(\hat{\phi}_{a j} \rho^{a j}\right)+\frac{1}{3}\left(\psi_{m j} \gamma^{m n} \gamma^{a} \tilde{\gamma}^{b} \hat{\phi}_{n k}\right) C_{a b}{ }^{j k}+\frac{3 \mathrm{i}}{2}\left(\psi_{m i} \gamma^{m n p} \psi_{n j}\right)\left(\hat{\phi}_{p k} \Lambda^{i j k}\right),
\end{aligned}
$$

with the hatted fields defined by

$$
\begin{aligned}
\widehat{F} & =F^{\prime}+2 C^{a b}\left(\frac{2}{15} \eta_{a b} D-2 \nabla^{c} T_{c a b}^{-}+T_{a}^{-c d} T_{b c d}^{-}\right)+\frac{\mathrm{i}}{5}\left(\chi_{j} \gamma_{a} \rho^{a j}\right) \\
\widehat{\Omega}_{a}{ }^{i} & =\gamma_{a} \Omega^{\prime i}+\mathrm{i} \gamma_{a b} \Lambda_{c}^{i}\left(\frac{2}{15} \eta^{b c} D-2 \nabla_{d} T^{-d b c}+T^{-b d e} T_{d e}^{-c}\right)-\frac{\mathrm{i}}{30} \gamma_{a b} \gamma_{c} \tilde{\gamma}_{d} \gamma^{b} \chi_{j} C^{c d i j} \\
\widehat{E}_{a}^{i j} & =E_{a}^{i j}-12 B^{b i j}\left(\frac{2}{15} \eta_{a b} D-2 \nabla^{c} T_{c a b}^{-}+T_{a}^{-c d} T_{b c d}^{-}\right)+\frac{9 \mathrm{i}}{5}\left(\chi_{k} \gamma_{a} \Lambda^{i j k}\right)
\end{aligned}
$$

The explicit supersymmetry relations between the various component fields in this action principle are quite complicated and not very enlightening, so we do not give them here.

For the actions that we will be considering, we will mainly be interested in their bosonic Lagrangians, and these amount to

$$
\mathcal{L}=\widehat{F}-16 \hat{\mathfrak{f}}^{a b} C_{a b}
$$

It turns out that at least for the bosonic parts, it is possible to eliminate the explicit appearance of the $K$-connection within the bosonic action and render a $K$-invariant result. Adding a total derivative

$$
\mathcal{L}_{\text {add }}=-\hat{\nabla}_{a}\left(4 \hat{\nabla}_{b} C^{(a b)}-\hat{\nabla}^{a} C_{c}^{c}\right)+16 \hat{\mathfrak{f}}_{a b} C^{(a b)}
$$

has the effect of removing the symmetric part of $C_{a b}$ from the $K$-connection term, giving

$$
\mathcal{L}^{\prime}=\mathcal{L}+\mathcal{L}_{\text {add }}=\widehat{F}-\hat{\nabla}_{a}\left(4 \hat{\nabla}_{b} C^{(a b)}-\hat{\nabla}^{a} C_{c}{ }^{c}\right)-16 \hat{\mathfrak{f}}_{[a b]} C^{[a b]}
$$

Henceforth, we will drop the prime on the Lagrangian.

The advantage of this modification is that the antisymmetric (and bosonic) part of $\hat{\mathfrak{f}}_{a b}$ is just the curl of the dilatation gauge field $b_{m}$. In the circumstances we will be interested in, $C^{[a b]}$ will be divergenceless and so this term can be dropped. Equivalently, the $K$ transformation of the other terms in (4.9) is proportional to $\hat{\nabla}^{a} C_{[a b]}$, and so becomes $K$ invariant when this quantity vanishes; therefore, the explicit $K$-connection must also vanish up to a total derivative. This is the case when $B_{a}{ }^{i j}$ describes a four-form field strength 
multiplet (with $C_{[a b]}$ the Hodge dual of the supercovariant field strength) as studied e.g. in [44]. There, in addition to the constraint (4.1), the authors found that

$$
C_{a}{ }^{a i j}=-4 \hat{\nabla}_{a} B^{a i j}
$$

Both the models we construct in this paper using the $B$-action principle will actually satisfy this extra condition. It is possible that for this restricted class, the $B$-action principle might be significantly simplified by eliminating a number of the $K$ and $S$ connections, but it seems impossible to remove all of them simultaneously, so we have not attempted to massage its form any further.

\subsection{The supersymmetric $C \square C$ invariant in components}

Using the $B$ action principle, one can directly construct the $C \square C$ invariant. The appropriate superfield $B_{a}{ }^{i j}$ should be a composite built out of the standard Weyl multiplet fields and was given in superspace (up to a change in normalization) as [9]

$$
B^{\alpha \beta i j}=-4 W^{\gamma[\alpha} Y_{\gamma}^{\beta] i j}-32 \mathrm{i} X_{\gamma}{ }^{\alpha \delta(i} X_{\delta}^{\beta \gamma j)}+10 \mathrm{i} X^{\alpha(i} X^{\beta j)}
$$

Its lowest component corresponds to

$$
B_{a}^{i j}=T_{a b c}^{-} R(J)^{b c i j}+\mathrm{i}\left(\hat{R}(Q)_{b c}{ }^{i} \gamma_{a} \hat{R}(Q)^{b c j}\right)+\frac{2 \mathrm{i}}{45}\left(\chi^{i} \gamma_{a} \chi^{j}\right)
$$

The Lagrangian can then be built out of successive applications of superspace spinor derivatives or, equivalently, supersymmetry transformations to give the various composite objects. These are quite intricate and we made use of the computer algebra program Cadabra to help in their construction. Their full expressions are given in a supplementary file. Right now, an important feature is that (4.10) holds, and so this describes a four-form field strength multiplet. Indeed, the bosonic part of $C_{[a b]}$ can be written

$$
\begin{aligned}
C_{[a b]}= & \frac{1}{8} \varepsilon_{a b}{ }^{c d e f}\left(\hat{R}(M)_{c d}^{g h} \hat{R}(M)_{e f g h}-\hat{R}(J)_{c d}^{i j} \hat{R}(J)_{e f i j}\right)+\hat{\nabla}^{c} B_{a b c}, \\
B_{a b c}= & \left.-\frac{4}{15} D T_{a b c}^{-}-\frac{3}{2} T_{[a}^{-d e} \hat{R}(M)_{b c] d e}+3 T_{d e[a}^{-} \hat{R}(M)_{b}{ }^{d e}{ }_{c]}\right] \\
& +6 T_{d e[a}^{-} \hat{\nabla}^{d} T_{b c]}^{-e}-4 T_{a d}^{-e} T_{b e}^{-f} T_{c f}^{-d},
\end{aligned}
$$

and so the bosonic Lagrangian can be compactly written as (4.9) without the final term.

Even with all these manipulations, the bosonic Lagrangian is fairly involved and it turns out to be useful to perform yet another set of integrations by parts to place it into a 
final form that will be useful later on. Up to a total derivative, we find

$$
\begin{aligned}
\mathcal{L}= & \frac{1}{3} C_{a b c d} \hat{\nabla}^{2} C^{a b c d}-\frac{1}{3} C_{a b}{ }^{c d} C_{c d}{ }^{e f} C_{e f}{ }^{a b}-\frac{4}{3} C_{a b c d} C^{a e c f} C^{b}{ }_{e}{ }_{f} \\
& -\mathcal{R}_{a b}{ }^{i j} \hat{\nabla}^{2} \mathcal{R}^{a b}{ }_{i j}-2 \mathcal{R}_{a}{ }^{b}{ }_{i}{ }^{j} \mathcal{R}^{a}{ }_{c j}{ }^{k} \mathcal{R}_{b}{ }^{c}{ }_{k}{ }^{i}+2 C^{a b c d} \mathcal{R}_{a b}{ }^{i j} \mathcal{R}_{c d} i j \\
& +\hat{\mathfrak{f}}_{a}{ }^{b}\left(\frac{32}{3} C^{a c d e} C_{b c d e}-8 \mathcal{R}_{b c}{ }^{i j} \mathcal{R}^{a c}{ }_{i j}\right)-4 \hat{\mathfrak{f}}_{a}{ }^{a}\left(C_{b c d e} C^{b c d e}-\mathcal{R}_{b c}{ }^{i j} \mathcal{R}^{b c}{ }_{i j}\right) \\
& +\frac{4}{45} D \hat{\nabla}^{2} D+\frac{8}{225} D^{3}+\frac{2}{15} D C_{a b c d} C^{a b c d}-\frac{14}{15} D \mathcal{R}_{a b}{ }^{i j} \mathcal{R}^{a b}{ }_{i j} \\
& +\frac{20}{3} T^{-a b e} C_{a b}{ }^{c d} \hat{\nabla}^{f} C_{f e c d}+4 T^{-a b e} \hat{\nabla}^{f} C_{a b}{ }^{c d} C_{f e c d} \\
& +2 T_{a b c}^{-} \hat{\nabla}_{d} \mathcal{R}^{a b i j} \mathcal{R}^{c d}{ }_{i j}+4 T_{a b c}^{-} \hat{\nabla}_{d} \mathcal{R}^{a d}{ }_{i j} \mathcal{R}^{b c}{ }_{i j} \\
& -4 T_{a b c}^{-} \Delta^{4} T^{-a b c}-\frac{16}{3} C_{a b c d} T^{-a b e} \hat{\nabla}_{e} \hat{\nabla}_{f} T^{-c d f}-\frac{8}{3} C_{a b c d} T^{-a b e} \hat{\nabla}_{f} \hat{\nabla}_{e} T^{-c d f} \\
& +\frac{16}{3} C_{a b}{ }^{c d} T^{-a e f} \hat{\nabla}^{b} \hat{\nabla}_{c} T_{d e f}^{-}-4 C_{a b}{ }^{c d} \hat{\nabla}^{a} T^{-b e f} \hat{\nabla}_{c} T_{d e f}^{-}-6 C_{a b}{ }^{c d} \hat{\nabla}_{e} T^{-a b f} \hat{\nabla}_{f} T^{-c d e} \\
& -\frac{16}{15} D T_{a b c}^{-} \hat{\nabla}^{a} \hat{\nabla}_{d} T^{-b c d}+\frac{8}{15} D \hat{\nabla}^{a} T_{a b c}^{-} \hat{\nabla}_{d} T^{-b c d} \\
& -2 T_{a b c}^{-} T^{-a d e} \mathcal{R}^{b c}{ }_{i j} \mathcal{R}_{d e}{ }^{i j}-\frac{4}{3} C_{a b e f} C^{c d e f} T_{a b g}^{-} T^{-c d g} \\
& -\frac{1}{2} \hat{\nabla}^{a_{1}} T_{a_{1} a b}^{-} \hat{\nabla}^{a_{2}} T_{a_{2} c d}^{-} \hat{\nabla}^{a_{3}} T_{a_{3} e f}^{-} \varepsilon^{a b c d e f}-6 T_{a b}^{-g} \hat{\nabla}^{a_{1}} T_{a_{1} g c}^{-} \hat{\nabla}_{d} \hat{\nabla}^{a_{2}} T_{a_{2} e f}^{-} \varepsilon^{a b c d e f} \\
& +8 C_{a b c d} T^{-e c d} T_{e f g}^{-} \hat{\nabla}^{a} T^{-b f g}+\frac{10}{3} T_{a b c}^{-} T^{-a e d} T^{-b f}{ }_{d} \hat{\nabla}^{2} T^{-c}{ }_{e f} \\
& -2 T_{a b c}^{-} T^{-a b d} \hat{\nabla}^{c} T_{d e f}^{-} \hat{\nabla}_{g} T^{-e f g}+4 T_{a b c}^{-} T^{-a}{ }_{d e} \hat{\nabla}^{f} T^{-b d g} \hat{\nabla}_{f} T^{-c e}{ }_{g} \\
& +2 C_{a b c d} T^{-a b e} T^{-c f g} T^{-d}{ }_{f h} T_{e g}^{-h}+\frac{8}{15} D T_{a b c}^{-} T^{-a b d} T^{-c e f} T_{d e f}^{-}+\text {fermion terms }
\end{aligned}
$$

where we have introduced the $K$-invariant fourth order operator

$T^{-a b c} \Delta^{4} T_{a b c}^{-}:=T^{-a b c}\left(\hat{\nabla}_{a} \hat{\nabla}^{d} \hat{\nabla}^{2} T_{b c d}^{-}+\hat{\nabla}^{2} \hat{\nabla}_{a} \hat{\nabla}^{d} T_{b c d}^{-}+\frac{1}{3} \hat{\nabla}_{a} \hat{\nabla}^{2} \hat{\nabla}^{d} T_{b c d}^{-}-\frac{4}{3} \hat{\nabla}_{e} \hat{\nabla}_{a} \hat{\nabla}^{d} \hat{\nabla}^{e} T_{b c d}^{-}\right)$.

In order to extract the $K$-connections, which encode the Ricci tensor contributions, it is useful to have the following results, which hold up to fermionic terms:

$$
\begin{aligned}
\hat{\nabla}_{a} \hat{\nabla}_{b} D= & \hat{\mathcal{D}}_{a} \hat{\mathcal{D}}_{b} D-4 \hat{f}_{a b} D \\
\hat{\nabla}_{a} \hat{\nabla}_{b} \mathcal{R}_{c d}{ }^{i j}= & \hat{\mathcal{D}}_{a} \hat{\mathcal{D}}_{b} \mathcal{R}_{c d}{ }^{i j}-4 \hat{f}_{a b} \mathcal{R}_{c d}{ }^{i j}+4 \hat{f}_{a[c} \mathcal{R}_{d] b}{ }^{i j}-4 \hat{f}_{a}^{e} \mathcal{R}_{e[c}{ }^{i j} \eta_{d] b} \\
\hat{\nabla}_{a} \hat{\nabla}_{b} C_{c d e f}= & \hat{\mathcal{D}}_{a} \hat{\mathcal{D}}_{b} C_{c d e f}-4 \hat{f}_{a b} C_{c d e f}+4 \hat{f}_{a[c} C_{d] b e f}-4 \hat{f}_{a}^{g} \eta_{b[c} C_{d] g e f} \\
& +4 \hat{f}_{a[e} C_{f] b c d}-4 \hat{f}_{a}^{g} \eta_{b[e} C_{f] g c d} \\
\hat{\nabla}_{a} \hat{\nabla}_{b} \hat{\nabla}_{c} T^{-d e f}= & \hat{\mathcal{D}}_{a} \hat{\nabla}_{b} \hat{\nabla}_{c} T^{-d e f}-8 \hat{f}_{a(b} \hat{\mathcal{D}}_{c)} T^{-d e f}+2 \eta_{b c} \hat{f}_{a}^{g} \hat{\mathcal{D}}_{g} T^{-d e f} \\
& -12 \hat{f}_{a}^{[d} \hat{\mathcal{D}}_{(b} T_{c)}^{-e f]}+12 \hat{f}_{a}^{g} \delta_{(b}^{[d} \hat{\mathcal{D}}_{c)} T_{g}^{-e f]} \\
\hat{\nabla}_{a} \hat{\nabla}_{b} \hat{\nabla}^{c} \hat{\nabla}_{c} T^{-d e f}= & \hat{\mathcal{D}}_{a} \hat{\nabla}_{b} \hat{\nabla}^{c} \hat{\nabla}_{c} T^{-d e f}-6 \hat{f}_{a b} \hat{\nabla}^{c} \hat{\nabla}_{c} T^{-d e f}-6 \hat{\nabla}^{c} \hat{\nabla}_{c} T_{b}^{-[d e} \hat{f}_{a}^{f]} \\
& +6 \hat{f}_{a}{ }^{g} \hat{\nabla}^{c} \hat{\nabla}_{c} T_{g}^{-[d e} \delta_{b}^{f]}+4 \hat{f}_{a}{ }^{c} \hat{\nabla}_{b} \hat{\nabla}_{c} T^{-d e f}-12 \hat{f}_{a}{ }^{[d} \hat{\nabla}_{b} \hat{\nabla}_{c} T^{-e f] c} \\
& +12 \hat{f}_{a c} \hat{\nabla}_{b} \hat{\nabla}^{[d} T^{-e f] c}
\end{aligned}
$$




\subsection{The supersymmetric $F \square F$ invariant in components}

The supersymmetrization of the $F \square F$ action follows a similar path as $C \square C$, although in this case the calculations involved are significantly simpler. We will need the description of an off-shell Yang-Mills multiplet coupled to conformal supergravity. This was given in conformal superspace in appendix $\mathrm{C}$ of [9] where we refer the reader for more details.

Let us first briefly review and elaborate on the basic ingredients that we need for our analysis. The non-abelian vector multiplet is described by a dimension $3 / 2$ conformal primary spinor field strength superfield, $\Lambda^{\alpha i I}$, satisfying the constraints $[9,26,54]$ :

$$
S_{i}^{\alpha} \Lambda^{\gamma k I}=0, \quad \mathbb{D} \Lambda^{\gamma k I}=\frac{3}{2} \Lambda^{\gamma k I}, \quad \nabla_{\gamma}^{k} \Lambda_{k}^{\gamma I}=0, \quad \nabla_{\alpha}^{(i} \Lambda^{\beta j) I}=\frac{1}{4} \delta_{\alpha}^{\beta} \nabla_{\gamma}^{(i} \Lambda^{\gamma j) I} .
$$

The index $I$ is in the adjoint, and associated with $\Lambda^{\alpha i I}$ is a matrix valued in the Lie algebra of the gauge group via $\boldsymbol{\Lambda}^{\alpha i}=\Lambda^{\alpha i I} \mathbf{t}_{I}$ with $\mathbf{t}_{I}$ the Hermitian generators of the unitary gauge group, obeying $\left[\mathbf{t}_{I}, \mathbf{t}_{J}\right]=-\mathrm{i} f_{I J}{ }^{K} \mathbf{t}_{K}$. (The generalization to non-unitary gauge groups is obvious.) Here the covariant derivatives $\hat{\nabla}_{A}=\left(\hat{\nabla}_{a}, \nabla_{\alpha}^{i}\right)=E_{A}-\hat{\omega}_{A} \underline{\underline{b}} X_{b}-\mathrm{i} \boldsymbol{V}_{A}$ carry the additional Yang-Mills connections $\boldsymbol{V}:=E^{A} \boldsymbol{V}_{A}=E^{A} V_{A}{ }^{I} \mathbf{t}_{I}$. Their algebra is

$$
\left[\hat{\nabla}_{A}, \hat{\nabla}_{B}\right\}=-\hat{T}_{A B}^{C} \hat{\nabla}_{C}-\hat{R}_{A B}{ }^{\underline{c}} X_{\underline{c}}-\mathrm{i} \mathcal{F}_{A B},
$$

where the torsion and curvatures are those of conformal superspace and $\mathcal{F}_{A B}{ }^{I}$ is the field strength two-form. In terms of the primary superfield $\Lambda^{\alpha i I}$ the components of $\mathcal{F}_{A B}{ }^{I}$ are

$$
\mathcal{F}_{\alpha \beta}^{i j I}=0, \quad \mathcal{F}_{a_{\beta}}^{j}=\left(\gamma_{a}\right)_{\alpha \beta} \Lambda^{\beta i I}, \quad \mathcal{F}_{a b}{ }^{I}=-\frac{\mathrm{i}}{8}\left(\gamma_{a b}\right)_{\beta}{ }^{\alpha} \nabla_{\alpha}^{k} \Lambda_{k}^{\beta I} .
$$

The various component fields are defined as follows. The gaugino of the vector multiplet is given by the projection $\Lambda^{\alpha i I} \mid$. The component one-form $v_{m}{ }^{I}$ and its field strength $F_{m n}{ }^{I}$ are given by $V_{m}{ }^{I} \mid$ and $\mathcal{F}_{m n}{ }^{I}$, respectively. The supercovariant field strength $\mathcal{F}_{a b}{ }^{I}$ is given simply by $\mathcal{F}_{a b}{ }^{I}$, and as usual one finds that $\mathcal{F}_{a b}{ }^{I}$ and $F_{m n}{ }^{I}$ differ by gravitino terms,

$$
\mathcal{F}_{a b}^{I}=e_{a}^{m} e_{b}^{n} F_{m n}^{I}+\psi_{[a k} \gamma_{b]} \Lambda^{k I}
$$

The last physical field of the vector multiplet is a Lorentz scalar and SU(2) triplet associated with the bar-projection of the following descendant superfield

$$
X^{i j I}:=\frac{\mathrm{i}}{4} \nabla_{\gamma}^{(i} \Lambda^{\gamma j) I} .
$$

In this subsection, we will denote the covariant components with exactly the same name as the associated superfield and avoid the explicit bar-projection.

The superfields $\Lambda^{\alpha i I}, X^{i j I}$, together with $\mathcal{F}_{\alpha}{ }^{\beta I}=-\frac{1}{4}\left(\gamma^{a b}\right)_{\alpha}{ }^{\beta} \mathcal{F}_{a b}{ }^{I}$, are all annihilated by $K_{a}$ and satisfy the following useful identities:

$$
\begin{aligned}
& \nabla_{\alpha}^{i} \Lambda^{\beta j I}=-\mathrm{i} \delta_{\alpha}^{\beta} X^{i j I}-2 \mathrm{i} \varepsilon^{i j} \mathcal{F}_{\alpha}^{\beta I}, \quad \nabla_{\alpha}^{i} X^{j k I}=2 \varepsilon^{i(j} \hat{\nabla}_{\alpha \beta} \Lambda^{\beta k) I}, \\
& \nabla_{\alpha}^{i} \mathcal{F}_{\beta}^{\gamma I}=-\hat{\nabla}_{\alpha \beta} \Lambda^{\gamma i I}-\delta_{\alpha}^{\gamma} \hat{\nabla}_{\beta \delta} \Lambda^{\delta i I}+\frac{1}{2} \delta_{\beta}^{\gamma} \hat{\nabla}_{\alpha \delta} \Lambda^{\delta i I}-\varepsilon_{\alpha \beta \rho \tau} W^{\gamma \rho} \Lambda^{\tau i I} \\
& S_{k}^{\gamma} \mathcal{F}_{\alpha}^{\beta I}=-4 \mathrm{i} \delta_{\alpha}^{\gamma} \Lambda_{k}^{\beta I}+\mathrm{i} \delta_{\alpha}^{\beta} \Lambda_{k}^{\gamma I}, \quad S_{k}^{\gamma} X^{i j I}=-4 \mathrm{i} \delta_{k}^{(i} \Lambda^{\gamma j) I} .
\end{aligned}
$$


These imply that the $\delta=\delta_{Q}+\delta_{S}+\delta_{K}$ transformations of the component fields are

$$
\begin{aligned}
\delta \Lambda^{j I} & =-\mathrm{i} \xi_{i} X^{i j I}+\frac{\mathrm{i}}{2} \tilde{\gamma}^{a b} \xi^{j} \mathcal{F}_{a b}{ }^{I}, \\
\delta X^{j k I} & =-2 \xi^{(j} \hat{\nabla} \Lambda^{k) I}-4 \mathrm{i} \eta^{(j} \Lambda^{k) I}, \\
\delta \mathcal{F}_{a b}{ }^{I} & =2 \xi_{i} \gamma_{[a} \hat{\nabla}_{b]} \Lambda^{i I}+T_{a b c}^{-} \xi_{i} \gamma^{c} \Lambda^{i I}+2 \mathrm{i} \eta^{i} \tilde{\gamma}_{a b} \Lambda_{i}{ }^{I},
\end{aligned}
$$

where

$$
\begin{aligned}
\hat{\nabla}_{a} \Lambda^{i I} & =\hat{\mathcal{D}}_{a} \Lambda^{i I}+\frac{\mathrm{i}}{2} X^{i j I} \psi_{a j}-\frac{\mathrm{i}}{4} \tilde{\gamma}^{b c} \psi_{a}{ }^{i} \mathcal{F}_{b c}{ }^{I} \\
\hat{\mathcal{D}}_{a} & :=e_{a}{ }^{m} \partial_{m}-\frac{1}{2} \hat{\omega}_{a}{ }^{c d} M_{c d}-b_{a} \mathbb{D}-\mathcal{V}_{a}{ }^{k l} J_{k l}-\mathrm{i} \boldsymbol{v}_{a}
\end{aligned}
$$

The transformation rule of the component connection $v_{m}{ }^{I}$ can be derived from the supergravity gauge transformation of $V^{I}, \delta_{\mathcal{G}} V^{I}=E^{B} \xi^{C} \mathcal{F}_{C B}{ }^{I}$, leading to

$$
\delta v_{m}^{I}=-\xi_{k} \gamma_{m} \Lambda^{k I}
$$

Up to differences in conventions, these match the results of [30].

To construct the $F \square F$ action, we again exploit the $B$-action principle. Here the relevant composite superfield is

$$
B_{a}{ }^{i j}=\frac{\mathrm{i}}{4} \operatorname{Tr}\left(\boldsymbol{\Lambda}^{i} \gamma_{a} \Lambda^{j}\right)=\frac{\mathrm{i}}{4} \Lambda^{i I} \gamma_{a} \Lambda^{j J} g_{I J},
$$

where $g_{I J}$ is the Cartan-Killing metric, which we employ to raise and lower adjoint indices. This superfield again describes a composite four-form field strength multiplet, with the bosonic part of $C_{[a b]}$ given by

$$
C_{[a b]}=-\frac{1}{8} \varepsilon_{a b c d e f} F^{c d I} F^{e f}{ }_{I},
$$

which is indeed the dual of a closed four-form. The full expressions for the various pieces of the $B$ action principle are given in a supplementary file.

Building the bosonic Lagrangian as in (4.9) and dropping a total derivative leads to

$$
\begin{aligned}
\mathcal{L}= & -F_{a b}{ }^{I} \hat{\nabla}^{2} F^{a b}{ }_{I}+X^{i j K} \hat{\nabla}^{2} X_{i j K}-\frac{2}{15} D F_{a b}{ }^{I} F^{a b}{ }_{I}+\frac{2}{5} D X^{i j K} X_{i j K} \\
& +4 F_{a b}{ }^{I} F^{c d}{ }_{I} T^{-a b e} T_{c d e}^{-}+C^{a b c d} F_{a b}{ }^{I} F_{c d I}+8 T^{-a b c} F_{a b}{ }^{I} \hat{\nabla}^{d} F_{c d I}+4 T^{-a c d} F_{a b}{ }^{I} \hat{\nabla}^{b} F_{c d I} \\
& +2 F^{a b I}{ }^{I} X^{i j}{ }_{I} \hat{R}(J)_{a b i j}-2 F_{a}{ }^{b I} F_{b}{ }^{c J} F_{c}{ }^{a K} f_{I J K}+X_{i}{ }^{I I} X_{j}{ }^{k J} X_{k}{ }^{i K} f_{I J K} \\
& +4 \hat{\mathfrak{f}}_{a}{ }^{a} F_{b c}{ }^{I} F^{b c}{ }_{I}-8 \hat{\mathfrak{f}}_{a}{ }^{b} F_{b c}{ }^{I} F^{a c}{ }_{I} .
\end{aligned}
$$

Some useful results, which hold up to fermions, are

$$
\begin{aligned}
& \hat{\nabla}_{a} \hat{\nabla}_{b} X^{i j I}=\hat{\mathcal{D}}_{a} \hat{\mathcal{D}}_{b} X^{i j I}-4 \hat{f}_{a b} X^{i j I} \\
& \hat{\nabla}_{a} \hat{\nabla}_{b} F_{c d}{ }^{I}=\hat{\mathcal{D}}_{a} \hat{\mathcal{D}}_{b} F_{c d}{ }^{I}-4 \hat{f}_{a b} F_{c d}^{I}+4 \hat{f}_{a[c} F_{d] b}{ }^{I}-4 \hat{f}_{a}^{e} F_{e[c}{ }^{I} \eta_{d] b} .
\end{aligned}
$$




\section{The $\mathcal{N}=(2,0)$ conformal supergravity invariant}

As mentioned in the introduction, it is expected that there should be only one type B anomaly for $\mathcal{N}=(2,0)$ superconformal field theories with a specific relative coefficient between the bosonic $C \square C$ and curvature cubed terms. The type B anomaly should correspond to some $\mathcal{N}=(2,0)$ conformal supergravity invariant. In this section, assuming such an invariant exists, we provide an alternative argument for its uniqueness (and, therefore, of the type B anomaly) and significantly extend its known bosonic terms.

The Weyl multiplet for $\mathcal{N}=(2,0)$ conformal supergravity was constructed in [55]. There it was noted that many of the formulae involving the $\mathcal{N}=(2,0)$ Weyl multiplet can actually be obtained by considering their truncations to the $\mathcal{N}=(1,0)$ case. It turns out that most of the bosonic part of the $\mathcal{N}=(2,0)$ conformal supergravity invariant can be reverse-engineered in a similar way by considering its potential $\mathcal{N}=(1,0)$ reduction. A key issue is that while there exist two $(1,0)$ conformal supergravity invariants, only one $(2,0)$ invariant is expected. It is immediately apparent that neither of the two $(1,0)$ invariants described in the previous sections can alone originate from the truncation of a $(2,0)$ invariant. The reason for this derives from the presence of e.g. the term $D C^{a b c d} C_{a b c d}$ appearing in these actions, which cannot arise from the truncation of a scalar term in the $\mathcal{N}=(2,0)$ case; the covariant scalar field in the $(2,0)$ Weyl multiplet is $D^{i j} k l$, which lies in the $\mathbf{1 4}$ of the USp(4) $R$-symmetry group, and so no such term can be built as a USp(4) singlet. However, there exists a certain linear combination of the two $(1,0)$ invariants for which all such terms cancel and it is this combination which could come from a potential truncation. It is worth emphasizing that since any potential $(2,0)$ conformal supergravity invariant has a $(1,0)$ truncation, this leads to a proof that there can be at most one $(2,0)$ conformal supergravity invariant. In order to see this in more detail and uncover many of the bosonic terms in the $(2,0)$ conformal supergravity invariant it is necessary to first briefly review the salient details of the $(1,0)$ truncation of the $(2,0)$ Weyl multiplet (slightly adapted to our notation and conventions).

To begin with let us recall the component structure of the Weyl multiplet of $\mathcal{N}=(2,0)$ conformal supergravity [55]. The superconformal tensor calculus of [55] is based on an off-shell gauging of the $\mathcal{N}=(2,0)$ superconformal group. One associates the following independent fields with the local translations, $Q$-supersymmetry, $\operatorname{USp}(4) R$-symmetry, and the dilatations: the vielbein $e_{m}{ }^{a}$, the gravitino $\psi_{m}{ }^{i}$, the $\operatorname{USp}(4)$ gauge field $\mathcal{V}_{m}{ }^{i j}$, and the dilatation gauge field $b_{m}$. The remaining gauge symmetries are associated with composite connections, which include the spin connection $\omega_{m}{ }^{c d}$, the $S$-supersymmetry connection $\phi_{m}{ }^{i}$ and the special conformal connection $\mathfrak{f}_{m a}$. An off-shell representation of the conformal supersymmetry algebra is achieved by introducing three covariant matter fields: $T_{a b c}{ }^{i j}=$ $T_{[a b c]}^{[i j]}, \chi^{i, j k}=\chi^{i,[j k]}$ and $D^{i j}{ }_{k l}=D^{[i j]}{ }_{[k l]}=D_{k l}{ }^{i j}$. Here $T_{a b c}{ }^{i j}$ is anti-self-dual with respect to its Lorentz vector indices and all covariant matter fields of the Weyl multiplet are traceless with respect to the invariant antisymmetric tensor $\Omega^{i j}$ of $\operatorname{USp}(4)$. These covariant fields are used to build the full covariant curvatures given in [55]. In this section we have endeavored to match the conventions of [55], but the reader should keep in mind some minor differences explained later. Here we do not provide details such as the supersymmetry transformations and expressions for the composite fields since these are given in [55]. 
We now wish to outline how to perform the truncation. The $(2,0)$ Weyl multiplet should decompose into the following $(1,0)$ multiplets: a Weyl multiplet on which half of the supersymmetry is manifest, two gravitini multiplets associated with the extra spin-3/2 gauge fields, and a Yang-Mills multiplet associated with the extra $R$-symmetry connections. The structure of the additional gravitino multiplets and their couplings will be quite complicated from a $(1,0)$ perspective, so we will switch them off. However, we will elect to keep the additional $(1,0)$ Yang-Mills multiplet, which takes its values in an $\mathrm{SU}(2)$ gauge group, rather than turning it off (as in the analysis of [55]). This means that the truncation of the $\mathcal{N}=(2,0)$ conformal supergravity action should also generate the $F \square F$ invariant in a linear combination with the $\mathcal{N}=(1,0)$ conformal supergravity invariants. This will in turn provide a useful consistency check on our results.

The truncation follows [55] very closely. We split the USp(4) indices $i=1, \cdots, 4$ to $\left(i=1,2, i^{\prime}=1,2\right)$ and switch off the third and fourth gravitini $\psi_{m}{ }^{i^{\prime}}=0$. To preserve this last condition, we must restrict $\mathrm{USp}(4)$ transformations to the block diagonal form

$$
\Lambda_{j}^{i}=\left(\begin{array}{cc}
\Lambda_{j}^{i} & 0 \\
0 & \Lambda^{i^{\prime}}{ }_{j^{\prime}}
\end{array}\right)
$$

where we have chosen a basis for $\Omega^{i j}$ so that ${ }^{12}$

$$
\Omega^{i j}=\left(\begin{array}{cc}
\varepsilon^{i j} & 0 \\
0 & \varepsilon^{i^{\prime} j^{\prime}}
\end{array}\right), \quad \Omega_{i j}=\left(\begin{array}{cc}
\varepsilon_{i j} & 0 \\
0 & \varepsilon_{i^{\prime} j^{\prime}}
\end{array}\right) .
$$

The above conditions ensure that $\Lambda^{i}{ }_{j}$ and $\Lambda^{i^{\prime}}{ }_{j^{\prime}}$ parametrize $\mathrm{SU}(2) \times \mathrm{SU}(2)$ local gauge transformations. Considering the supersymmetry transformation of the gravitini one must also impose $V_{m}^{i j^{\prime}}=0$. We keep separately the $\mathrm{SU}(2)$ gauge fields $V_{m}{ }^{i j}=\hat{\mathcal{V}}_{m}{ }^{i j}$ and $V_{m}{ }^{i^{\prime} j^{\prime}}$ in what follows. Since we have turned off the extra gravitini, it is necessary to constrain some of the covariant fields so that the $Q$ - and $S$-supersymmetry transformations are consistent. ${ }^{13}$ The non-vanishing covariant fields are

$$
\begin{aligned}
T_{a b c}{ }^{i j} & =\varepsilon^{i j} T_{a b c}^{-}, \quad T_{a b c}{ }^{i^{\prime} j^{\prime}}=-\varepsilon^{i^{\prime} j^{\prime}} T_{a b c}^{-}, \\
\chi_{i}{ }^{j k} & =\varepsilon^{j k} \chi_{i}, \quad \chi_{i}^{j^{\prime} k^{\prime}}=-\varepsilon^{j^{\prime} k^{\prime}} \chi_{i}, \quad \chi_{i^{\prime}}{ }^{j^{\prime} k}=-\frac{15}{4}\left(\Omega^{k}\right)_{i^{\prime}}^{j^{\prime}}+\frac{1}{2} \delta_{i^{\prime}}^{j^{\prime}} \chi^{k}, \\
D_{k l}^{i j} & =-\varepsilon^{i j} \varepsilon_{k l} D, \quad D^{i j}{ }_{k^{\prime} l^{\prime}}=\varepsilon^{i j} \varepsilon_{k^{\prime} l^{\prime}} D, \quad D^{i^{\prime} j^{\prime}}{ }_{k^{\prime} l^{\prime}}=-\varepsilon^{i^{\prime} j^{\prime}} \varepsilon_{k^{\prime} l^{\prime}} D, \\
D_{k l^{\prime}}^{i j^{\prime}} & =-\frac{1}{2} \delta_{k}^{i} \delta_{l^{\prime}}^{j^{\prime}} D+\frac{15}{2}\left(Y_{k}^{i}\right)^{j^{\prime}}{ }_{l^{\prime}},
\end{aligned}
$$

where $\left(\Omega^{k}\right)^{i^{\prime} j^{\prime}},\left(Y^{i j}\right)^{i^{\prime} j^{\prime}}$ are the covariant component fields of the additional SU(2) YangMills multiplet.

It is important to keep in mind that the conventional constraints chosen for the $(2,0)$ Weyl multiplet in [55] are not "traceless", because there is a contribution of the form

\footnotetext{
${ }^{12}$ Our conventions for $\varepsilon_{i j}$ and $\Omega_{i j}$ differ by a sign from the ones of [55]. However, our conventions for lowering a USp(4) index also differ by a sign so lowering a $\operatorname{USp}(4)$ index as $\lambda_{i}=\Omega_{i j} \lambda^{j}$ is actually equivalent to lowering the index in the conventions of [55].

${ }^{13}$ We take as in [55] the truncated supersymmetry parameters $\epsilon^{i} \rightarrow\left(\epsilon^{i}, 0\right)$ and $\eta^{i} \rightarrow\left(\eta^{i}, 0\right)$.
} 
$T_{a c d}{ }^{i j} T^{b c d}{ }_{i j}$ to the $(2,0)$ special conformal gauge field $\mathfrak{f}_{a}{ }^{b}$. Thus upon truncating, one would have to switch to the "traceless" frame by extracting the term quadratic in $T_{a b c}^{-}$in order to match formulae. We will deal with this by writing all $(2,0)$ formulae below in terms of a "traceless" $(2,0)$ gauge field $\hat{\mathfrak{f}}_{a}^{b}:=\mathfrak{f}_{a}{ }^{b}-\frac{1}{32} T_{a c d i j} T^{b c d i j}$.

Now by considering the truncation of various terms built out of the $\mathcal{N}=(2,0)$ Weyl multiplet, one can identify the linear combination of the supersymmetric $C^{3}, C \square C$ and $F \square F$ invariants that permits an uplift to $(2,0)$. This combination is

$$
I_{C \square C}+\frac{1}{2} I_{C^{3}}+I_{F \square F},
$$

where the additional $\mathrm{SU}(2)$ generators are taken in the fundamental so that i $V_{m}{ }^{I}\left(t_{I}\right)^{i^{\prime}{ }_{j^{\prime}}=}$ $V_{m}{ }^{i^{\prime}}{ }^{\prime}$. As already described, this linear combination of $C \square C$ and $C^{3}$ is the only choice that eliminates the $D C^{a b c d} C_{a b c d}$ term appearing in both the $(1,0)$ invariants. The additional contribution $I_{F \square F}$ can be determined by uplifting the first two invariants to $(2,0)$ and then reducing back to $(1,0)$; this actually provides an independent check of the entire $I_{F \square F}$ invariant. The result of the uplift gives most of the bosonic terms in the corresponding $\mathcal{N}=(2,0)$ conformal supergravity invariant:

$$
\begin{aligned}
& \mathcal{L}=\frac{1}{3} C_{a b c d} \hat{\nabla}^{2} C^{a b c d}+C_{a b}{ }^{c d} C^{a b e f} C_{c d e f}-4 C_{a b c d} C^{a e c f} C^{b} e^{d}{ }_{f} \\
& \text { - R(V })_{a b}{ }^{i j} \hat{\nabla}^{2} R(V)^{a b}{ }_{i j}-2 R(V)_{a}{ }^{b}{ }_{i}{ }^{j} R(V)^{a}{ }_{c j}{ }^{k} R(V)_{b}{ }^{c}{ }_{k}{ }^{i}+C^{a b c d} R(V)_{a b}{ }^{i j} R(V)_{c d i j} \\
& +\hat{\mathfrak{f}}_{a}{ }^{b}\left(\frac{32}{3} C^{a c d e} C_{b c d e}-8 R(V)_{b c}{ }^{i j} R(V)^{a c}{ }_{i j}\right)-4 \hat{\mathfrak{f}}_{a}{ }^{a}\left(C_{b c d e} C^{b c d e}-R(V)_{b c}{ }^{i j} R(V)^{b c}{ }_{i j}\right) \\
& +\frac{1}{225} D^{i j}{ }_{k l} \hat{\nabla}^{2} D^{k l}{ }_{i j}-\frac{2}{3375} D^{i j}{ }_{k l} D^{k l}{ }_{p q} D^{p q}{ }_{i j}-\frac{2}{15} D^{i j}{ }_{k l} R(V)_{a b}{ }_{i} R(V)^{a b j}{ }_{l} \\
& +4 T_{a b c}{ }^{i j} \hat{\nabla}_{d} R(V)^{a b}{ }_{j k} R(V)^{c d k}{ }_{i}+8 T_{a b c}{ }^{i j} \hat{\nabla}_{d} R(V)^{a d}{ }_{j k} R(V)^{b c k}{ }_{i} \\
& -T_{a b c}{ }^{i j} \Delta^{4} T^{a b c}{ }_{i j}+\frac{8}{3} C_{a b c d} T^{a b e}{ }_{i j} \hat{\nabla}_{e} \hat{\nabla}_{f} T^{c d f i j}+\frac{4}{3} C_{a b c d} T^{a b e}{ }_{i j} \hat{\nabla}_{f} \hat{\nabla}_{e} T^{c d f i j} \\
& -\frac{8}{3} C^{a b c d} T_{a e f i j} \hat{\nabla}_{b} \hat{\nabla}_{c} T_{d}{ }^{e f i j}+2 C_{a b}{ }^{c d} \hat{\nabla}^{a} T^{b e f}{ }_{i j} \hat{\nabla}_{c} T_{d e f}{ }^{i j}+3 C_{a b}{ }^{c d} \hat{\nabla}_{e} T^{a b f}{ }_{i j} \hat{\nabla}_{f} T^{c d e i j} \\
& -\frac{4}{3} C_{a b e f} C^{c d e f} T_{a b g}{ }^{i j} T^{c d g}{ }_{i j}+4 \alpha T_{a b c}{ }^{i j} T^{a d e k l} R(V)^{b c}{ }_{i k} R(V)_{d e j l} \\
& +2(1-\alpha) T_{a b c}{ }^{i j} T^{a d e}{ }_{i j} R(V)^{b c}{ }_{k l} R(V){ }_{d e}{ }^{k l} \\
& +\frac{2}{15} D_{k l}^{i j}\left(T_{a b c}{ }^{k l} \hat{\nabla}^{a} \hat{\nabla}_{d} T^{b c d}{ }_{i j}-\frac{1}{2} \hat{\nabla}^{a} T_{a b c}{ }^{k l} \hat{\nabla}_{d} T^{b c d}{ }_{i j}\right) \\
& -\frac{1}{60} D^{i j}{ }_{k l} T_{a b c}{ }^{k l} T^{a b d}{ }_{i j} T^{c e f}{ }_{p q} T_{d e f}{ }^{p q}+\mathcal{O}\left(T^{4}\right),
\end{aligned}
$$

where we have introduced $\hat{\nabla}_{a}:=\mathcal{D}_{a}-\hat{\mathfrak{f}}_{a}^{b} K_{b}$, and the $K$-invariant operator $\Delta^{4}$ is defined formally the same as (4.16). This action is exactly determined up to linear order in the covariant field $T_{a b c}{ }^{i j}$, while one combination of two terms quadratic in $T_{a b c}{ }^{i j}$ is left undetermined by the uplift and parametrized by an unknown real constant $\alpha$. No terms cubic in $T_{a b c}{ }^{i j}$ may appear for group-theoretic reasons, and indeed one finds that in the corresponding $(1,0)$ invariant $(5.4)$, all terms cubic in $T_{a b c}^{-}$cancel. There is only one possible term involving four $T_{a b c}{ }^{i j}$ and a single $D^{i j}{ }_{k l}$, which we have given here explicitly since it can 
be determined by its $(1,0)$ descendant. All other terms involving four $T_{a b c}{ }^{i j}$ are denoted here $\mathcal{O}\left(T^{4}\right)$ : these involve terms with four $T_{a b c}{ }^{i j}$ and two derivatives (or a Weyl tensor or a USp(4) curvature tensor) and cannot be uniquely determined by matching to $(1,0)$.

Note that our $(2,0)$ conventions differ from [55] in the same way as our $(1,0)$ conventions differ from [30], see appendix B. In particular, one should keep in mind a sign difference in the Lorentz curvature tensor from the one in [55], as well as the redefinition of the special conformal gauge field. Finally, the USp(4) gauge field and curvature are scaled by a factor of 2 so that

$$
R(V)_{a b}^{k l}=2 e_{a}{ }^{m} e_{b}{ }^{n}\left(\partial_{[m} V_{n]}^{k l}+V_{[m}{ }^{j(k} V_{n]}^{l)}{ }_{j}\right) .
$$

\section{Discussion}

In this paper we have described the component actions for the two conformal supergravity invariants constructed in [9]. As shown by a supercurrent analysis [9], all conformal supergravity invariants must be given by the linear combination $I=a I_{C^{3}}+b I_{C \square C}$, where $a$ and $b$ are some constants, and $I_{C^{3}}$ and $I_{C \square C}$ are the supersymmetric $C^{3}$ and $C \square C$ invariants. The relative coefficients of the three purely gravitational pieces (1.1) then obey the linear relation already known in the literature, see e.g. [3] and references therein. As discussed in the previous section, amongst the invariants there exists a special choice which corresponds to the $\mathcal{N}=(1,0)$ truncation of the $\mathcal{N}=(2,0)$ conformal supergravity invariant. Identifying the choice of coefficients as the one-parameter family that permits an uplift to $\mathcal{N}=(2,0)$ supergravity allowed us to prove uniqueness of the $(2,0)$ conformal supergravity invariant and construct many of its bosonic terms. It would be interesting to develop an alternative method to construct this invariant and recover all terms. This would be interesting not only in the context of Weyl anomalies, but also in the context of higher-derivative gravity theories [56-58], where the combination (1.4) of Weyl invariants particular to $(2,0)$ theories was observed to correspond to $6 \mathrm{D}$ critical gravity. It would be interesting to understand how the covariant matter fields of the standard Weyl multiplet affect the dynamics of these models when extended to supergravity. We expect that the development of $(2,0)$ conformal superspace in six dimensions together with the ideas advocated in [9] for the $(1,0)$ case will provide a viable means to complete the construction of the $(2,0)$ invariant. We hope the results in this paper will be useful for these applications.

While the supersymmetric $C^{3}$ and $C \square C$ invariants have been constructed in the standard Weyl multiplet, it is interesting to note that one could construct these invariants with a variant Weyl multiplet, known as the dilaton-Weyl multiplet (or type II formulation) [30]. ${ }^{14}$ The dilaton-Weyl multiplet is obtained by coupling the standard Weyl multiplet to a tensor multiplet $[59,60]$ and exchanging the covariant matter fields of the standard Weyl multiplet with those of the tensor multiplet, which include a scalar field $\sigma$, a gauge 2 -form $B_{a b}$, and a negative chirality spinor $\chi_{\alpha}^{i}$. This procedure can directly be performed for the supersymmetric $C^{3}$ and $C \square C$ invariants and would lead to new higher-derivative invariants in the dilaton-Weyl multiplet.

\footnotetext{
${ }^{14}$ See e.g. [37, 38] for a recent discussion.
} 
Because the dilaton-Weyl multiplet possesses a built-in Weyl compensator in the form of the scalar field $\sigma$, it is evident that there are many other curvature invariants one can construct that have no analogues in the standard Weyl multiplet. For example, one can construct a supersymmetric $\mathcal{R}_{a b c d} \square \mathcal{R}^{a b c d}$ action in addition to $C_{a b c d} \square C^{a b c d}$. To see this, it is important to realize that the supersymmetry transformations of certain fields built out of the dilaton-Weyl multiplet can be formally mapped to those of a Yang-Mills multiplet taking values in the 6D Lorentz algebra [36]. This property leads to a natural correspondence between an action based on a Yang-Mills multiplet and an action in terms of the dilaton-Weyl multiplet. Using this correspondence (given in component form in [36]), the supersymmetric $F \square F$ action can be converted into an invariant containing $\mathcal{R}_{a b c d} \square \mathcal{R}^{a b c d}$.

It is worth mentioning that the YM correspondence can also be exhibited in superspace. First, one must adapt superspace to the dilaton-Weyl multiplet by introducing a compensating tensor multiplet $\Phi$ (with lowest component $\sigma$ ), which satisfies $\nabla_{\alpha}^{(i} \nabla_{\beta}^{j)} \Phi=0$, and then make use of the modified derivatives $\mathscr{D}_{\alpha}^{i}$ and the associated torsion components that were introduced in section 3.4 of [9] (with $X=\Phi) .{ }^{15}$ One can then construct a primary superfield $\boldsymbol{\Lambda}^{\alpha i}{ }_{\beta}^{\gamma}$ satisfying the following constraints (which are formally the same constraints as those of a vector multiplet valued in the Lorentz group): ${ }^{16}$

$$
\boldsymbol{\Lambda}_{\beta}^{\alpha i}{ }_{\beta}^{\beta}=0, \quad \mathscr{D}_{\alpha}^{(i} \boldsymbol{\Lambda}^{\beta j)} \gamma^{\delta}-\frac{1}{4} \delta_{\alpha}^{\beta} \mathscr{D}_{\rho}^{(i} \boldsymbol{\Lambda}_{\gamma}^{\rho j)} \gamma^{\delta}=0, \quad \mathscr{D}_{\alpha i} \boldsymbol{\Lambda}_{\beta}^{\alpha i}{ }_{\beta}^{\gamma}=0 .
$$

The appropriate primary superfield $\boldsymbol{\Lambda}_{\beta}^{\alpha i}{ }^{\gamma}$ is given by

$$
\Lambda^{\alpha i}:=\Lambda_{\beta}^{\alpha i}{ }_{\beta}^{\gamma}{ }_{\gamma}^{\beta}=\Phi^{3 / 4}\left(\mathscr{D}_{\beta}^{i} \mathscr{W}^{\alpha \gamma}-\frac{2}{3} \varepsilon^{\alpha \gamma \delta \rho} \mathscr{D}_{\delta}^{i} \mathscr{N}_{\rho \beta}-\frac{1}{3} \delta_{\beta}^{\alpha} \mathscr{D}_{\delta}^{i} \mathscr{W}^{\gamma \delta}\right) M_{\gamma}^{\beta}
$$

We can now describe a supersymmetric $\mathcal{R}_{a b c d} \square \mathcal{R}^{a b c d}$ invariant in the dilaton-Weyl multiplet in a completely analogous way as the supersymmetric $F \square F$ action by using the $B$ action principle with $B^{\alpha \beta i j}=\mathrm{i} \operatorname{Tr}\left(\boldsymbol{\Lambda}^{\alpha(i} \boldsymbol{\Lambda}^{\beta j)}\right)$. It would be interesting to carry out a detailed analysis of this supersymmetric invariant elsewhere. As a side note, the primary superfield (6.2) may be used to construct other invariants. For instance, one can describe a topological invariant containing the $6 \mathrm{D}$ Euler term using the $A$ action principle with $A_{\alpha}^{i j k}=\varepsilon_{\alpha \beta \gamma \delta} \operatorname{Tr}\left(\boldsymbol{\Lambda}^{\beta(i} \Lambda^{\gamma j} \boldsymbol{\Lambda}^{\delta k)}\right)$.

A natural question to ask is whether other curvature invariants with fewer than six derivatives may be built when compensating superfields, such as the tensor superfield of the dilaton-Weyl multiplet, are present. It turns out that it is possible to construct all of the curvature-squared invariants using either tensor or linear multiplet compensators, just as in five dimensions [61]. In six dimensions, there is a topological action, corresponding to the supersymmetrization of $B_{2} \wedge H_{4}$, that couples a tensor multiplet (with two-form potential $B_{2}$ ) to a four-form field strength multiplet (containing a closed four-form $H_{4}$ ). It

\footnotetext{
${ }^{15}$ The resulting superspace geometry is equivalent to using the $\mathrm{SU}(2)$ superspace formulation of conformal supergravity in [26] with the torsion component $C_{a}{ }^{i j}$ switched off. This is also equivalent to the superspace of [36].

${ }^{16}$ The superspace results given here are similar to the $5 \mathrm{D} \mathcal{N}=1$ description of the Riemann curvature squared invariant in the dilaton-Weyl multiplet given in [13].
} 
can be built by starting with the $A$ action principle with the specific choice ${ }^{17}$

$$
A_{\alpha}{ }^{i j k}=\varepsilon_{\alpha \beta \gamma \delta} V^{\beta(i} B^{\gamma \delta j k)},
$$

where $V^{\alpha i}$ is the prepotential for the tensor multiplet (see [26, 62] for details) with the superfield $B^{\alpha \beta i j}$ satisfying the constraints (4.1) and (4.10) corresponding to a four-form field strength multiplet [44]. A special form of this action was already constructed in [30], for the case where $H_{4}=\operatorname{Tr}(\boldsymbol{F} \wedge \boldsymbol{F})$ is built out of a non-abelian vector multiplet. Its component action contains the terms $\sigma \operatorname{Tr}\left(\boldsymbol{F}_{a b} \boldsymbol{F}^{a b}\right)$ and $\sigma \operatorname{Tr}\left(\boldsymbol{X}_{i j} \boldsymbol{X}^{i j}\right)$. Employing the YM correspondence, the first of these terms gives a curvature-squared invariant in the gauge $\sigma=1$ via $\operatorname{Tr}\left(\boldsymbol{F}_{a b} \boldsymbol{F}^{a b}\right) \rightarrow \mathcal{R}_{a b c d} \mathcal{R}^{a b c d}$, as shown in [36] (see also [34, 35]). This construction corresponds to choosing the superfield $B^{\alpha \beta i j}=\mathrm{i} \operatorname{Tr}\left(\boldsymbol{\Lambda}^{\alpha(i} \boldsymbol{\Lambda}^{\beta j)}\right)$ within (6.3) and imposing the gauge $\Phi=1$.

A second curvature squared invariant can be built by choosing a composite abelian vector multiplet built out of a linear multiplet $G^{i j}$ [63]. In superspace, this corresponds to taking $B^{\alpha \beta i j}=\mathrm{i} \mathbb{W}^{\alpha(i} \mathbb{W}^{\beta j)}$, with $\mathbb{W}^{\alpha i}$ built from the superfield $G^{i j}$ as

$$
\begin{aligned}
\mathbb{W}^{\alpha i}= & \frac{1}{G} \nabla^{\alpha \beta} \Upsilon_{\beta}^{i}+\frac{4}{G}\left(W^{\alpha \beta} \Upsilon_{\beta}^{i}+10 \mathrm{i} X_{j}^{\alpha} G^{j i}\right)-\frac{1}{2 G^{3}} G_{j k}\left(\nabla^{\alpha \beta} G^{i j}\right) \Upsilon_{\beta}^{k}+\frac{1}{2 G^{3}} G^{i j} F^{\alpha \beta} \Upsilon_{\beta j} \\
& +\frac{\mathrm{i}}{16 G^{5}} \varepsilon^{\alpha \beta \gamma \delta} \Upsilon_{\beta j} \Upsilon_{\gamma k} \Upsilon_{\delta l} G^{i j} G^{k l},
\end{aligned}
$$

where $\Upsilon_{\alpha}^{i}:=\frac{2}{3} \nabla_{\alpha j} G^{i j}$ and $F_{\alpha \beta}:=\frac{i}{4} \nabla_{[\alpha}^{k} \Upsilon_{\beta] k}$. Provided $G^{i j}$ satisfies the linear multiplet constraint $\nabla_{\alpha}^{(i} G^{j k)}=0$, $\mathbb{W}^{\alpha i}$ describes a composite abelian vector multiplet. ${ }^{18}$ The isotriplet $\mathbb{X}^{i j}$ turns out to include a term $\mathcal{R} G^{i j} / G$, and the term $\mathbb{X}_{i j} \mathbb{X}^{i j}$ within the component action generates $\mathcal{R}^{2}$.

A third curvature-squared invariant is possible but unlike the previous two examples, it actually requires the supersymmetric version of $B_{2} \wedge H_{4}$ where the four-form field strength is not a product of Yang-Mills curvature two-forms. One must take the four-form multiplet built from the superfield $B^{\alpha \beta i j}$ given in (4.11). It is not hard to see that the component action must contain a term $C_{a b c d} C^{a b c d}$, which then completes the set of curvature-squared invariants. Because the use of the four-form field strength multiplet has to our knowledge not been considered, the last curvature-squared invariant remained undiscovered until now. The three curvature squared invariants described here, corresponding to supersymmetric extensions of $\mathcal{R}_{a b c d} \mathcal{R}^{a b c d}, \mathcal{R}^{2}$, and $C_{a b c d} C^{a b c d}$, extend the analogous $5 \mathrm{D}$ examples constructed in components [61] and superspace [13], and span the supersymmetric extensions of all possible curvature squared terms. Another curvature-squared invariant was partially constructed in $[34,35]$ and may correspond to a linear combination of these. It would also be interesting to find a connection with the curvature-squared invariants of [65] involving supergravity coupled to matter. We leave a further discussion and analysis of these invariants, including the component expression for $C_{a b c d} C^{a b c d}$, for future work.

\footnotetext{
${ }^{17}$ We refer the reader to [9] for details about the gauge invariance of this action.

${ }^{18}$ The component form of the vector multiplet $\mathbb{W}^{\alpha i}$ appeared originally in [30]. The corresponding result in Minkowski superspace was given in [64].
} 


\section{Acknowledgments}

We thank Sergei M. Kuzenko and Stefan Theisen for discussions and collaboration at an early stage of this work. The work of DB was supported in part by the ERC Advanced Grant no. 246974, "Supersymmetry: a window to non-perturbative physics" and by the European Commission Marie Curie International Incoming Fellowship grant no. PIIF-GA2012-627976. JN acknowledges support from GIF — the German-Israeli Foundation for Scientific Research and Development. The work of GT-M is supported by the Interuniversity Attraction Poles Programme initiated by the Belgian Science Policy (P7/37), in part by COST Action MP1210 "The String Theory Universe," and was also in part supported by the Australian Research Council (ARC) Discovery Project DP140103925. DB also thanks Kasper Peeters for frequent correspondence regarding the use of Cadabra, and Ergin Sezgin and the Mitchell Institute at Texas A\&M for kind hospitality and support at the end stage of the project. GT-M is grateful to the School of Physics at the University of Western Australia for kind hospitality and support during the final part of this project. We thank the Galileo Galilei Institute for Theoretical Physics for hospitality and the INFN for partial support during the completion of this work in September 2016.

\section{A $\quad 6 \mathrm{D} \mathcal{N}=(1,0)$ conformal superspace}

In this appendix we review and expound on the $6 \mathrm{D} \mathcal{N}=(1,0)$ conformal superspace of [9] focusing on the ingredients relevant to our presentation in this paper.

\section{A.1 The superconformal algebra}

The $6 \mathrm{D} \mathcal{N}=(1,0)$ superconformal algebra contains the generators of translation $\left(P_{a}\right)$, Lorentz $\left(M_{a b}\right)$, special conformal $\left(K^{a}\right)$, dilatation $(\mathbb{D}), \mathrm{SU}(2)\left(J_{i j}\right), Q$-supersymmetry $\left(Q_{\alpha}^{i}\right)$ and $S$-supersymmetry $\left(S_{i}^{\alpha}\right)$ transformations. ${ }^{19}$ Their (anti)commutation algebra is

$$
\begin{aligned}
{\left[M_{a b}, M_{c d}\right] } & =2 \eta_{c[a} M_{b] d}-2 \eta_{d[a} M_{b] c}, \quad\left[J^{i j}, J^{k l}\right]=\varepsilon^{k(i} J^{j) l}+\varepsilon^{l(i} J^{j) k}, \\
{\left[M_{a b}, P_{c}\right] } & =2 \eta_{c[a} P_{b]}, \quad\left[M_{a b}, K_{c}\right]=2 \eta_{c[a} K_{b]}, \quad\left[\mathbb{D}, P_{a}\right]=P_{a}, \quad\left[\mathbb{D}, K_{a}\right]=-K_{a}, \\
{\left[M_{a b}, Q_{\gamma}^{k}\right] } & =-\frac{1}{2}\left(\gamma_{a b}\right)_{\gamma}{ }^{\delta} Q_{\delta}^{k}, \quad\left[\mathbb{D}, Q_{\alpha}^{i}\right]=\frac{1}{2} Q_{\alpha}^{i}, \quad\left[J^{i j}, Q_{\alpha}^{k}\right]=\varepsilon^{k(i} Q_{\alpha}^{j)}, \\
{\left[M_{a b}, S_{k}^{\gamma}\right] } & =-\frac{1}{2}\left(\tilde{\gamma}_{a b}\right)^{\gamma}{ }_{\delta} S_{k}^{\delta}, \quad\left[\mathbb{D}, S_{i}^{\alpha}\right]=-\frac{1}{2} S_{i}^{\alpha}, \quad\left[J^{i j}, S_{k}^{\alpha}\right]=\delta_{k}^{(i} S^{\alpha j)}, \\
\left\{Q_{\alpha}^{i}, Q_{\beta}^{j}\right\} & =-2 \mathrm{i} \varepsilon^{i j}\left(\gamma^{c}\right)_{\alpha \beta} P_{c}, \quad\left\{S_{i}^{\alpha}, S_{j}^{\beta}\right\}=-2 \mathrm{i} \varepsilon_{i j}\left(\tilde{\gamma}^{c}\right)^{\alpha \beta} K_{c}, \\
\left\{S_{i}^{\alpha}, Q_{\beta}^{j}\right\} & =2 \delta_{\beta}^{\alpha} \delta_{i}^{j} \mathbb{D}-4 \delta_{i}^{j} M_{\beta}{ }^{\alpha}+8 \delta_{\beta}^{\alpha} J_{i}^{j}, \quad\left[K_{a}, P_{b}\right]=2 \eta_{a b} \mathbb{D}+2 M_{a b}, \\
{\left[K_{a}, Q_{\alpha}^{i}\right] } & =-\mathrm{i}\left(\gamma_{a}\right)_{\alpha \beta} S^{\beta i}, \quad\left[S_{i}^{\alpha}, P_{a}\right]=-\mathrm{i}\left(\tilde{\gamma}_{a}\right)^{\alpha \beta} Q_{\beta i},
\end{aligned}
$$

with all other (anti)commutators vanishing. Note that the generator $M_{\alpha}{ }^{\beta}=-\frac{1}{4}\left(\gamma^{a b}\right)_{\alpha}{ }^{\beta} M_{a b}$ acts on $Q_{\gamma}^{k}$ and $S_{k}^{\gamma}$ as follows

$$
\left[M_{\alpha}^{\beta}, Q_{\gamma}^{k}\right]=-\delta_{\gamma}^{\beta} Q_{\alpha}^{k}+\frac{1}{4} \delta_{\alpha}^{\beta} Q_{\gamma}^{k}, \quad\left[M_{\alpha}^{\beta}, S_{k}^{\gamma}\right]=\delta_{\alpha}^{\gamma} S_{k}^{\beta}-\frac{1}{4} \delta_{\alpha}^{\beta} S_{k}^{\gamma} .
$$

\footnotetext{
${ }^{19}$ For our spinor conventions and notation we refer the reader to appendix A of [9].
} 
We can group together the translation and $Q$-supersymmetry as generators of supertranslations, $P_{A}=\left(Q_{\alpha}^{i}, P_{a}\right)$. Similarly we group together the special conformal and $S$-supersymmetry transformations by denoting $K^{A}=\left(S_{i}^{\alpha}, K^{a}\right)$ and the closed subset of generators that do not contain $P_{A}$ by $X_{\underline{a}}=\left(M_{a b}, J_{i j}, \mathbb{D}, K^{A}\right)$. The superconformal algebra takes the form

$$
\left[X_{\underline{a}}, X_{\underline{b}}\right\}=-f_{\underline{a} \underline{\underline{c}}}^{\underline{c}} X_{\underline{c}}, \quad\left[X_{\underline{a}}, P_{B}\right\}=-f_{\underline{a} B}{ }^{C} P_{C}-f_{\underline{a} B} \underline{c}_{\underline{c}}, \quad\left[P_{A}, P_{B}\right\}=-f_{A B}{ }^{C} P_{C}
$$

The group associated with the subalgebra generated by $X_{\underline{a}}$ is denoted by $\mathcal{H}$.

\section{A.2 Gauging the superconformal algebra in conformal superspace}

The $6 \mathrm{D} \mathcal{N}=(1,0)$ conformal superspace is parametrized by local bosonic $(x)$ and fermionic coordinates $\left(\theta_{i}\right), z^{M}=\left(x^{m}, \theta_{i}^{\mu}\right)$, where $m=0,1,2,3,4,5, \mu=1,2,3,4$ and $i=1,2$. In gauging the superconformal group we associate to each generator a connection one-form. In particular, we associate with the supertranslation $P_{A}$ a vielbein one-form $E^{A}=\left(E_{i}^{\alpha}, E^{a}\right)=$ $\mathrm{d} z^{M} E_{M}{ }^{A}$, while to the generators $X_{\underline{a}}=\left(M_{a b}, J_{i j}, \mathbb{D}, S_{i}^{\alpha}, K^{a}\right)$ we associate the connection one-forms $\omega^{\underline{a}}=\left(\Omega^{a b}, \Phi^{i j}, B, \mathfrak{F}_{\alpha}^{i}, \mathfrak{F}_{a}\right)=\mathrm{d} z^{M} \omega_{M^{\underline{a}}}=E^{A} \omega_{A^{\underline{a}}}$. These are used to construct the covariant derivatives, which have the form

$$
\nabla_{A}:=E_{A}-\omega_{A}^{\underline{c}} X_{\underline{c}}=E_{A}-\frac{1}{2} \Omega_{A}^{c d} M_{c d}-\Phi_{A}^{k l} J_{k l}-B_{A} \mathbb{D}-\mathfrak{F}_{A B} K^{B},
$$

with $E_{A}=E_{A}^{M} \partial_{M}$ the inverse vielbein.

The superconformal algebra is gauged in superspace ${ }^{20}$ by the following local transformations of the vielbein and the connections

$$
\begin{aligned}
\delta_{\mathcal{K}} E_{M}{ }^{A} & =\partial_{M} \xi^{A}+E_{M}^{C} \xi^{B} T_{B C}{ }^{A}+\omega_{M} \underline{c}^{B} f_{B \underline{c}}^{A}+E_{M}^{C} \Lambda^{\underline{b}} f_{\underline{b} C}{ }^{A} \\
\delta_{\mathcal{K}} \omega_{M} \underline{\underline{a}} & =\partial_{M} \Lambda^{\underline{a}}+E_{M}{ }^{C} \xi^{B} R_{B C^{\underline{a}}}+\omega_{M^{\underline{c}} \xi^{B}} f_{B \underline{\underline{c}}}^{\underline{a}}+E_{M}{ }^{C} \Lambda^{\underline{b}} f_{\underline{b} C^{\underline{a}}}+\omega_{M} M^{\underline{c}} \Lambda^{\underline{b}} f_{\underline{\underline{b}} \underline{\underline{a}}}^{\underline{a}}
\end{aligned}
$$

Here $\xi^{A}=\xi^{A}(z)$ parametrizes the covariant general coordinate transformations and $\Lambda^{\underline{a}}=$ $\Lambda^{\underline{a}}(z)=\left(\Lambda^{a b}, \Lambda^{i j}, \sigma, \Lambda_{\alpha}^{i}, \Lambda^{a}\right)$ are the gauge parameters associated with the structure group $\mathcal{H}$. It is important to observe that in (A.5) $f_{\underline{b c}} \underline{a}, f_{\underline{b} C^{\underline{a}}}$, and $f_{\underline{b} C} A$ are components of the structure constants of the superconformal algebra (A.1) and (A.3). The superfields $T_{B C}{ }^{A}$ and $R_{B C} \underline{a}$ are respectively the torsion and curvature tensors that appear as components of the two-forms

$$
\begin{aligned}
T^{C} & :=\frac{1}{2} E^{B} \wedge E^{A} T_{A B}^{C}=\mathrm{d} E^{C}-E^{B} \wedge \omega^{\underline{a}} f_{\underline{a} B}^{C} \\
R^{\underline{c}} & :=\frac{1}{2} E^{B} \wedge E^{A} R_{A B^{\underline{c}}}=\mathrm{d} \omega^{\underline{c}}-E^{B} \wedge \omega^{\underline{a}} f_{\underline{a}} B^{\underline{c}}-\frac{1}{2} \omega^{\underline{b}} \wedge \omega^{\underline{a}} f_{\underline{a b}}^{\underline{c}} .
\end{aligned}
$$

\footnotetext{
${ }^{20}$ Note that in the conventional superspace approach to $6 \mathrm{D}$ supergravity the locally superconformal structure is encoded in super-Weyl transformations $[26,66]$ analogously to the $4 \mathrm{D}$ case [18].
} 
From the explicit structure constants of the superconformal algebra, these tensors become

$$
\begin{aligned}
T^{a} & =\mathrm{d} E^{a}+E^{b} \wedge \Omega_{b}{ }^{a}+E^{a} \wedge B, \\
T_{i}^{\alpha} & =\mathrm{d} E_{i}^{\alpha}+E_{i}^{\beta} \wedge \Omega_{\beta}{ }^{\alpha}+\frac{1}{2} E_{i}^{\alpha} \wedge B-E^{\alpha j} \wedge \Phi_{j i}-\mathrm{i} E^{c} \wedge \mathfrak{F}_{\beta i}\left(\tilde{\gamma}_{c}\right)^{\alpha \beta}, \\
R(\mathbb{D}) & =\mathrm{d} B+2 E^{a} \wedge \mathfrak{F}_{a}+2 E_{i}^{\alpha} \wedge \mathfrak{F}_{\alpha}^{i}, \\
R(M)^{a b} & =\mathrm{d} \Omega^{a b}+\Omega^{a c} \wedge \Omega_{c}{ }^{b}-4 E^{[a} \wedge \mathfrak{F}^{b]}+2 E_{j}^{\alpha} \wedge \mathfrak{F}_{\beta}{ }^{j}\left(\gamma^{a b}\right)_{\alpha}{ }^{\beta}, \\
R(J)^{i j} & =\mathrm{d} \Phi^{i j}-\Phi^{k(i} \wedge \Phi^{j)}{ }_{k}-8 E^{\alpha(i} \wedge \mathfrak{F}_{\alpha}^{j)}, \\
R(K)^{a} & =\mathrm{d} \mathfrak{F}^{a}+\mathfrak{F}^{b} \wedge \Omega_{b}{ }^{a}-\mathfrak{F}^{a} \wedge B-\mathrm{i} \mathfrak{F}_{\alpha}^{k} \wedge \mathfrak{F}_{\beta k}\left(\tilde{\gamma}^{a}\right)^{\alpha \beta}, \\
R(S)_{\alpha}^{i} & =\mathrm{d} \mathfrak{F}_{\alpha}^{i}-\mathfrak{F}_{\beta}^{i} \wedge \Omega_{\alpha}{ }^{\beta}-\frac{1}{2} \mathfrak{F}_{\alpha}^{i} \wedge B-\mathfrak{F}_{\alpha}^{j} \wedge \Phi_{j}{ }^{i}-\mathrm{i} E^{\beta i} \wedge \mathfrak{F}^{c}\left(\gamma_{c}\right)_{\alpha \beta} .
\end{aligned}
$$

This gauging leads to a consistent modification of the superconformal algebra (A.3) described by the (anti)commutation relations

$$
\begin{aligned}
{\left[X_{\underline{a}}, X_{\underline{b}}\right\} } & =-f_{\underline{a b}} \underline{\underline{c}} X_{\underline{c}}, \\
{\left[X_{\underline{a}}, \nabla_{B}\right\} } & =-f_{\underline{a} B}{ }^{C} \nabla_{C}-f_{\underline{a}} B^{\underline{c}} X_{\underline{c}}, \\
{\left[\nabla_{A}, \nabla_{B}\right\} } & =-T_{A B}{ }^{C} \nabla_{C}-R_{A B} X_{\underline{c}},
\end{aligned}
$$

where the generators of the supertranslations, $P_{A}$, are replaced by the covariant derivatives $\nabla_{A}$. The $\mathcal{K}$ transformations, (A.5), can then be described by the following variation of the covariant derivatives

$$
\delta_{\mathcal{K}} \nabla_{A}=\left[\mathcal{K}, \nabla_{A}\right], \quad \mathcal{K}:=\xi^{C} \nabla_{C}+\Lambda^{\underline{c}} X_{\underline{c}}=\xi^{C} \nabla_{C}+\frac{1}{2} \Lambda^{c d} M_{c d}+\Lambda^{k l} J_{k l}+\sigma \mathbb{D}+\Lambda_{A} K^{A},
$$

provided that one interprets the action of the covariant derivatives on the parameters as

$$
\nabla_{A} \xi^{B}:=E_{A} \xi^{B}+\omega_{A} \underline{c}^{D} f_{D \underline{c}}^{B}, \quad \nabla_{A} \Lambda^{\underline{b}}:=E_{A} \Lambda^{\underline{b}}+\omega_{A} \underline{c}^{D} f_{D \underline{\underline{c}} \underline{\underline{b}}}+\omega_{A} \underline{\underline{c}} \Lambda^{\underline{d}} f_{\underline{d c}} \underline{\underline{b}} .
$$

A covariant superfield $\Phi$ is such that under $\mathcal{K}$ transformations it varies with no derivatives on the parameters and can be represented as

$$
\delta_{\mathcal{K}} \Phi=\mathcal{K} \Phi
$$

Due to (A.9), covariant derivatives of $\Phi$ transform covariantly $\delta_{\mathcal{K}}\left(\nabla_{A} \Phi\right)=\mathcal{K} \nabla_{A} \Phi$. The torsion and curvatures superfields, $T_{A B}{ }^{C}$ and $R_{A B}$, are necessarily covariant. A superfield $\Phi$ is said to be primary if it is annihilated by the special conformal generators, $K^{A} \Phi=0$. Due to (A.1), $S_{i}^{\alpha} \Phi=0$ is a sufficient condition for $\Phi$ to be primary.

In [9] it was proven that a consistent description of the $6 \mathrm{D} \mathcal{N}=(1,0)$ Weyl multiplet in conformal superspace can be achieved by:

i) choosing the gauging in conformal superspace described before that leads to the algebraic structures in (A.8); 
ii) requiring the covariant derivative algebra to resemble the one of $6 \mathrm{D} \mathcal{N}=(1,0)$ super Yang-Mills theory [54, 67-69], which takes the form

$$
\begin{aligned}
\left\{\nabla_{\alpha}^{i}, \nabla_{\beta}^{j}\right\} & =-2 \mathrm{i} \varepsilon^{i j}\left(\gamma^{a}\right)_{\alpha \beta} \nabla_{a}, \\
{\left[\nabla_{a}, \nabla_{\alpha}^{i}\right] } & =\left(\gamma_{a}\right)_{\alpha \beta} \mathcal{W}^{\beta i}, \\
{\left[\nabla_{a}, \nabla_{b}\right] } & =-\frac{\mathrm{i}}{8}\left(\gamma_{a b}\right)_{\alpha}{ }^{\beta}\left\{\nabla_{\beta}^{k}, \mathcal{W}_{k}^{\alpha}\right\},
\end{aligned}
$$

where $\mathcal{W}^{\alpha i}$ is a primary dimension $3 / 2$ operator such that

$$
\left[K^{A}, \mathcal{W}^{\alpha i}\right\}=0, \quad\left\{\nabla_{\alpha}^{(i}, \mathcal{W}^{\beta j)}\right\}=\frac{1}{4} \delta_{\alpha}^{\beta}\left\{\nabla_{\gamma}^{(i}, \mathcal{W}^{\gamma j)}\right\}, \quad\left\{\nabla_{\gamma}^{k}, \mathcal{W}_{k}^{\gamma}\right\}=0
$$

iii) constraining the operator $\mathcal{W}^{\alpha i}$ to be of the form

$$
\mathcal{W}^{\alpha i}=W^{\alpha \beta} \nabla_{\beta}^{i}+\frac{1}{2} \mathcal{W}(M)^{\alpha i a b} M_{a b}+\mathcal{W}(J)^{\alpha i j k} J_{j k}+\mathcal{W}(\mathbb{D})^{\alpha i} \mathbb{D}+\mathcal{W}(K)^{\alpha i}{ }_{B} K^{B}
$$

where $W^{\alpha \beta}=\frac{1}{3 !}\left(\tilde{\gamma}^{a b c}\right)^{\alpha \beta} W_{a b c}$ is the super-Weyl tensor [26, 70, 71] such that

$$
S_{k}^{\gamma} W^{\alpha \beta}=0, \quad \mathbb{D} W^{\alpha \beta}=W^{\alpha \beta} .
$$

It turns out that, under the previous assumptions, the super-Jacobi identities for the algebra (A.3) uniquely fix all the superfields $\mathcal{W}(M)^{\alpha i a b}, \mathcal{W}(J)^{\alpha i j k}, \mathcal{W}(\mathbb{D})^{\alpha i}, \mathcal{W}(K)^{\alpha i}{ }_{B}$ together with the torsion and curvatures in terms of $W^{\alpha \beta}$ and its covariant derivatives. Moreover, $W^{\alpha \beta}$ satisfies the Bianchi identities

$$
\begin{aligned}
\nabla_{\alpha}^{(i} \nabla_{\beta}^{j)} W^{\gamma \delta} & =-\delta_{[\alpha}^{(\gamma} \nabla_{\beta]}^{(i} \nabla_{\rho}^{j)} W^{\delta) \rho}, \\
\nabla_{\alpha}^{k} \nabla_{\gamma k} W^{\beta \gamma}-\frac{1}{4} \delta_{\alpha}^{\beta} \nabla_{\gamma}^{k} \nabla_{\delta k} W^{\gamma \delta} & =8 \mathrm{i} \nabla_{\alpha \gamma} W^{\gamma \beta}
\end{aligned}
$$

We refer the reader to [9] for more details.

\section{A.3 Different superspace frames}

It is worth underlining that the action of the generators $X_{\underline{a}}$ on $\nabla_{A}$, eq. (A.8b), was chosen in [9] to be identical to the action on $P_{A}$, eq. (A.3). This condition leads for example to a simple choice (A.12a) for the form of the supersymmetry algebra, but gives more complicated constraints on the vector curvatures (2.16), which contribute a number of covariant fields into the composite connections. As described in the main body of the paper, for components applications, different choices of conventional constraints can be more convenient. In conformal superspace this results in a framework where the structure constants $f_{\underline{a}} B^{\underline{c}}$ are replaced by structure functions $\hat{f}_{\underline{a}} B^{\underline{c}} \cdot{ }^{21}$

\footnotetext{
${ }^{21}$ The reader can find a pedagogical discussion of structure functions in the textbook [33].
} 
For applications in this paper we use a change of frame where only the vector covariant derivatives are modified. In particular, we introduce the $\hat{\nabla}_{A}$ derivatives as ${ }^{22}$

$$
\begin{aligned}
\hat{\nabla}_{\alpha}^{i}:= & \nabla_{\alpha}^{i}, \\
\hat{\nabla}_{a}:= & \nabla_{a}-\frac{1}{2} \lambda_{1} W_{a}^{b c} M_{b c}-\mathrm{i} \lambda_{2}\left(\gamma_{a}\right)_{\alpha \beta} X^{\alpha j} S_{j}^{\beta} \\
& -\left(\lambda_{3} Y \eta_{a c}+\lambda_{4} \nabla^{b} W_{a b c}+\lambda_{5} W_{a}^{e f} W_{e f c}\right) K^{c},
\end{aligned}
$$

where $\lambda_{1}, \lambda_{2}, \lambda_{3}, \lambda_{4}, \lambda_{5}$ are arbitrary real constant parameters and the dimension $3 / 2$ covariant superfield $X_{i}^{\alpha}$ is defined as

$$
X^{\alpha i}:=-\frac{\mathrm{i}}{10} \nabla_{\beta}^{i} W^{\alpha \beta} .
$$

The new $\hat{\nabla}_{A}$ derivatives have the same vielbein of $\nabla_{A}, E_{M}{ }^{A}$, but have modified connections

$$
\hat{\nabla}_{A}:=E_{A}^{M}\left(\partial_{M}-\hat{\omega}_{M} \underline{a}^{\underline{a}}\right), \quad \hat{\omega}_{M^{\underline{a}}}=\omega_{M^{\underline{a}}}+E_{M}{ }^{B} \mathcal{M}_{B^{\underline{a}}}^{\underline{a}} .
$$

For the change of frame (A.17), $\mathcal{M}_{A} \underline{c}$ is given by

$$
\begin{aligned}
\mathcal{M}_{a}^{c d} & =\lambda_{1} W_{a}^{c d} \\
\mathcal{M}_{a \gamma}^{k} & =-\mathrm{i} \lambda_{2}\left(\gamma_{a}\right)_{\gamma \delta} X^{\delta k} \\
\mathcal{M}_{a c} & =\lambda_{3} Y \eta_{a c}+\lambda_{4} \nabla^{b} W_{a b c}+\lambda_{5} W_{a}{ }^{e f} W_{e f c}
\end{aligned}
$$

with all the other components identically zero.

Given the algebra (A.8) of $\left(X_{a}, \nabla_{A}\right)$ and the relations (A.17), it is straightforward to show that the (anti)commutation relations satisfied by $X_{\underline{a}}$ and $\hat{\nabla}_{A}$ have the following form

$$
\begin{aligned}
{\left[X_{\underline{a}}, X_{\underline{b}}\right\} } & =-f_{\underline{a b}} \underline{\underline{c}} X_{\underline{c}}, \\
{\left[X_{\underline{a}}, \hat{\nabla}_{B}\right\} } & =-f_{\underline{a} B}{ }^{C} \hat{\nabla}_{C}-\hat{f}_{\underline{a}} B^{\underline{c}} X_{\underline{c}}, \\
{\left[\hat{\nabla}_{A}, \hat{\nabla}_{B}\right\} } & =-\hat{T}_{A B} C \hat{\nabla}_{C}-\hat{R}_{A B} X_{\underline{c}} .
\end{aligned}
$$

Here $f_{\underline{a} \underline{\underline{a}}}, f_{\underline{a} B}^{C}$ match the structure constants of the superconformal group, but $f_{\underline{a}} A^{\underline{c}}$ becomes a nontrivial structure function $\hat{f}_{\underline{a}} A^{\underline{c}}$ that has dependence on $W_{a b c}$ and its descendant superfields. In the new frame, the torsion and curvatures, $\hat{T}_{A B}{ }^{C}$ and $\hat{R}_{A B^{\underline{c}}}$, have the same form as their unhatted partners in (A.6), with $\omega_{\underline{a}} \rightarrow \hat{\omega}_{\underline{a}}$ and $f_{\underline{a}} B^{\underline{c}} \rightarrow \hat{f}_{\underline{a}} B^{\underline{c}}$.

It turns out that by properly tuning the parameters $\lambda_{1}$ and $\lambda_{4}$ as

$$
\lambda_{1}=2, \quad \lambda_{4}=-\frac{1}{2}
$$

we can set to zero the torsion $\hat{T}_{a b}{ }^{c}$ and the dilatation curvature

$$
\hat{T}_{a b}{ }^{c}=0, \quad \hat{R}(\mathbb{D})_{a b}=0 .
$$

\footnotetext{
${ }^{22}$ Note that, due to $W_{a[b}{ }^{e} W_{c d] e}=0$ the useful relation $\nabla_{a} W_{b c d}=\hat{\nabla}_{a} W_{b c d}$ holds.
} 
Moreover, the choice of parameters (A.22) removes terms of the form $\hat{\nabla}^{c} W_{a b c}$ from $\hat{R}_{a b}{ }^{c d}(M)$ and ensures that $\hat{f}^{a} b^{\underline{c}}=f^{a} b^{\underline{c}}$, so that

$$
\left[K_{a}, \hat{\nabla}_{b}\right]=2 \eta_{a b} \mathbb{D}+2 M_{a b}
$$

The choice (A.22) simplifies the component analysis and we will assume it from now on. We leave $\lambda_{2}, \lambda_{3}$ and $\lambda_{5}$ unfixed for the moment although two sets of choices, highlighted in table 1, are particularly interesting. The first, that we denoted as "Traceless," gives rise to a superspace geometry whose projection to components, as described in section 2.3, leads to convenient constraints on the component curvatures. The second choice leads to a superspace whose component constraints are identical to the ones originally used in [30].

With the choice (A.22), the structure constants $\hat{f}_{\underline{a}} A^{\underline{c}}$ turn out to have the following nontrivial components

$$
\begin{aligned}
\hat{f}_{i b \gamma}^{\alpha k} & =\left(\frac{1}{2}+\frac{8}{5} \lambda_{2}\right) W_{b c d} \delta_{i}^{k}\left(\tilde{\gamma}^{c d}\right)^{\alpha}{ }_{\gamma} \\
\hat{f}_{i b c}^{\alpha} & =-2\left(\lambda_{2}+2 \lambda_{3}\right) X_{i}^{\alpha} \eta_{b c}-\left(1+2 \lambda_{2}\right)\left(\tilde{\gamma}_{b c}\right)^{\alpha}{ }_{\beta} X_{i}^{\beta}-\frac{1}{2}\left(\gamma_{b c}\right)_{\beta}{ }^{\gamma} X_{\gamma i}{ }^{\beta \alpha}
\end{aligned}
$$

while all the other components of $\hat{f}_{\underline{a}} b^{\underline{c}}$ are identical to the ones of the superconformal algebra. Here the dimension $3 / 2$ covariant superfield $X_{\gamma}^{k \beta \alpha}$ is [9]

$$
X_{\gamma}^{k \alpha \beta}=-\frac{\mathrm{i}}{4} \nabla_{\gamma}^{k} W^{\alpha \beta}-\delta_{\gamma}^{(\alpha} X^{\beta) k}, \quad X_{\gamma}^{k \alpha \beta}=X_{\gamma}^{k \beta \alpha}, \quad X_{\gamma}^{k \alpha \gamma}=0
$$

Note that for the choice of frame we are considering, the commutator $\left[S_{i}^{\alpha}, \hat{\nabla}_{b}\right]$ is

$$
\begin{aligned}
{\left[S_{i}^{\alpha}, \hat{\nabla}_{b}\right]=} & -\mathrm{i}\left(\tilde{\gamma}_{b}\right)^{\alpha \beta} \hat{\nabla}_{\beta i}-\left(\frac{1}{2}+\frac{8}{5} \lambda_{2}\right) W_{b c d}\left(\tilde{\gamma}^{c d}\right)^{\alpha}{ }_{\gamma} S_{i}^{\gamma}+2\left(\lambda_{2}+2 \lambda_{3}\right) X_{i}^{\alpha} K_{b} \\
& +\left[\left(1+2 \lambda_{2}\right)\left(\tilde{\gamma}_{b c}\right)^{\alpha}{ }_{\beta} X_{i}^{\beta}+\frac{1}{2}\left(\gamma_{b c}\right)_{\beta}{ }^{\gamma} X_{\gamma i}{ }^{\beta \alpha}\right] K^{c}
\end{aligned}
$$

The torsion $\hat{T}_{A B}{ }^{C}$ and curvatures $\hat{R}_{A B^{\underline{c}}}$ in the new frame can be computed by using the (anti)commutation relations of the $\nabla_{A}$ derivatives derived in [9] together with (A.17). The anticommutator of two spinor derivatives, $\left\{\hat{\nabla}_{\alpha}^{i}, \hat{\nabla}_{\beta}^{j}\right\}$, has the following torsion and curvatures

$$
\begin{aligned}
\hat{T}_{\alpha \beta}^{i j c} & =2 \mathrm{i} \varepsilon^{i j}\left(\gamma^{c}\right)_{\alpha \beta} \\
\hat{R}(M)_{\alpha \beta}^{i j j c d} & =4 \mathrm{i} \varepsilon^{i j}\left(\gamma_{a}\right)_{\alpha \beta} W^{a c d} \\
\hat{R}(S)_{\alpha \beta \gamma}^{i j k} & =4 \lambda_{2} \varepsilon^{i j} \varepsilon_{\alpha \beta \gamma \delta} X^{\delta k} \\
\hat{R}(K)_{\alpha \beta c}^{i j} & =2 \mathrm{i} \varepsilon^{i j}\left(\gamma^{a}\right)_{\alpha \beta}\left(\lambda_{3} \eta_{a c} Y+\lambda_{4} \hat{\nabla}^{b} W_{a b c}+\lambda_{5} W_{a}{ }^{e f} W_{c e f}\right),
\end{aligned}
$$


where the omitted components vanish. The non-zero torsion and curvatures in the commutator $\left[\hat{\nabla}_{a}, \hat{\nabla}_{\beta}^{j}\right]$ are:

$$
\begin{aligned}
& \hat{T}_{a \beta k}^{j \gamma}=-\frac{1}{2}\left(\gamma_{a}\right)_{\beta \delta} W^{\delta \gamma} \delta_{k}^{j}, \\
& \hat{R}(\mathbb{D})_{a_{\beta}}^{j}=-2 \mathrm{i}\left(\lambda_{2}+\frac{5}{8}\right)\left(\gamma_{a}\right)_{\beta \gamma} X^{\gamma j} \\
& \hat{R}(M)_{a_{\beta}}^{j c d}=-2 \mathrm{i}\left(\lambda_{2}+\frac{3}{8}\right)\left(\gamma_{a}^{c d}\right)_{\beta \gamma} X^{\gamma j}-4 \mathrm{i}\left(\lambda_{2}+\frac{1}{8}\right) \delta_{a}^{[c}\left(\gamma^{d]}\right)_{\beta \gamma} X^{\gamma j} \\
&-\mathrm{i}\left(\gamma_{a}^{c d}\right)_{\gamma \delta} X_{\beta}^{j \gamma \delta}+2 \mathrm{i}\left(\gamma_{a}\right)_{\beta \gamma}\left(\gamma^{c d}\right)_{\delta}^{\rho} X_{\rho}^{j \gamma \delta} \\
& \hat{R}(J)_{a_{\beta}}^{j k l}= 8 \mathrm{i}\left(\lambda_{2}+\frac{5}{8}\right)\left(\gamma_{a}\right)_{\beta \gamma} X^{\gamma(k} \varepsilon^{l) j} \\
& \hat{R}(S)_{a_{\beta \gamma}}^{j k}= \mathrm{i}\left(\frac{5}{16}+\frac{1}{2} \lambda_{2}-\lambda_{3}\right)\left(\gamma_{a}\right)_{\beta \gamma} \varepsilon^{j k} Y-\frac{\mathrm{i}}{4}\left(\gamma_{a}\right)_{\beta \delta} Y_{\gamma}^{\delta j k}-\frac{2 \mathrm{i}}{5} \lambda_{2}\left(\gamma_{a}\right)_{\gamma \delta} Y_{\beta}{ }^{\delta j k} \\
&-\frac{\mathrm{i}}{8}\left(\gamma_{a}\right)_{\beta \delta} \hat{\nabla}_{\gamma \rho} W^{\delta \rho} \varepsilon^{j k}-\mathrm{i}\left(\frac{1}{8}+\frac{2}{5} \lambda_{2}\right)\left(\gamma_{a}\right)_{\gamma \delta} \hat{\nabla}_{\beta \rho} W^{\delta \rho} \varepsilon^{j k} \\
&-\frac{\mathrm{i}}{4} \lambda_{5}\left(\gamma_{a}\right)_{\delta \epsilon} \varepsilon_{\beta \rho \tau \gamma} W^{\delta \rho} W^{\epsilon \tau} \varepsilon^{j k}, \\
& \frac{\mathrm{i}}{4}\left(\gamma_{c}\right)_{\beta \gamma} \hat{\nabla}_{a} X^{\gamma j}+2 \mathrm{i}\left(\lambda_{3}-\frac{1}{8}\right) \eta_{a c} \hat{\nabla}_{\beta \gamma} X^{\gamma j}-\frac{\mathrm{i}}{4}\left(\gamma_{a c d}\right)_{\gamma \delta} \hat{\nabla}^{d} X_{\beta}^{j \gamma \delta} \\
&+\frac{\mathrm{i}}{3}\left(\gamma_{a}\right)_{\beta \delta}\left(\gamma_{c d}\right)_{\rho}^{\gamma} \hat{\nabla}^{d} X_{\gamma}^{j \delta \rho}+\mathrm{i}\left(\lambda_{2}+\frac{1}{2} \lambda_{5}+\frac{1}{8}\right)\left(\gamma_{a}\right)_{\delta \rho}\left(\gamma_{c}\right)_{\beta \gamma} W^{\gamma \delta} X^{\rho j} \\
&+\mathrm{i}\left(\lambda_{2}+\frac{1}{2} \lambda_{5}\right)\left(\gamma_{a}\right)_{\beta \gamma}\left(\gamma_{c}\right)_{\delta \rho} W^{\gamma \delta} X^{\rho j}+\frac{5 \mathrm{i}}{12}\left(\gamma_{a}\right)_{\beta \rho}\left(\gamma_{c}\right)_{\gamma \epsilon} W^{\gamma \delta} X_{\delta}^{j \rho \epsilon} \\
&+\frac{\mathrm{i}}{4}\left(\gamma_{a}\right)_{\gamma \rho}\left(\gamma_{c}\right)_{\beta \epsilon} W^{\gamma \delta} X_{\delta}^{j \rho \epsilon}-\mathrm{i} \lambda_{5}\left(\gamma_{a}\right)_{\gamma \rho}\left(\gamma_{c}\right)_{\delta \epsilon} W^{\gamma \delta} X_{\beta}^{j \rho \epsilon} \\
& a_{\beta c}
\end{aligned}
$$

In the new frame, the commutator of two vector derivatives, $\left[\hat{\nabla}_{a}, \hat{\nabla}_{b}\right]$, has the following non-vanishing torsion and curvatures:

$$
\begin{aligned}
\hat{T}_{a b k}^{\gamma}= & \left(\gamma_{a b}\right)_{\beta}{ }^{\alpha}\left[X_{\alpha k}^{\beta \gamma}-2\left(\lambda_{2}+\frac{3}{8}\right) \delta_{\alpha}^{\gamma} X_{k}^{\beta}\right] \\
\hat{R}(M)_{a b}{ }^{c d}= & Y_{a b}{ }^{c d}+8\left(\lambda_{3}-\frac{1}{8}\right) Y \delta_{a}^{[c} \delta_{b}^{d]}+8\left(\lambda_{5}-\frac{1}{2}\right) W_{a b f} W^{f c d}, \\
\hat{R}(J)_{a b}{ }^{k l}= & \frac{1}{2}\left(\gamma_{a b}\right)_{\delta}{ }^{\gamma} Y_{\gamma}^{\delta k l}=Y_{a b}{ }^{k l} \\
\hat{R}(S)_{a b \gamma}{ }^{k}= & -\frac{\mathrm{i}}{3}\left(\gamma_{a b}\right)_{\delta}{ }^{\alpha} \hat{\nabla}_{\gamma \beta} X_{\alpha}^{k \beta \delta}-\frac{\mathrm{i}}{6}\left(\gamma_{a b c}\right)_{\alpha \beta} \hat{\nabla}^{c} X_{\gamma}^{k \alpha \beta}-\frac{\mathrm{i}}{6} \varepsilon_{\gamma \beta \epsilon \rho}\left(\gamma_{a b}\right)_{\delta}{ }^{\rho} W^{\alpha \beta} X_{\alpha}^{k \delta \epsilon} \\
& +\mathrm{i}\left(2 \lambda_{2}+\frac{3}{4}\right) \hat{\nabla}_{[a} X^{\alpha k}\left(\gamma_{b]}\right)_{\alpha \gamma}-\frac{\mathrm{i}}{2}\left(\lambda_{2}+\frac{3}{8}\right) \varepsilon_{\gamma \beta \delta \epsilon}\left(\gamma_{a b}\right)_{\alpha}{ }^{\epsilon} W^{\alpha \beta} X^{\delta k},
\end{aligned}
$$




$$
\begin{aligned}
\hat{R}(K)_{a b c}= & \frac{1}{4} \hat{\nabla}^{d} Y_{a b c d}+\frac{\mathrm{i}}{3} X_{\alpha}^{k \beta \gamma} X_{\beta k}{ }^{\alpha \delta}\left(\gamma_{a b c}\right)_{\gamma \delta}+\mathrm{i}\left(\gamma_{a b}\right)_{\epsilon}{ }^{\alpha}\left(\gamma_{c}\right)_{\gamma \delta} X_{\alpha}^{k \beta \gamma} X_{\beta k}{ }^{\delta \epsilon} \\
& +\frac{5 \mathrm{i}}{3}\left(\lambda_{2}+\frac{3}{8}\right) X^{\gamma k} X_{\gamma k}{ }^{\alpha \beta}\left(\gamma_{a b c}\right)_{\alpha \beta}-4 \mathrm{i}\left(\lambda_{2}+\frac{5}{16}\right) X^{\alpha k} X_{\beta k}{ }^{\gamma \delta}\left(\gamma_{a b}\right)_{\gamma}{ }^{\beta}\left(\gamma_{c}\right)_{\alpha \delta} \\
& +2 \mathrm{i}\left(\lambda_{2}+\frac{3}{8}\right)\left(\lambda_{2}+\frac{5}{8}\right) X^{\alpha k} X_{k}^{\beta}\left(\gamma_{a b c}\right)_{\alpha \beta}+2\left(\lambda_{3}-\frac{1}{8}\right) \hat{\nabla}_{[a} Y \eta_{b] c} \\
& +\frac{1}{2}\left(\lambda_{5}-\frac{1}{2}\right) W^{\alpha \beta} \hat{\nabla}_{[a} W^{\gamma \delta}\left(\gamma_{b]}\right)_{\alpha \gamma}\left(\gamma_{c}\right)_{\beta \delta}
\end{aligned}
$$

Note that we have introduced the following higher dimension descendant superfields constructed from spinor derivatives of $W^{\alpha \beta}$ :

$$
\begin{aligned}
Y_{\alpha}^{\beta i j} & :=-\frac{5}{2}\left(\nabla_{\alpha}^{(i} X^{\beta j)}-\frac{1}{4} \delta_{\alpha}^{\beta} \nabla_{\gamma}^{(i} X^{\gamma j)}\right), \\
Y & :=\frac{1}{4} \nabla_{\gamma}^{k} X_{k}^{\gamma}, \\
Y_{\alpha \beta}{ }^{\gamma \delta} & :=\nabla_{(\alpha}^{k} X_{\beta) k}^{\gamma \delta}-\frac{1}{3} \nabla_{\rho}^{k} X_{(\alpha k}{ }^{\rho(\gamma} \delta_{\beta)}^{\delta)} .
\end{aligned}
$$

By using (A.16) and the previous definitions, one can derive the following relations for the descendant superfields:

$$
\begin{aligned}
& \nabla_{\alpha}^{i} X^{\beta j}=-\frac{2}{5} Y_{\alpha}^{\beta i j}-\frac{2}{5} \varepsilon^{i j} \hat{\nabla}_{\alpha \gamma} W^{\gamma \beta}-\frac{1}{2} \varepsilon^{i j} \delta_{\alpha}^{\beta} Y, \\
& \nabla_{\alpha}^{i} X_{\beta}^{j \gamma \delta}=\frac{1}{2} \delta_{\alpha}^{(\gamma} Y_{\beta}^{\delta) i j}-\frac{1}{10} \delta_{\beta}^{(\gamma} Y_{\alpha}{ }^{\delta) i j}-\frac{1}{2} \varepsilon^{i j} Y_{\alpha \beta}{ }^{\gamma \delta}-\frac{1}{4} \varepsilon^{i j} \hat{\nabla}_{\alpha \beta} W^{\gamma \delta} \\
& +\frac{3}{20} \varepsilon^{i j} \delta_{\beta}^{(\gamma} \hat{\nabla}_{\alpha \rho} W^{\delta) \rho}-\frac{1}{4} \varepsilon^{i j} \delta_{\alpha}^{(\gamma} \hat{\nabla}_{\beta \rho} W^{\delta) \rho}, \\
& \nabla_{\alpha}^{i} Y=-2 \mathrm{i} \hat{\nabla}_{\alpha \beta} X^{\beta i} \\
& \nabla_{\gamma}^{k} Y_{\alpha}{ }^{\beta i j}=\frac{2}{3} \varepsilon^{k(i}\left(-8 \mathrm{i} \hat{\nabla}_{\gamma \delta} X_{\alpha}^{j) \beta \delta}-4 \mathrm{i} \hat{\nabla}_{\alpha \delta} X_{\gamma}^{j) \beta \delta}+3 \mathrm{i} \hat{\nabla}_{\gamma \alpha} X^{\beta j)}+3 \mathrm{i} \delta_{\gamma}^{\beta} \hat{\nabla}_{\alpha \delta} X^{\delta j)}\right. \\
& \left.-\frac{3 \mathrm{i}}{2} \delta_{\alpha}^{\beta} \hat{\nabla}_{\gamma \delta} X^{\delta j)}-3 \mathrm{i} \varepsilon_{\alpha \gamma \delta \epsilon} W^{\beta \delta} X^{\epsilon j)}+4 \mathrm{i} \varepsilon_{\alpha \gamma \epsilon \rho} W^{\delta \epsilon} X_{\delta}^{j) \beta \rho}\right), \\
& \left.\left.\nabla_{\epsilon}^{l} Y_{\alpha \beta}{ }^{\gamma \delta}=-4 \mathrm{i} \hat{\nabla}_{\epsilon(\alpha} X_{\beta)}^{l}{ }^{\gamma \delta}+\frac{4 \mathrm{i}}{3} \delta_{(\alpha}^{(\gamma} \hat{\nabla}_{\beta) \rho} X_{\epsilon}^{l \delta) \rho}+\frac{8 \mathrm{i}}{3} \delta_{(\alpha}^{(\gamma} \hat{\nabla}_{|\epsilon \rho|} X_{\beta)}^{l} \delta\right) \rho+8 \mathrm{i} \delta_{\epsilon}^{(\gamma} \hat{\nabla}_{\rho(\alpha} X_{\beta)}^{l} \delta\right) \rho \\
& \left.-\frac{4 \mathrm{i}}{3} W^{\rho \sigma} \delta_{(\alpha}^{(\gamma} \varepsilon_{\beta) \epsilon \sigma \tau} X_{\rho}^{l \delta) \tau}-8 \mathrm{i} \varepsilon_{\epsilon \rho \sigma(\alpha} W^{\rho(\gamma} X_{\beta)}^{l} \delta\right) \sigma .
\end{aligned}
$$

These relations define the $Q$-supersymmetry transformations of the descendant superfields of the super-Weyl tensor. Their $S$-supersymmetry transformations are instead given by the following relations [9]:

$$
\begin{aligned}
& S_{i}^{\alpha} X^{\beta j}=\frac{8 \mathrm{i}}{5} \delta_{i}^{j} W^{\alpha \beta}, \quad S_{i}^{\alpha} X_{\beta}^{j \gamma \delta}=-\mathrm{i} \delta_{i}^{j} \delta_{\beta}^{\alpha} W^{\gamma \delta}+\frac{2 \mathrm{i}}{5} \delta_{i}^{j} \delta_{\beta}^{(\gamma} W^{\delta) \alpha}, \\
& S_{k}^{\gamma} Y_{\alpha}^{\beta i j}=-\delta_{k}^{(i}\left(16 X_{\alpha}^{j) \gamma \beta}-2 \delta_{\alpha}^{\beta} X^{\gamma j)}+8 \delta_{\alpha}^{\gamma} X^{\beta j)}\right), \\
& S_{j}^{\rho} Y_{\alpha \beta}{ }^{\gamma \delta}=24\left(\delta_{(\alpha}^{\rho} X_{\beta) j}{ }^{\gamma \delta}-\frac{1}{3} \delta_{(\alpha}^{(\gamma} X_{\beta) j}{ }^{\delta) \rho}\right), \quad S_{i}^{\alpha} Y=-4 X_{i}^{\alpha} .
\end{aligned}
$$


Note that the Bianchi identities for the $\hat{\nabla}_{A}$ derivatives are identically satisfied due to (A.16), (A.32) and the following useful relations

$$
\begin{aligned}
\hat{\nabla}_{\gamma(\alpha} Y_{\beta)}{ }^{\gamma i j}= & 0, \quad \hat{\nabla}^{\gamma(\alpha} Y_{\gamma}^{\beta) i j}=4 \mathrm{i}\left(5+8 \lambda_{2}\right) X^{\gamma(i} X_{\gamma}^{j) \alpha \beta} \\
\hat{\nabla}^{\delta(\alpha} X_{\delta}^{i \beta \gamma)}= & W^{\delta(\alpha} X_{\delta}^{i \beta \gamma)}+6\left(\lambda_{2}+\frac{3}{8}\right) W^{(\alpha \beta} X^{\gamma) i} \\
\hat{\nabla}_{\delta(\alpha} Y_{\beta \gamma)} \delta \epsilon= & 0, \\
\hat{\nabla}^{\delta(\alpha} Y_{\delta \epsilon}^{\beta \gamma)}= & 24 \mathrm{i} X_{\epsilon}^{k \delta(\alpha} X_{\delta k}^{\beta \gamma)}-8 \mathrm{i} X_{\rho}^{k \delta(\alpha} \delta_{\epsilon}^{\beta} X_{\delta k}^{\gamma) \rho}+96 \mathrm{i}\left(\lambda_{2}+\frac{3}{8}\right) X^{(\alpha k} X_{\epsilon k}{ }^{\beta \gamma)} \\
& -16 \mathrm{i}\left(\lambda_{2}+\frac{3}{8}\right) X^{\delta k} X_{\delta k}{ }^{(\beta \gamma} \delta_{\epsilon}^{\alpha)} .
\end{aligned}
$$

We conclude this appendix by mentioning that in the new frame the supergravity gauge transformations of the vielbein, the connections and of a covariant tensor superfield $\Phi$, respectively, are (compare with (A.5) and (A.11))

$$
\begin{aligned}
& \delta_{\mathcal{K}} E_{M}{ }^{A}=\partial_{M} \xi^{A}+E_{M}^{C} \xi^{B} \hat{T}_{B C}{ }^{A}+\hat{\omega}_{M}{ }^{\underline{c}} \xi^{B} f_{B \underline{c}}{ }^{A}+E_{M}{ }^{C} \hat{\Lambda}^{\underline{b}} f_{\underline{b} C}{ }^{A}, \\
& \delta_{\mathcal{K}} \hat{\omega}_{M}^{\underline{a}}=\partial_{M} \hat{\Lambda}^{\underline{a}}+E_{M}^{C} \xi^{B} \hat{R}_{B C^{\underline{a}}}+\hat{\omega}_{M} M^{\underline{c}} \xi^{B} \hat{f}_{B \underline{\underline{c}}} \underline{\underline{a}}+E_{M}{ }^{C} \hat{\Lambda}^{\underline{b}} \hat{f}_{\underline{b}} C^{\underline{a}}+\hat{\omega}_{M}{ }^{\underline{c}} \hat{\Lambda}^{\underline{b}} f_{\underline{b} \underline{\underline{c}}}{ }^{\underline{a}}, \\
& \delta_{\mathcal{K}} \Phi=\mathcal{K} \Phi,
\end{aligned}
$$

where the operator $\mathcal{K}$ is $\mathcal{K}=\left(\xi^{A} \hat{\nabla}_{A}+\hat{\Lambda}^{\underline{a}} X_{\underline{a}}\right)$ and the gauge parameters $\hat{\Lambda}^{\underline{a}}$ and $\Lambda^{\underline{a}}$ are related to each other by

$$
\hat{\Lambda}^{\underline{a}}=\Lambda^{\underline{a}}+\xi^{A} \mathcal{M}_{A}^{\underline{a}}
$$

\section{B Relating notation and conventions}

As underlined in section 2 and appendix A, the "hat" frame described in section 2.3 is equivalent to the one employed originally in [30] by choosing the parameters as follows

$$
\lambda_{1}=2, \quad \lambda_{2}=-\frac{5}{16}, \quad \lambda_{3}=\frac{5}{32}, \quad \lambda_{4}=-\frac{1}{2}, \quad \lambda_{5}=1
$$

This is true up to a choice of notation and conventions. In this appendix we describe the relevant notational differences and show how to obtain the results of [30] from the ones in section 2.3 .

First of all, note that throughout our paper we have used chiral four-component spinor notation while in [30] eight-component spinor notation is used. To match the results, one should first reinterpret our formulae using eight component spinors. This is straightforward by using appendix A of [9] where we refer the reader for more details. Our $8 \times 8$ Dirac spinors $\Psi$ and matrices $\Gamma^{a}$ are

$$
\Psi=\left(\begin{array}{c}
\psi^{\alpha} \\
\chi_{\alpha}
\end{array}\right), \quad \Gamma^{a}=\left(\begin{array}{cc}
0 & \left(\tilde{\gamma}^{a}\right)^{\alpha \beta} \\
\left(\gamma^{a}\right)_{\alpha \beta} & 0
\end{array}\right), \quad \Gamma_{*}=\left(\begin{array}{cc}
\delta_{\beta}^{\alpha} & 0 \\
0 & -\delta_{\alpha}^{\beta}
\end{array}\right),
$$

where $\Gamma_{*}$ obeys $\Gamma_{[a} \Gamma_{b} \Gamma_{c} \Gamma_{d} \Gamma_{e} \Gamma_{f]}=\varepsilon_{a b c d e f} \Gamma_{*}$. Similarly, there is a direct relation between $\gamma^{a_{1} \cdots a_{n}}, \tilde{\gamma}^{a_{1} \cdots a_{n}}$ and $\Gamma^{a_{1} \cdots a_{n}}:=\Gamma^{\left[a_{1}\right.} \Gamma^{a_{2}} \cdots \Gamma^{\left.a_{n}\right]}$ since a product of chiral $\gamma \mathrm{s}$ are straightforwardly lifted to a product of Dirac $\Gamma$ s. The eight component spinor generators of the $6 \mathrm{D}$ 


\begin{tabular}{|cc|}
\hline Our notation & Bergshoeff et al. [30] \\
\hline$\eta^{a b}, \varepsilon^{a b c d e f}, \Gamma^{a}, \Gamma^{a_{1} \cdots a_{n}}, \cdots$ & $\eta^{a b}, \varepsilon^{a b c d e f},-\mathrm{i} \gamma^{a},(-\mathrm{i})^{n} \gamma^{a_{1} \cdots a_{n}}, \cdots$ \\
\hline$P_{a}, K^{a}, Q^{i}, S_{i}$ & $P_{a}, K^{a},-2 Q^{i}, 2 S_{i}$ \\
$M_{a b}, J_{i j}, \mathbb{D}$ & $-2 M_{a b}, 2 U_{i j}, D$ \\
\hline$e_{m}{ }^{a}, \hat{\mathfrak{f}}_{m}{ }^{\hat{a}}, \psi_{m}{ }^{i}, \hat{\phi}_{m}{ }^{i}$ & $e_{\mu}{ }^{a}, f_{\mu}{ }^{a}, \psi_{\mu}{ }^{i}, \phi_{\mu}{ }^{i}$ \\
$\hat{\omega}_{m}{ }^{a b}, \mathcal{V}_{m}{ }^{i j}, b_{m}$ & $-\omega_{\mu}{ }^{a b}, \frac{1}{2} V_{\mu}{ }^{i j}, b_{\mu}$ \\
\hline$\hat{R}(P)_{a b}{ }^{c}, \hat{R}(K)_{a b c}, \hat{R}(Q)_{a b}{ }^{i}, \hat{R}(S)_{a b i}$ & $\hat{R}(P)_{a b}{ }^{c}, \hat{R}(K)_{a b c}, \frac{1}{2} \hat{R}(Q)_{a b}{ }^{i}, \frac{1}{2} \hat{R}(S)_{a b i}$ \\
$\hat{R}(M)_{a b}{ }^{c d}, \hat{R}(J)_{a b}{ }^{i j}, \hat{R}(\mathbb{D})_{a b}$, & $-\hat{R}(M)_{a b}{ }^{c d}, \frac{1}{2} \hat{R}(U)_{a b}{ }^{i j}, \hat{R}(\mathbb{D})_{a b}$ \\
\hline$\xi^{a}, \lambda^{a}, \xi_{i}, \eta^{i}$ & $\xi^{a}, \Lambda_{K}^{a}, \frac{1}{2} \varepsilon_{i}, \frac{1}{2} \eta^{i}$ \\
$\lambda^{a b}, \lambda^{i j}, \sigma$ & $-\varepsilon^{a b}, \frac{1}{2} \Lambda^{i j}, \Lambda_{D}$ \\
\hline
\end{tabular}

Table 2. Translation of notation and conventions.

$\mathcal{N}=(1,0)$ superconformal algebra are

$$
Q^{i}=\left(\begin{array}{c}
0 \\
Q_{\alpha}^{i}
\end{array}\right), \quad S_{i}=\left(\begin{array}{c}
S_{i}^{\alpha} \\
0
\end{array}\right) .
$$

Similarly, all the (anti)chiral spinor fields are straightforwardly lifted to eight components, such that, e.g. $\psi_{m_{i}}^{\alpha} Q_{\alpha}^{i} \rightarrow \bar{\psi}_{m i} Q^{i}, \hat{\phi}_{m}{ }^{i} S_{i}^{\alpha} \rightarrow \overline{\hat{\phi}}_{m}{ }^{i} S_{i}$. The results of [30] for the $6 \mathrm{D} \mathcal{N}=(1,0)$ Weyl multiplet may be obtained from the results of section 2 by lifting to eight-component spinors, fixing the parameters as in (B.1) and renaming the fields in accordance with table 2. We have normalized the covariant fields $T_{a b c}^{-}, \chi^{i}$ and $D$ to match those of [30].

Open Access. This article is distributed under the terms of the Creative Commons Attribution License (CC-BY 4.0), which permits any use, distribution and reproduction in any medium, provided the original author(s) and source are credited.

\section{References}

[1] S. Deser and A. Schwimmer, Geometric classification of conformal anomalies in arbitrary dimensions, Phys. Lett. B 309 (1993) 279 [hep-th/9302047] [INSPIRE].

[2] W. Nahm, Supersymmetries and their Representations, Nucl. Phys. B 135 (1978) 149 [INSPIRE].

[3] M. Beccaria and A.A. Tseytlin, Conformal a-anomaly of some non-unitary $6 d$ superconformal theories, JHEP 09 (2015) 017 [arXiv: 1506.08727] [INSPIRE].

[4] I.L. Buchbinder, N.G. Pletnev and A.A. Tseytlin, "Induced" $N=4$ conformal supergravity, Phys. Lett. B 717 (2012) 274 [arXiv:1209.0416] [InSPIRE].

[5] D. Butter, F. Ciceri, B. de Wit and B. Sahoo, All $N=4$ Conformal Supergravities, Phys. Rev. Lett. 118 (2017) 081602 [arXiv: 1609.09083] [INSPIRE]. 
[6] F. Bastianelli, S. Frolov and A.A. Tseytlin, Conformal anomaly of (2,0) tensor multiplet in six-dimensions and AdS/CFT correspondence, JHEP 02 (2000) 013 [hep-th/0001041] [INSPIRE].

[7] M. Kulaxizi and A. Parnachev, Supersymmetry Constraints in Holographic Gravities, Phys. Rev. D 82 (2010) 066001 [arXiv: 0912.4244] [INSPIRE].

[8] M. Beccaria and A.A. Tseytlin, Conformal anomaly c-coefficients of superconformal $6 d$ theories, JHEP 01 (2016) 001 [arXiv:1510.02685] [INSPIRE].

[9] D. Butter, S.M. Kuzenko, J. Novak and S. Theisen, Invariants for minimal conformal supergravity in six dimensions, JHEP 12 (2016) 072 [arXiv:1606.02921] [INSPIRE].

[10] D. Butter, N=1 Conformal Superspace in Four Dimensions, Annals Phys. 325 (2010) 1026 [arXiv:0906.4399] [INSPIRE].

[11] D. Butter, N=2 Conformal Superspace in Four Dimensions, JHEP 10 (2011) 030 [arXiv:1103.5914] [INSPIRE].

[12] D. Butter, S.M. Kuzenko, J. Novak and G. Tartaglino-Mazzucchelli, Conformal supergravity in three dimensions: New off-shell formulation, JHEP 09 (2013) 072 [arXiv:1305.3132] [INSPIRE].

[13] D. Butter, S.M. Kuzenko, J. Novak and G. Tartaglino-Mazzucchelli, Conformal supergravity in five dimensions: New approach and applications, JHEP 02 (2015) 111 [arXiv:1410.8682] [INSPIRE].

[14] J. Wess and B. Zumino, Superspace Formulation of Supergravity, Phys. Lett. B 66 (1977) 361 [INSPIRE].

[15] R. Grimm, J. Wess and B. Zumino, Consistency Checks on the Superspace Formulation of Supergravity, Phys. Lett. B 73 (1978) 415 [INSPIRE].

[16] W. Siegel, Solution to Constraints in Wess-Zumino Supergravity Formalism, Nucl. Phys. B 142 (1978) 301 [INSPIRE].

[17] W. Siegel and S.J. Gates, Jr., Superfield Supergravity, Nucl. Phys. B 147 (1979) 77 [INSPIRE].

[18] P.S. Howe and R.W. Tucker, Scale Invariance in Superspace, Phys. Lett. B 80 (1978) 138 [INSPIRE].

[19] R. Grimm, Solution of the Bianchi identities in SU(2) extended superspace with constraints, in Unification of the Fundamental Particle Interactions, S. Ferrara, J. Ellis and P. van Nieuwenhuizen eds., Plenum Press, New York U.S.A. (1980), pp. 509-523.

[20] P.S. Howe, A superspace approach to extended conformal supergravity, Phys. Lett. B 100 (1981) 389 [INSPIRE].

[21] P.S. Howe, Supergravity in Superspace, Nucl. Phys. B 199 (1982) 309 [InSPIRE].

[22] S.M. Kuzenko, U. Lindström, M. Roček and G. Tartaglino-Mazzucchelli, $4 D N=2$ Supergravity and Projective Superspace, JHEP 09 (2008) 051 [arXiv:0805.4683] [InSPIRE].

[23] P.S. Howe, J.M. Izquierdo, G. Papadopoulos and P.K. Townsend, New supergravities with central charges and Killing spinors in (2+1)-dimensions, Nucl. Phys. B 467 (1996) 183 [hep-th/9505032] [INSPIRE].

[24] S.M. Kuzenko, U. Lindström and G. Tartaglino-Mazzucchelli, Off-shell supergravity-matter couplings in three dimensions, JHEP 03 (2011) 120 [arXiv:1101.4013] [INSPIRE]. 
[25] S.M. Kuzenko and G. Tartaglino-Mazzucchelli, Super-Weyl invariance in $5 D$ supergravity, JHEP 04 (2008) 032 [arXiv: 0802.3953] [INSPIRE].

[26] W.D. Linch, III and G. Tartaglino-Mazzucchelli, Six-dimensional Supergravity and Projective Superfields, JHEP 08 (2012) 075 [arXiv: 1204.4195] [INSPIRE].

[27] D. Butter, S.M. Kuzenko, J. Novak and G. Tartaglino-Mazzucchelli, Conformal supergravity in three dimensions: Off-shell actions, JHEP 10 (2013) 073 [arXiv:1306.1205] [INSPIRE].

[28] D. Butter, B. de Wit, S.M. Kuzenko and I. Lodato, New higher-derivative invariants in $N=2$ supergravity and the Gauss-Bonnet term, JHEP 12 (2013) 062 [arXiv:1307.6546] [INSPIRE].

[29] D. Butter, B. de Wit and I. Lodato, Non-renormalization theorems and $N=2$ supersymmetric backgrounds, JHEP 03 (2014) 131 [arXiv:1401.6591] [INSPIRE].

[30] E. Bergshoeff, E. Sezgin and A. Van Proeyen, Superconformal Tensor Calculus and Matter Couplings in Six-dimensions, Nucl. Phys. B 264 (1986) 653 [Erratum ibid. B 598 (2001) 667] [INSPIRE].

[31] M. Kaku, P.K. Townsend and P. van Nieuwenhuizen, Properties of Conformal Supergravity, Phys. Rev. D 17 (1978) 3179 [INSPIRE].

[32] B. de Wit, J.W. van Holten and A. Van Proeyen, Transformation Rules of $N=2$ Supergravity Multiplets, Nucl. Phys. B 167 (1980) 186 [INSPIRE].

[33] D.Z. Freedman and A. Van Proeyen, Supergravity, Cambridge University Press, Cambridge U.K. (2012).

[34] E. Bergshoeff, A. Salam and E. Sezgin, A Supersymmetric $R^{2}$ Action in Six-dimensions and Torsion, Phys. Lett. B 173 (1986) 73 [INSPIRE].

[35] E. Bergshoeff, A. Salam and E. Sezgin, Supersymmetric $R^{2}$ Actions, Conformal Invariance and Lorentz Chern-Simons Term in Six-dimensions and Ten-dimensions, Nucl. Phys. B 279 (1987) 659 [INSPIRE].

[36] E. Bergshoeff and M. Rakowski, An Off-shell Superspace R(2) Action in Six-dimensions, Phys. Lett. B 191 (1987) 399 [INSPIRE].

[37] F. Coomans and A. Van Proeyen, Off-shell $N=(1,0), D=6$ supergravity from superconformal methods, JHEP 02 (2011) 049 [Erratum ibid. 01 (2012) 119] [arXiv: 1101.2403] [INSPIRE].

[38] E. Bergshoeff, F. Coomans, E. Sezgin and A. Van Proeyen, Higher Derivative Extension of 6D Chiral Gauged Supergravity, JHEP 07 (2012) 011 [arXiv:1203.2975] [INSPIRE].

[39] E.A. Ivanov, A.V. Smilga and B.M. Zupnik, Renormalizable supersymmetric gauge theory in six dimensions, Nucl. Phys. B 726 (2005) 131 [hep-th/0505082] [INSPIRE].

[40] M. Henningson and K. Skenderis, The Holographic Weyl anomaly, JHEP 07 (1998) 023 [hep-th/9806087] [INSPIRE].

[41] L. Baulieu, M.P. Bellon and R. Grimm, BRS Symmetry of Supergravity in Superspace and Its Projection to Component Formalism, Nucl. Phys. B 294 (1987) 279 [inSPIRE].

[42] P. Binetruy, G. Girardi and R. Grimm, Supergravity couplings: A Geometric formulation, Phys. Rept. 343 (2001) 255 [hep-th/0005225] [INSPIRE].

[43] F. Brandt, Counterpart of the Weyl tensor for Rarita-Schwinger type fields, Phys. Lett. B 767 (2017) 347 [arXiv: 1612.03532] [INSPIRE]. 
[44] C. Arias, W.D. Linch, III and A.K. Ridgway, Superforms in six-dimensional superspace, JHEP 05 (2016) 016 [arXiv: 1402.4823] [INSPIRE].

[45] S.M. Kuzenko, J. Novak and I.B. Samsonov, The anomalous current multiplet in $6 D$ minimal supersymmetry, JHEP 02 (2016) 132 [arXiv:1511.06582] [INSPIRE].

[46] S.J. Gates, Jr., Ectoplasm has no topology: the prelude, in Supersymmetries and Quantum Symmetries, J. Wess and E.A. Ivanov eds., Springer, Berlin (1999), p. 46 [hep-th/9709104] [INSPIRE].

[47] S.J. Gates, Jr., Ectoplasm has no topology, Nucl. Phys. B 541 (1999) 615 [hep-th/9809056] [INSPIRE].

[48] S.J. Gates, Jr., M.T. Grisaru, M.E. Knutt-Wehlau and W. Siegel, Component actions from curved superspace: Normal coordinates and ectoplasm, Phys. Lett. B 421 (1998) 203 [hep-th/9711151] [INSPIRE].

[49] L. Castellani, R. D'Auria and P. Fre, Supergravity and superstrings: a geometric perspective. Volume 2: Supergravity, World Scientific, Singapore (1991), pp. 680-684.

[50] G. Girardi and R. Grimm, N=1 supergravity: Topological classes and superspace geometry in four-dimensions, Phys. Lett. B 260 (1991) 365 [InSPIRE].

[51] M.F. Hasler, The Three form multiplet in $N=2$ superspace, Eur. Phys. J. C 1 (1998) 729 [hep-th/9606076] [INSPIRE].

[52] K. Peeters, A Field-theory motivated approach to symbolic computer algebra, Comput. Phys. Commun. 176 (2007) 550 [cs/0608005] [INSPIRE].

[53] K. Peeters, Introducing Cadabra: a symbolic computer algebra system for field theory problems, hep-th/0701238 [INSPIRE].

[54] W. Siegel, Superfields in Higher Dimensional Space-time, Phys. Lett. B 80 (1979) 220 [INSPIRE].

[55] E. Bergshoeff, E. Sezgin and A. Van Proeyen, (2,0) tensor multiplets and conformal supergravity in D =6, Class. Quant. Grav. 16 (1999) 3193 [hep-th/9904085] [INSPIRE].

[56] H. Lü, Y. Pang and C.N. Pope, Conformal Gravity and Extensions of Critical Gravity, Phys. Rev. D 84 (2011) 064001 [arXiv: 1106.4657] [INSPIRE].

[57] Y. Pang, One-Loop Divergences in 6D Conformal Gravity, Phys. Rev. D 86 (2012) 084039 [arXiv: 1208.0877] [INSPIRE].

[58] H. Lü, Y. Pang and C.N. Pope, Black Holes in Six-dimensional Conformal Gravity, Phys. Rev. D 87 (2013) 104013 [arXiv:1301.7083] [INSPIRE].

[59] P.S. Howe, G. Sierra and P.K. Townsend, Supersymmetry in Six-Dimensions, Nucl. Phys. B 221 (1983) 331 [INSPIRE].

[60] J. Koller, A six-dimensional superspace approach to extended superfields, Nucl. Phys. B 222 (1983) 319 [INSPIRE].

[61] M. Ozkan and Y. Pang, All off-shell $R^{2}$ invariants in five dimensional $\mathcal{N}=2$ supergravity, JHEP 08 (2013) 042 [arXiv: 1306.1540] [InSPIRE].

[62] E. Sokatchev, Off-shell Six-dimensional Supergravity in Harmonic Superspace, Class. Quant. Grav. 5 (1988) 1459 [INSPIRE]. 
[63] M. Ozkan, Supersymmetric curvature squared invariants in five and six dimensions, Ph.D. Thesis, Texas A\&M University (2013) [http://oaktrust.library.tamu.edu/bitstream/handle/ 1969.1/151223/OZKAN-DISSERTATION-2013.pdf?sequence=1].

[64] J. Grundberg and U. Lindström, Actions for Linear Multiplets in Six-dimensions, Class. Quant. Grav. 2 (1985) L33 [INSPIRE].

[65] H. Nishino and S.J. Gates, Jr., Manifestly supersymmetric extensions of (curvature) ${ }^{2}$-terms in six-dimensional $N=2$ supergravity, Phys. Lett. B 173 (1986) 417 [INSPIRE].

[66] S.J. Gates, Jr., Superconformal Transformations and Six-dimensional Space-time, Nucl. Phys. B 162 (1980) 79 [INSPIRE].

[67] B.E.W. Nilsson, Superspace Action for a Six-dimensional Nonextended Supersymmetric Yang-Mills Theory, Nucl. Phys. B 174 (1980) 335 [InSPIRE].

[68] J. Wess, Supersymmetric gauge theories, in proceedings of the 5th Johns Hopkins Workshop on Current Problems in Particle Theory: Unified Field Theories and Beyond, Baltimore, Maryland, 25-27 May 1981.

[69] P.S. Howe, G. Sierra and P.K. Townsend, Supersymmetry in Six-Dimensions, Nucl. Phys. B 221 (1983) 331 [INSPIRE].

[70] S.J. Gates, Jr. and W. Siegel, Understanding Constraints in Superspace Formulations of Supergravity, Nucl. Phys. B 163 (1980) 519 [INSPIRE].

[71] M. Awada, P.K. Townsend and G. Sierra, Six-dimensional Simple and Extended Chiral Supergravity in Superspace, Class. Quant. Grav. 2 (1985) L85 [INSPIRE]. 